\title{
Development of environmental health and safety database for small communities
}

\author{
Ragava S. Nadig \\ West Virginia University
}

Follow this and additional works at: https://researchrepository.wvu.edu/etd

\section{Recommended Citation}

Nadig, Ragava S., "Development of environmental health and safety database for small communities" (2001). Graduate Theses, Dissertations, and Problem Reports. 1203.

https://researchrepository.wvu.edu/etd/1203

This Thesis is protected by copyright and/or related rights. It has been brought to you by the The Research Repository @ WVU with permission from the rights-holder(s). You are free to use this Thesis in any way that is permitted by the copyright and related rights legislation that applies to your use. For other uses you must obtain permission from the rights-holder(s) directly, unless additional rights are indicated by a Creative Commons license in the record and/ or on the work itself. This Thesis has been accepted for inclusion in WVU Graduate Theses, Dissertations, and Problem Reports collection by an authorized administrator of The Research Repository @ WVU. For more information, please contact researchrepository@mail.wvu.edu. 


\title{
Development of Environmental Health and Safety Database for Small Communities
}

\author{
Ragava Nadig
}

Thesis submitted to

The College of Engineering and Mineral Resources

at West Virginia University in partial fulfillment of the requirements for the degree of

\section{Master of Science \\ in \\ Industrial Engineering}

\author{
Rashpal S. Ahluwalia, Chair \\ David Whaley \\ Sanjay Saxena
}

Department of Industrial and Management Systems Engineering,

Morgantown, West Virginia.

2001

Keywords: Process Analysis Technique, Waste Water, Drinking Water, Solid Waste, Small Communities 


\section{Abstract}

\section{Development of Environmental Health and Safety Database for Small Communities}

\section{Ragava Nadig}

The National Environmental Services Center (NESC) is a group of programs, national in scope, that provide a variety of environmental services directly to small community officials, engineers, technicians, regulatory officials, and private citizens.

The primary mission of each of the NESC programs is to protect public health and the environment of America's small and rural communities-those communities of less than 10,000 people. The NESC programs provide information, technical assistance, and training to help small communities deal with their wastewater, drinking water, and solid waste treatment issues.

Thousands of informational materials pass through the organization each year. This material ranges from manufacturing product literature to design guidelines to state regulations to the most current field research to educational products for the general public. In order to keep on top of all this information, the organization maintains several databases.

The objective of this project was to research NESC needs and develop an effective and efficient informational product review process which will help in reducing process time, better information storage and retrieval, etc. The work also included developing a products database for storage and a corresponding search engine for easy retrieval of information.

Environmental health and safety database, also known in NESC as "Products Database", represents environmental information materials about waste water, drinking water and solid waste. 


\section{ACKNOWLEDGEMENT}

It is my pleasure to acknowledge everyone who has been involved directly or indirectly during the course of my masters program.

I would like to thank my parents and my brother for their constant support in helping me complete my master's degree. I am very grateful to Dr. Rashpal Ahluwalia for helping me during the course of my studies at West Virginia University. His suggestions and comments have been invaluable. I would like to thank my committee members Dr. David Whaley and Mr. Sanjay Saxena for their constant support throughout this project. I would like to thank NESC (The National Environmental Services Center), whose support and encouragement made this project possible. I would like to thank Mr. Vernon Deal and Ms. Jeanne Allen for their support, time and complete cooperation throughout this project.

I am very grateful to Mr. Wiselin Dhas Mathuram for helping me with the database problems. I would like to thank Mr. Shivananda M.Purnachandra, Mr. Praveen Neogi, Mr. Vineet Shastri and Mr. Niranjan Mysore with whom I stayed in Morgantown, for their constant support and valuable advice. 


\section{TABLE OF CONTENTS}

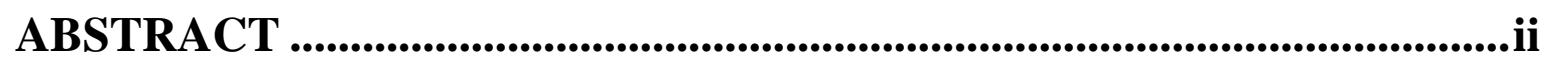

ACKNOWLEDGEMENT .....................................................................

TABLE OF CONTENTS .........................................................................

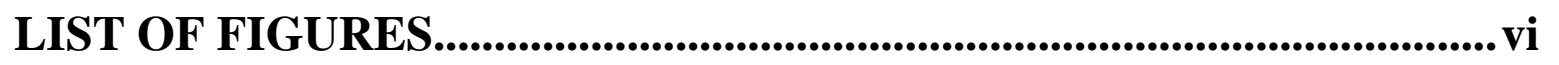

CHAPTER 1 INTRODUCTION ............................................................

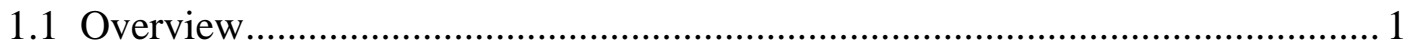

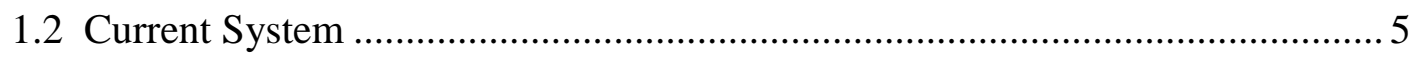

1.2.1 Product Review Process ....................................................................... 5

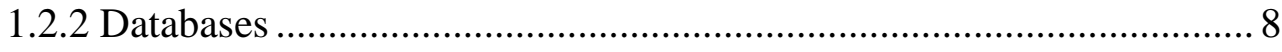

CHAPTER 2 PROBLEM STATEMENT ............................................... 11

2.1 System Limitations ……………………………............................................. 11

2.1.1 Identification of new items .......................................................... 11

2.1.2 Evaluation of new items .................................................................. 11

2.1.3 Inclusion of new item in the products database ................................... 11

2.1.4 Promotions ....................................................................................... 11

2.1.5 Review of the products database ……………………………........ 11

2.1.6 Information retrieval..................................................................... 12

CHAPTER 3 SOLUTION APPROACH ................................................ 13

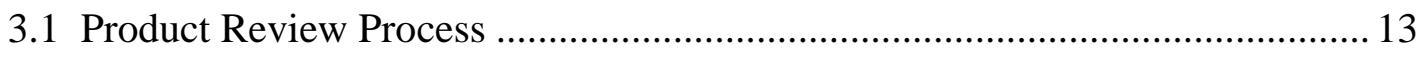

3.1.1 Process Management ....................................................................... 13

3.1.2 Process Analysis ............................................................................... 14

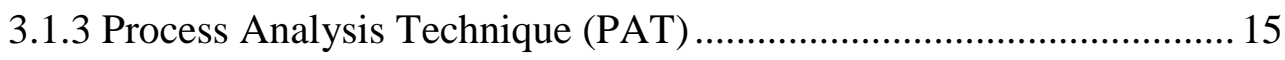

3.1.3.1 Management decision to have process analysis performed .15

3.1.3.2 Define limits of the process ................................................ 15

3.1.3.3 Prepare and issue an action plan document ........................ 16 


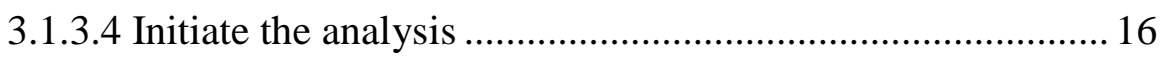

3.1.3.5 Hold an information meeting ............................................... 16

3.1.3.6 Conduct process interviews ................................................. 16

3.1.3.7 Analyze and revise the process ............................................ 17

3.1.3.8 Present the revised process ................................................. 17

3.1.3.9 Establish an implementation strategy ................................... 17

3.1.4 Proposed Process ........................................................................ 18

3.1.4.1 Identification/Initial review of item ……………................. 18

3.1.4.2 Technical/Promotional review of item ................................. 18

3.1.4.3 Inclusion/Periodic review of item......................................... 19

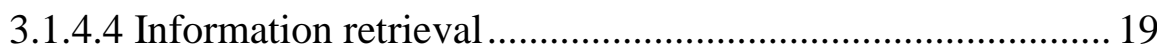

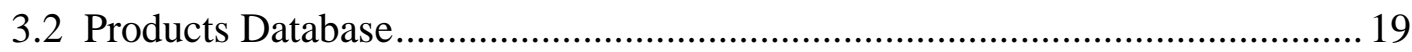

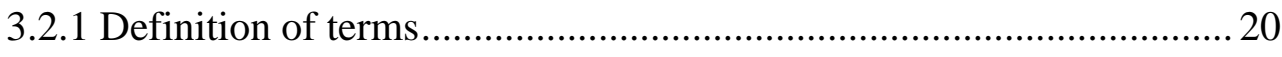

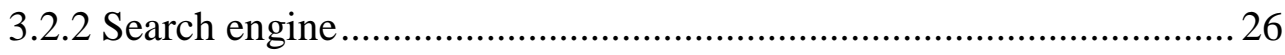

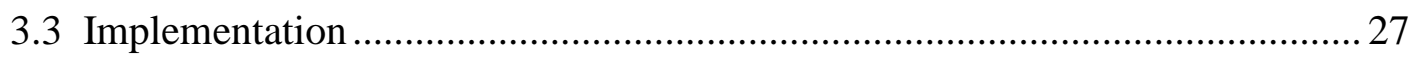

CHAPTER 4 CONCLUSIONS/CONTRIBUTIONS...............................66

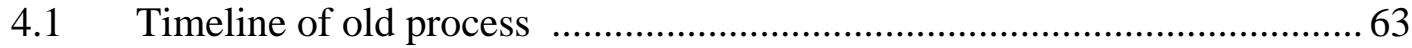

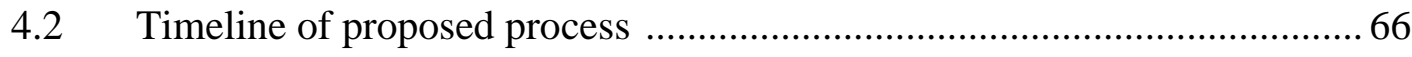

4.3 Advantages of proposed process over old process ...................................... 67

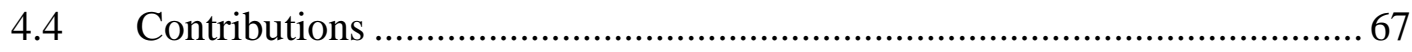

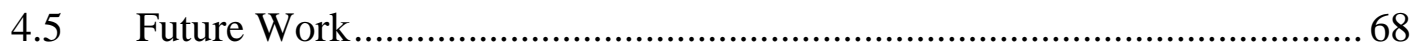

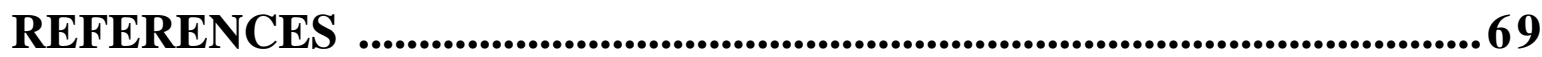

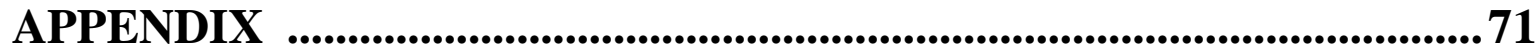




\section{LIST OF FIGURES}

Figure 3.1: Products Database Front End Screen ................................................ 21

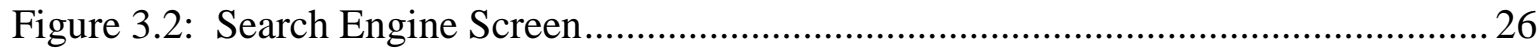

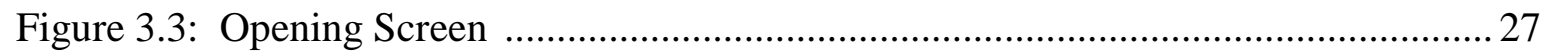

Figure 3.4: Screen showing the Run Button ..................................................... 28

Figure 3.5: Main Front End Screen …................................................................ 29

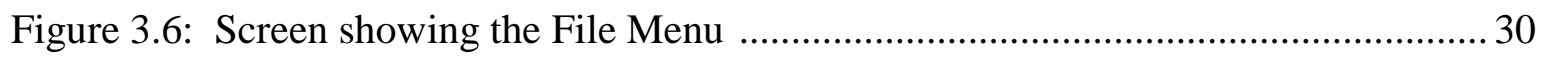

Figure 3.7: Create New Database window ........................................................ 31

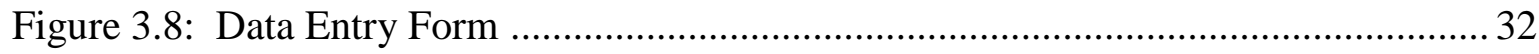

Figure 3.9: Screen showing the Basic information of an item ..................................... 33

Figure 3.10: Technical Review Questionnaire window ......................................... 34

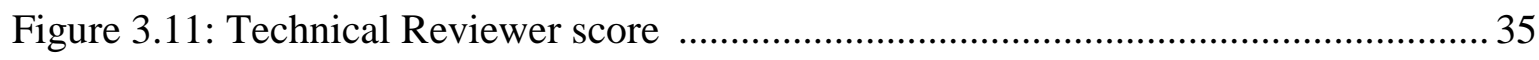

Figure 3.12: Window for Second Technical Reviewer ............................................ 36

Figure 3.13: Technical Review Result window .................................................... 37

Figure 3.14: Item Number Entry Screen ............................................................ 38

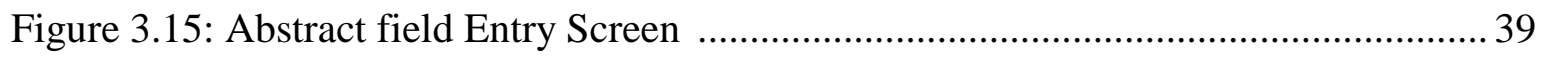

Figure 3.16: Keyword Edit Click Window ........................................................ 40

Figure 3.17: Main Keyword Window ........................................................................ 41

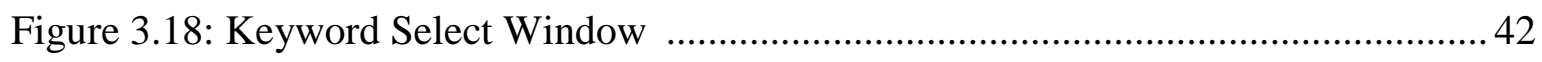

Figure 3.19: Selected Keyword Display Screen .................................................... 43

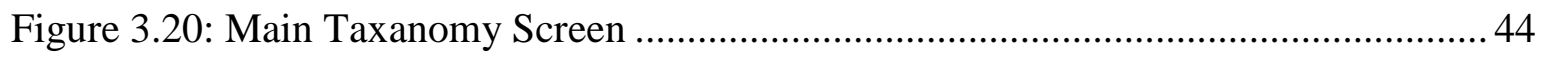

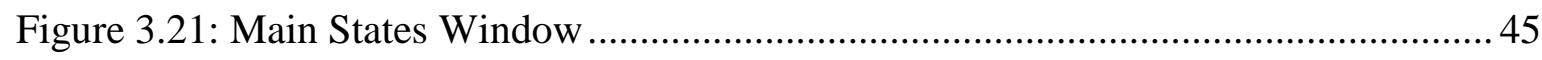




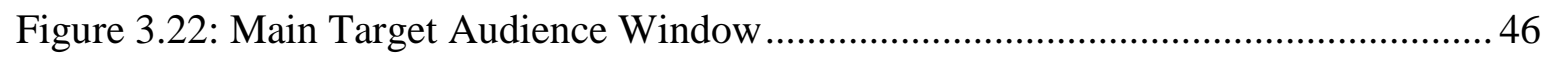

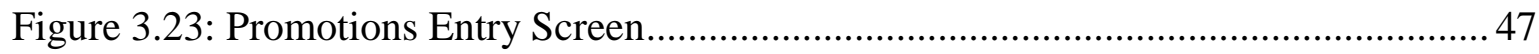

Figure 3.24: First Record in the Database ………………......................................... 48

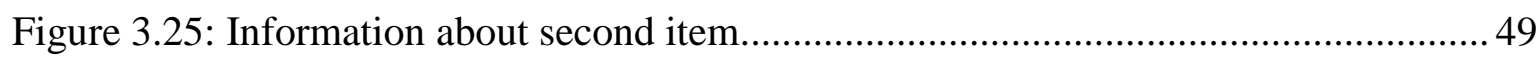

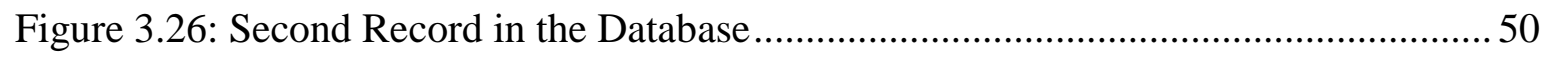

Figure 3.27: Information about one more record.............................................................51

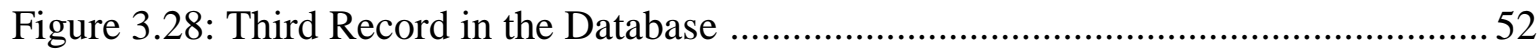

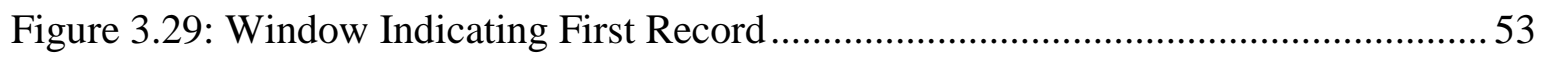

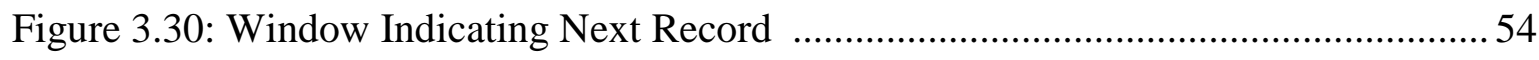

Figure 3.31: Window Indicating Previous Record …………………............................54

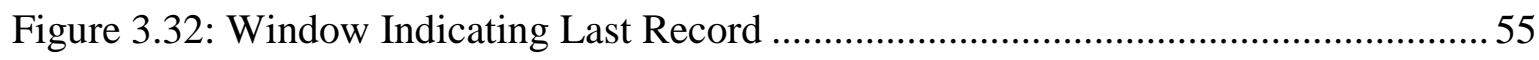

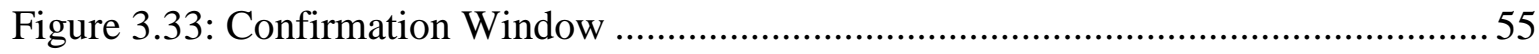

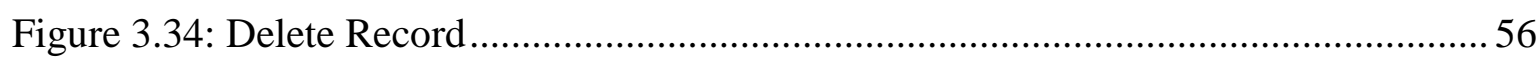

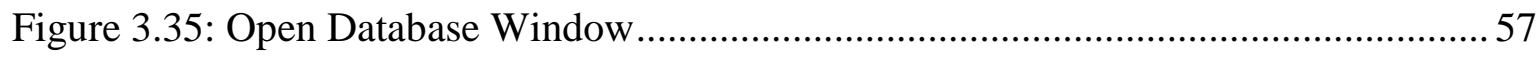

Figure 3.36: Record one of Products Database ……………….......................................5 58

Figure 3.37: Record Two of Products Database ...............................................................5 58

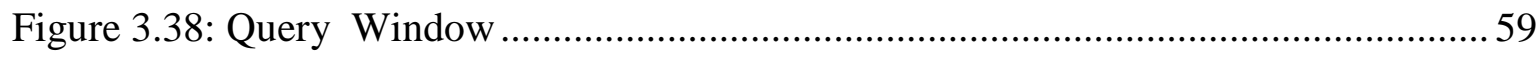

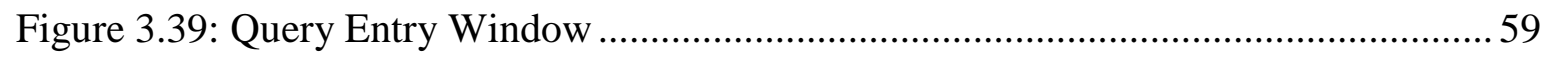

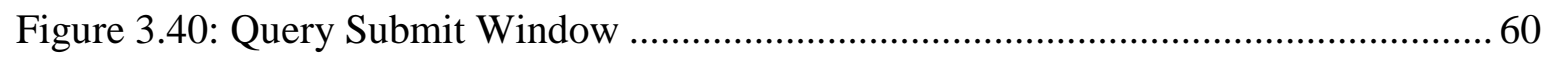

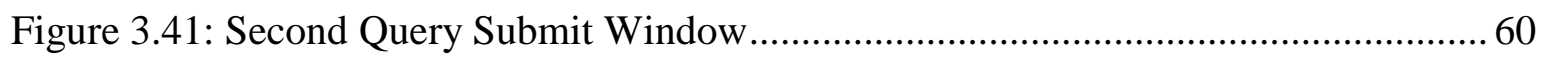

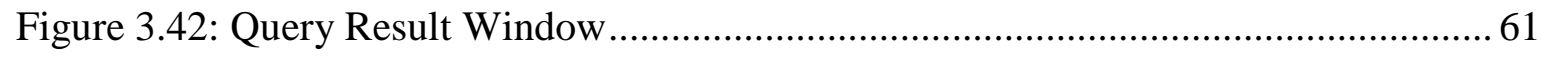

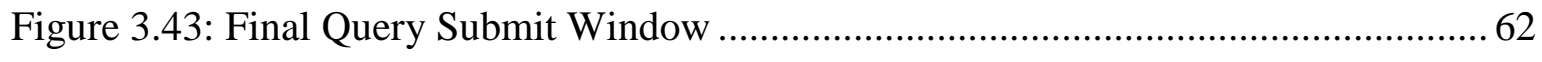

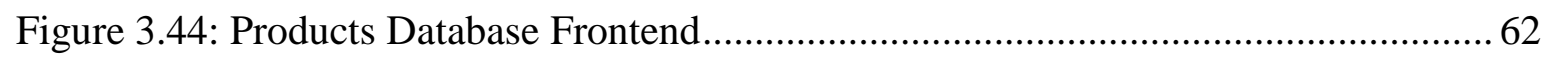




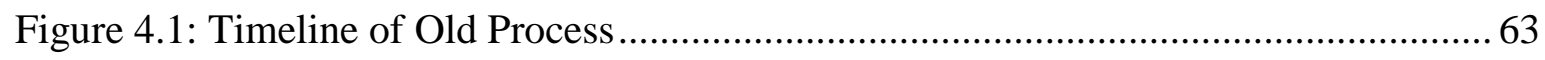

Figure 4.2: Timeline of Proposed Process ............................................................... 65 


\section{CHAPTER 1: INTRODUCTION}

This project was developed to recommend procedures to streamline the turnaround time for technical screening and approval of information products and database management of these products for "The National Environmental Services Center"(NESC), organization within "The National Research Center for Coal and Energy"(NRCCE).

In collaboration with the faculty of West Virginia University, the National Research Center for Coal and Energy (NRCCE) identifies, develops, promotes, coordinates, supports, and conducts multidisciplinary energy and environmental research and service programs that are important to industry, the state, and the nation. The NRCCE accomplishes its mission through the efforts of faculty, professionals, students, and external collaborators working through organized program units, each of which serves the specialized needs of its sponsor and user community in discovering new knowledge; applying, extending, and generating new technologies; transferring information through multimedia systems and learning programs; and by providing support services to facilitate developing new programs, conducting and completing research and service activities, and transferring knowledge [13].

\subsection{OVERVIEW OF WVU NRCCE DIVISIONS AND PROGRAMS}

\subsubsection{ALTERNATIVE FUELS}

\subsubsection{National Alternative Fuels Training Program Consortium}

The NAFTPC is a consortium of educational institutions working with fuel providers; equipment and parts manufacturers; federal and state agencies; industry; and professional, educational, and training associations [14]. 


\subsubsection{Marine Engine Emissions Research Program}

The Marine Engine Emissions Research Program was funded in FY96 by the U.S. EPA and involves analysis of the impact on our nation's waterways of petroleum-based fuels from both commercial and recreational marine uses.

\subsubsection{MARKET ENHANCEMENT \& PROGRAM DEVELOPMENT}

The Market Enhancement and Program Development Division funds "seed" projects_-

research projects proposed by WVU faculty that the Center deems promising for stimulating economic development and for attracting industry and/or government support on a large scale, providing an opportunity to demonstrate the strategic importance of these projects on a small scale.

\subsubsection{Environmental Technology Division}

The Environmental Technology Division includes the National Mine Land Reclamation Center, the Environmental Restoration and Hazardous Waste Management Program, and the West Virginia Water Research Institute. In addition, the Division has been instrumental in the start-up of a number of environmental remediation programs including the Acid Drainage Technology Initiative, the Appalachian Clean Streams Initiative, the National Environmental Education Training Center, and the Mid-Atlantic Highlands Coordinating Council. The Division is also headquarters for the West Virginia Water Resources Research Institute and the Emissions Control By-Products Consortium.

\subsubsection{RESOURCE EXTRACTION}

\subsubsection{Appalachian Oil and Natural Gas Research Consortium(AONGRC)}

A partnership among the WVU Departments of Geology and Geography, and Petroleum and Natural Gas Engineering, as well as four state geological surveys, the AONGRC seeks to increase the environmentally acceptable and economically viable extraction of petroleum resources in the Appalachian Basin. 


\subsubsection{TECHNICAL COMMUNICATIONS}

Within the Technical Communications Division, the WVU NRCCE's teleconferencing facilities became fully operational during FY96. The Center broadcasts live via public access TV and satellite, as a WVU tribute to Senator Robert C. Byrd and provides a national teleconference workshop located for the National Alternative Fuels Training Center.

\subsubsection{NATIONAL ENVIRONMENTAL SERVICES CENTER DIVISION}

The WVU NRCCE is home to the National Environmental Services Center ( NESC). The NESC is a group of programs, national in scope, that provide a variety of environmental services directly to small community officials, engineers, technicians, regulatory officials, and private citizens [15]. The NESC includes four programs:

- The National Small Flows Clearinghouse (NSFC)

- The National Onsite Demonstration Program (NOPD)

- The National Drinking Water Clearinghouse (NDWC)

- The National Environmental Training Center for Small Communities (NETCSC).

The primary mission of each of the NESC programs is to protect public health and the environment of America's small and rural communities-those communities of less than 10,000 people. Collectively, the NESC programs provide information, technical assistance, and training to help small communities deal with their wastewater, drinking water, and solid waste treatment issues.

\subsubsection{The National Small Flows Clearinghouse}

The NSFC's mission is to help small communities effectively deal with their wastewater treatment needs [16]. More than 25 percent of American homes use onsite wastewater treatment systems, such as septic tank systems, to treat their wastewater. The NSFC, established in 1979 and funded through the U.S. EPA, is the only national resource of its type dealing with small community wastewater infrastructure. 
All NSFC services are available free or at a minimal charge. These include two free newsletters (Small Flows Quarterly and Pipeline); a toll-free technical assistance hotline; a World Wide Web site (http://nsfc.wvu.edu); comprehensive databases containing information on wastewater literature, regulations, products, facilities, manufacturers, contact \& referrals, and consultants; and a vast inventory of technical and educational products.

The NSFC provides free assistance to an average of 30,000 telephone callers per year. It distributes about 110,000 low-cost educational products annually. Collectively, subscribers and one-time information requesters to NSFC publications number over 70,000.

\subsubsection{The National Onsite Demonstration Project}

The NODP is an outgrowth of the NSFC that is conducting alternative wastewater treatment demonstrations throughout the nation [19]. Funded through a cooperative agreement between the U.S. EPA and the NSFC, the purpose of these projects is to design, construct, implement, and demonstrate alternative onsite wastewater treatment technologies. Demonstration projects in over 15 states aid community acceptance and provide opportunities

for research. NODP projects have helped alleviate wastewater treatment problems, and constructed demonstration facilities to educate the public about alternative wastewater treatment technologies. The information and data gathered from these projects help other small communities in similar situations make environmentally sound choices about their wastewater treatment methods.

\subsubsection{The National Drinking Water Clearinghouse}

Established in 1991, the NDWC assists small communities by collecting, developing, and providing timely information relevant to drinking water issues [17]. It is funded through a technical assistance and training grant from the U.S. Department of Agriculture's Rural Utilities Service (RUS) and is an extension of the USDA's commitment to provide technical assistance to America's rural water facilities. 
The NDWC maintains a World Wide Web site (www.ndwc.wvu.edu) and several databases on drinking water technologies, regulations, and related issues. NDWC technical staff provide technical assistance to drinking water system operators, community officials, and the public. The NDWC's educational videotapes, manuals, and booklets assist operators, regulators, educators, extension agents, the media, and the general public. On Tap, the NDWC magazine reaches over 20,000 readers every quarter which include: over 4,000 managers, consultants, regulators, and water system operators.

\subsubsection{The National Environmental Training Center for Small Communities}

NETCSC improves the public health and environment of small communities by providing training and training-related information and referral services in the areas of wastewater, drinking water, and solid waste [18]. This U.S. EPA-supported program develops and disseminates environmental training programs; promotes partnerships among environmental trainers nationwide; and serves as a national resource center for environmental trainers. NETCSC has delivered over 60 training courses to over 1,500 participants and has developed 28 comprehensive programs for environmental trainers. NETCSC provides both skills- and knowledge-based training resources to environmental training professionals and maintains several databases, including the most comprehensive listing of training activities in the nation, as well as training modules, curricula, and other materials available nationwide. $E$ train, the NETCSC newsletter, is distributed quarterly to nearly 6,500 subscribers and is available through the program's Web site at www.netc.wvu.edu.

\subsection{CURRENT SYSTEM}

\subsubsection{Product Review Process}

The Process for identifying an informational product, initially known within NESC as an "item"; until it's been promoted in the Catalogs, Newsletters, etc., can be divided into 6 stages.

- Identification of new items.

- Evaluation of new items.

- Inclusion of new items in the database. 
- Promotions.

- Review of the database.

- Information retrieval.

\subsubsection{IDENTIFICATION OF NEW ITEMS}

First, the item is obtained through various sources, which include but are not limited to the following: The supervisor actively seeks out new "Potential Products"; employees hear of a new item and request that the supervisor obtain a copy for review; employees come across an item and pass it on to the supervisor to send it through the process; external people send a copy of a document/material to be considered as a new product.

This material ranges from design guidelines to state regulations to the most current field research to educational products for the general public.

\subsubsection{EVALUATION OF NEW ITEMS}

The item ( potential new product) is given to the secretary to log into the product database. The secretary searches the database to find out if the item is already in the database. If the item is not present, the item is logged on into the database. This includes assigning a Preliminary Accession Number known within NESC as a product number ( $\mathrm{P} \#$ ) and all the basic information ( title, author, medium, etc.. ). All the items that enter the review process is given a product number. Item is then passed to the technical assistants for technical review. Technical Assistants review the material and completes the information on Information Tracking Screen known within NESC as a buck sheet. A buck sheet is just a plain sheet of paper. This includes providing an abstract, keywords, taxonomy codes, etc. The technical assistants determine if the item should be offered as a product based on whether the information: is up to date, meets the needs of the customer, etc. Based on these criteria, technical assistants either accept or reject the item being a product. If the item is accepted, the technical assistants assign a letter code designating the NESC program applicable and then passes the item with the buck sheet back to the secretary for completion of data entry. If an item is rejected this is indicated, and the item is sent back to the secretary. 


\subsubsection{INCLUSION OF NEW ITEMS INTO THE DATABASE}

Once the secretary receives the item with the completed buck sheet, all the information that is in the buck sheet is manually entered into the database. This is the final stage of decision making to whether offer an item as a product or not. This is because some companies will not allow distributing their products; others may require that the material be purchased; and few others may not allow for photo-copying the document. The secretary contacts the owner of the item to find out if NESC can use and promote their item as a product. Once this is resolved, the secretary meets with the supervisor to begin the process of obtaining bulk copies. If a Product is accepted or rejected, it is marked as such and the secretary files the document in a single file of original items and their buck sheets files in the technical assistance file room, failing to segregate accepted from rejected items.

\subsubsection{PROMOTIONS}

After a product has been accepted \& copies obtained for distribution, a copy of the product along with the buck sheet is sent to the promotions specialist for a promotional abstract to be written. This abstract is used in all publication, including the product catalog, where a product is being promoted.

\subsubsection{PERIODIC REVIEW OF THE PRODUCTS DATABASE}

This stage is to find out when an item has to be re-evaluated so that the information about an item is not outdated, or the information contained in the product is no longer relevant etc. This stage of the process is currently not implemented by all programs within NESC.

\subsubsection{INFORMATION RETRIEVAL:}

Once all the information about an item is stored in the database, it is used for assistance on various topics. Several hundred requests for assistance are received each month. When requests for assistance are received, staff conducts searches of the "Products Database" ( along with searches of other databases ) to match products and other materials to customer needs. 


\subsubsection{Databases}

Thousands of informational materials pass through the organization each year. This material ranges from manufacturing product literature to design guidelines to state regulations to the most current field research to educational products for the general public. In order to keep on top of all this information, the organization maintains several databases.

\subsubsection{Bibliographic Databases}

The Bibliographic Databases are a collection of thousands of articles dealing with onsite and small community, drinking water and waste water related topics. The articles are collected from more than 90 journals and magazines, as well as conference proceedings and other research papers. The database is continually growing, as journals are reviewed and articles are selected. Each article is assigned keywords and an abstract is written, reviewed, and then entered into the database system. NSFC and NDWC use this database.

\subsubsection{Manufacturers and Consultants Database}

The Manufacturers and Consultants Database houses a list of industry contacts for wastewater products and consulting services. This database serves both as a reference for engineers, private citizens, and small community officials and a referral database for wastewater products and trade items. Currently, the database contains entries for more than 1,200 manufacturers and consultants. This database is currently used by NSFC.

\subsubsection{Facilities Database}

The facilities database helps operators, homeowners, engineers, government officials, and wastewater managers obtain information on facilities using conventional, innovative, and alternative wastewater treatment technologies. This database is currently used by NSFC.

\subsubsection{Contacts and Referrals Repository}

The contacts and referrals repository lists organizations involved in onsite and small community, drinking and wastewater infrastructure at the national, state, and local levels. These organizations offer assistance through finance, research, regulations, technical and outreach assistance, and training. This database is used by NSFC and NDWC. 


\subsubsection{Regulations Repository:}

The NSFC maintains an extensive collection of regulations for onsite wastewater treatment systems. The regulations repository contains onsite wastewater regulations from 48 out of 50 states, additional regulatory products from other state and national regulatory entities, such as EPA; and items prepared by the NSFC staff.

\subsubsection{RESULTS Database:}

The Registry of Equipment Suppliers of Treatment Technologies for Small Systems, called "RESULTS", is a database that serves as an initial reference point for small communities, regulators, and others seeking information about appropriate drinking water technologies. Currently housing information on more than 100 small community sites around the country, RESULTS is continually being updated and populated with new information. This database is used by NDWC.

\subsubsection{Environmental Information Warehouse (EIW) Database}

This NETCSC database contains all materials possessed in the NETCSC's resource center, each of which will be assigned an EIW number upon receipt and review by NETCSC personnel. EIW is the master database for the following four breakout databases:

- Activities Database: This contains information pertaining to conferences, certification courses, symposia, and other events that accomplish environmental training.

- Materials Database: This contains training materials such as videos, cassette tapes, slides, overheads, software, training needs assessment surveys, references, instructors' and students' guides, course books, maps, course evaluations, laboratory exercises, etc., received by NETCSC.

- Organizations' Database: This contains information about organizations that provide some environmental training or technical assistance to small communities.

- Trainer's Database: This contains information about individuals who deliver environmental training or who develop methods and theories of training, manuals or supporting materials relative to wastewater, solid waste, drinking water, or adult education. 


\subsubsection{Environmental Health and Safety Database}

\section{Environmental Health and Safety Database also known as Products Database}

represents environmental information materials about wastewater, drinking water and solid waste. These materials and resources consists of training curricula, video tapes, software, brochures, fact sheets, books, journals, and various publications dealing with design, finance, management, public education, general information technologies, regulations and research, relevant to small communities. Often, items of interest to specific audiences, or items that cover specific technologies or environmental issues are received by the organization for consideration to be offered as a "Product " and thereby promoted to the customers. The Products Database tracks and houses these materials. This database is shared by the 3 organizations NSFC, NDWC, NETCSC. 


\section{CHAPTER 2: PROBLEM STATEMENT}

\subsection{SYSTEM LIMITATIONS}

\subsubsection{Identification of new item}

In the current process there is no record to indicate when the whole process starts. It is not found out initially if the item can be offered as a product, which would reduce the time required for the whole process.

\subsubsection{Evaluation of new item}

Time taken by this stage is too long because initially the technical assistants enter all the information on a buck sheet and once the buck sheet is passed back to the secretary, the information is entered into the database. Since there is only one person reviewing the items, it leads to long process time and it can also be biased upon his/her decision.

\subsubsection{Inclusion of new item in the Products Database}

At present, when an item is rejected, no information is provided why it was rejected. Data entry capability for technical assistants is not available which will reduce the process time. With the current database the organization is facing platform issues and is not yet able to upgrade the network on to higher versions of MAC-OS.

\subsubsection{Promotions}

The promotional abstract is written at the end of the whole process, which dampens the smooth flow of work. A copy of an item is sent to the promotions specialist for a promotional abstract to be written after the secretary enters all the information into the database and once bulk copies are obtained.

\subsubsection{Periodic Review of the Products Database}

Current database does not consistently include when the item has to be re-reviewed. 


\subsubsection{Information Retrieval}

Currently, searches cannot be done by organizations, products under review, etc. The present system is not user friendly. For example it is often difficult to locate an item in the database even with the title.

In this thesis project, The existing process was studied. The NESC needs has been researched and a new process has been developed which will help in reducing process time, better information storage and retrieval, etc. This work also includes the development of a database, which will house all the information. A corresponding search engine has also been developed. 


\section{CHAPTER 3: SOLUTION APPROACH}

\subsection{Product Review Process}

In this part we define what process, process management and process analysis mean and the kind of technique used to develop the new process. The new process is defined in detail and compared with the old process. The flow chart of the proposed process is also shown.

\subsubsection{Process Management}

A process is a bounded set of interrelated work activities each having prescribed inputs and outputs. It has a well-defined beginning and end. A process is essentially "a method for doing things" [3].

Process Management has evolved from the realization that techniques of managing workflow in manufacturing can also be applied to non-manufacturing operations of a business. Process Management has proven to be an effective way to examine a business process, many operational improvements can be achieved by this approach with relatively little investment in resources.

The fundamentals of process management can be divided into 3 phases:

- Process Initialization

- Process Definition

- Process Control

Process Initialization: In this phase, the ownership and the boundary settings of the process are clearly defined. That is, A process owner is one who is accountable for the functioning and performance of a process and has the authority to make or oversee a change in the process. The second major part is defining the boundaries within which the process is to be defined and managed. 
Process Definition: Defining the process involves providing a means for both understanding and communicating operational details to those involved. It also provides a baseline, or standard, for evaluating improvement. In most cases, merely defining the process can reveal redundant and needless steps and other non-value-adding activities. Thus, definition becomes key to understanding an operation.

Process Control: The final phase of process management is process control. Process control basically consists of 3 steps. Establishing points of control, implementing measurements, and regulating the process by obtaining feedback and performing corrective action.

\subsubsection{Process Analysis}

Process Analysis is a systematic way of defining the activities and tasks within an operation, generally at a departmental or work-group level.

There are several approaches to examining a process for improvement, depending on whether work, materials or information flows are involved. They are:

- Departmental Work Product Analysis(DWPA)

- Departmental Activity Analysis(DAA)

- Department Quality Analysis(DQA)

- Process Analysis Technique (PAT)

Departmental Work Product Analysis: DWPA is based on detailing, classifying, and examining the activities of a group.

Departmental Activity Analysis: DAA was developed to determine the types of activities within a department and to obtain a cost-of-quality estimate.

Department Quality Analysis: DQA groups work product analysis on management rather than employee input. DQA is very similar to DAA. 
For complex organizations that encompass several functional boundaries of an organization, analysis is more extensive and time consuming and requires greater coordination and planning. The Process Analysis Technique is a structured approach that can be used in larger, cross-functional processes.

\subsubsection{Process Analysis Technique (PAT)}

PAT is essentially a step - by - step structured approach to defining the tasks necessary to execute a set of activities and simplifying them. PAT requires the involvement of 5 types of individuals: 1) The process owner, 2) Process analyst, 3) Lead coordinator (having knowledge of the operation to be studied) who serves as support to the analyst, 4) Management associated with the process, and 5) The people working within the process itself $[6]$.

Here, the steps involved in using PAT are explained and the way it has been used for the current project.

\subsubsection{Management Decides to have process analysis performed}

This is the starting point of PAT. In this stage, management decision is sought to go ahead with the analysis. However, it is an explicit requirement in PAT that this decision be made by the process owner and not by some third party members. For this project, NESC as a whole was considered to be the management and the process owner. Also at this stage the person performing the analysis is also identified (known as the Process Analyst). For this project I was the process analyst. By obtaining the necessary permission, we proceed to the second stage.

\subsubsection{Define limits of the process}

In this stage the process owner establishes the process boundaries in order to identify the beginning and end of the process and the sub-processes to be analyzed. This stage also involves identification of departments, work groups and managers involved in the process and also a lead coordinator to support the analyst. For our project, the different departments were identified (NETC, NSFC, NDWC, NODP) and also a lead coordinator was identified in 
Ms. Jeanne Allen. The people who work under these departments form the work groups and the managers correspond to the department managers.

\subsubsection{Prepare and issue action plan}

After the process team is identified and boundaries defined, the process owner prepares a plan of action report which contains information like: the process under consideration, objectives of the analysis, start/end dates for the analysis, statement of current problems and a statement of method and approach to be used. This action plan is also known as a Document of Understanding (DOU).

\subsubsection{Initiate the analysis}

In this stage of the process, the analyst reviews the objectives and approach for analysis and initiates meetings with managers and work groups. Also the department manager identifies the people involved with the process under consideration

\subsubsection{Hold an information meeting}

Once the employees are identified, the analyst holds information meetings at which the process owner, lead coordinator, managers, and task experts attend to discuss the initiation of the analysis. Also the lead coordinator explains the employee participation for the next stage.

\subsubsection{Conduct Process Interviews}

In this stage, the analyst interviews the task experts individually and develops a process flow based on their description of the tasks performed. During each interview, the time taken to perform a task, the kind of support needed to perform a task, the repeatability of the task, actions taken to perform a task, and what can be done to improve the task are noted down. Once all the information is gathered the analyst meets with the lead coordinator to finalize the process flow and is now ready for the analysis phase. 


\subsubsection{Analyze and revise the process}

In this stage the analyst and lead coordinator analyze the assembled set of tasks for improvement. As the analysis develops, the analyst questions all parts of the process and considers alternatives. The analyst distinguishes essential from nonessential tasks, tasks that can be eliminated or combined with others, etc. while working with the lead coordinator. At the end of analysis, a revised process flow diagram is prepared by the analyst with recommendations for improvement.

\subsubsection{Present the revised process}

Once the revised process is completed, the analyst and lead coordinator meets with all the participants. The original and improved process flows are presented, highlighting the changes proposed. The advantages of the improved process are noted down.

\subsubsection{Establish an implementation strategy}

Upon approval of the revised process, the process team develops an implementation strategy.

As seen from the above steps, PAT is not a straightforward, traditional process analysis. It involves various communication steps between employees and analyst, and also consideration of certain social, behavioral, and organizational elements embedded in the process.

\subsubsection{Proposed Process ( PRODUCT REVIEW PROCESS )}

\subsubsection{IDENTIFICATION / INITIAL REVIEW OF ITEMS}

The first stage in the Product Review Process (PRP) is identification/initial review. In this stage, a "Informational Product" known within NESC as an item, which can be a potential product, is obtained through various sources, including but not limited to: supervisor actively seeking out items; employees hearing a new item and requesting that the supervisor obtain a copy for review; employees coming across an item and passing it on to 
the supervisor to send it through the process; external people sending a copy of a document/ material to be considered as a new product.

This material ranges from design guidelines to state regulations to the most current field research to educational products for the general public.

When an item is obtained it goes through an initial review, which includes the following: the supervisor reviews the item for relevance to the appropriate NESC program's mission; the supervisor checks whether the item is already present in the database; the supervisor contacts the owner of the item for permission to use the item as a "Product" if it successfully goes through the review process, . an item is verified with a set of guidelines. For example, send an item to the further stages if the cost of the item to NESC is as small as negotiable, but no more than $\$ 300$, else toss the item. The item is discarded if it does not pass the initial review.

\subsubsection{TECHNICAL / PROMOTIONAL REVIEW OF ITEMS}

The basic information of items that are accepted from the initial review are entered into the database. This will include assigning a "Preliminary Accession Number", known within NESC as a product number (P\#), Source document number (EPA Doc \#) - if the item is a document, Publication Date- date an item was published/printed, date the whole internal review process starts (Date Started), number of pages (Pages)- if the item is a document, title of the item (Title), author, affiliation, medium, source.

Once all the basic information is entered into the database by the supervisor, the item is sent for technical review. The tracking of movement of an item in this process is electronic, replacing the physical movement of the "Movement Tracking Paper", known within NESC as a buck sheet along the different stages. This tracking is automatic, external to the database, located in the programming of the internal NESC server. Access is given to authorized individuals by user name and password.

In this stage three (3) technical assistants review the item individually with the use of some set guidelines and rate the item between a score of 1 - 10; 1 being bad and 10 being good. Using the above scores, and with the help of some acceptance criteria, the item is either accepted or rejected. An item which passes the acceptance criteria is given an item number determined by which NESC program reviewed it. All the necessary information is 
entered into the database, which include writing an abstract, keywords, taxonomy codes, target audience and U.S states and Countries relevant to the item. Once all the information is entered, the accepted item is sent to the promotional specialist for a promotional abstract to be written. An item which do not pass the acceptance criteria is not given an item number, but is indicated as "REJECT" in the item number field. All the necessary information of the rejected item such as abstract or keywords is entered into the database. The item is sent back to the supervisor with a note indicating the P\#.

\subsubsection{INCLUSION / PERIODIC REVIEW OF ITEMS}

The supervisor checks the information about both accepted and rejected items entered in the database for errors and has the final authority to save the record into the database. The supervisor also indicates when the next review of the item is needed. A hard copy of a rejected item is filed in the rejected file cabinet in order by its $\mathrm{P \#}$.

Bulk copies are obtained only for items that are accepted and are placed in storage. The hard copy of the accepted item is filed in the accepted file cabinet in order by its P\#.

\subsubsection{INFORMATION RETRIEVAL}

When requests for assistance about a related topic is received, the NESC staff conducts searches of the database to match products and other materials to customer needs.

\subsection{Products Database}

Environmental Health and Safety database also known as Products Database is described in this chapter. It is developed to store environmental information related to wastewater, drinking water and solid waste, specific to small communities. The fields that are used in the database are defined in detail. This part also explains a search engine that has been developed for easy information retrieval. This database was developed using RealBasic as the front end and Valentina as the backend. Since RealBasic can run on both windows and MAC-OS, the resulting database is multi-platform compatible.

Various windows/classes constitute the building of the products database. They are: Windows: 
- PRODUCTS_DATABASE1

- Taxanomy_DATABASE

- Keywords_DATABASE

- Target_DATABASE

- States_DATABASE

- Querywindow

- Technical_DATABASE

Class:

- testapplication

- Frontenddatabase

- bofrontend

A window is known as a form in Visual Basic. PRODUCTS_DATABASE1 is the main front end screen seen by the user when the database is run. Taxanomy_DATABASE, Keywords_DATABASE, Target_DATABASE, and States_DATABASE are the windows listing the taxanomy codes, keywords, target audiences and US States \& countries, relevant to review process. Querywindow is the main query window where searches on the database can be done. Technical_DATABASE is a window used by the technical reviewers to review any item that enters the technical review stage.

Frontenddatabase is a class which will manage our database. bofrontend is a class which is the child of Frontenddatase. (prefix "bo" just means base object) ( a base object means a Table in the terminology of Valentina).

\subsubsection{Definition of Terms}

The main front end screen of the database is shown below. Note that not all fields will be active for every user using the database. The Supervisor has access to all the fields. The Technical Reviewers have access only to the following fields: Abstract, Taxanomy, Keywords, Target Audience, States. The Promotional Specialist has access to Promo field only. Access is given to authorized individuals by user name and password. 


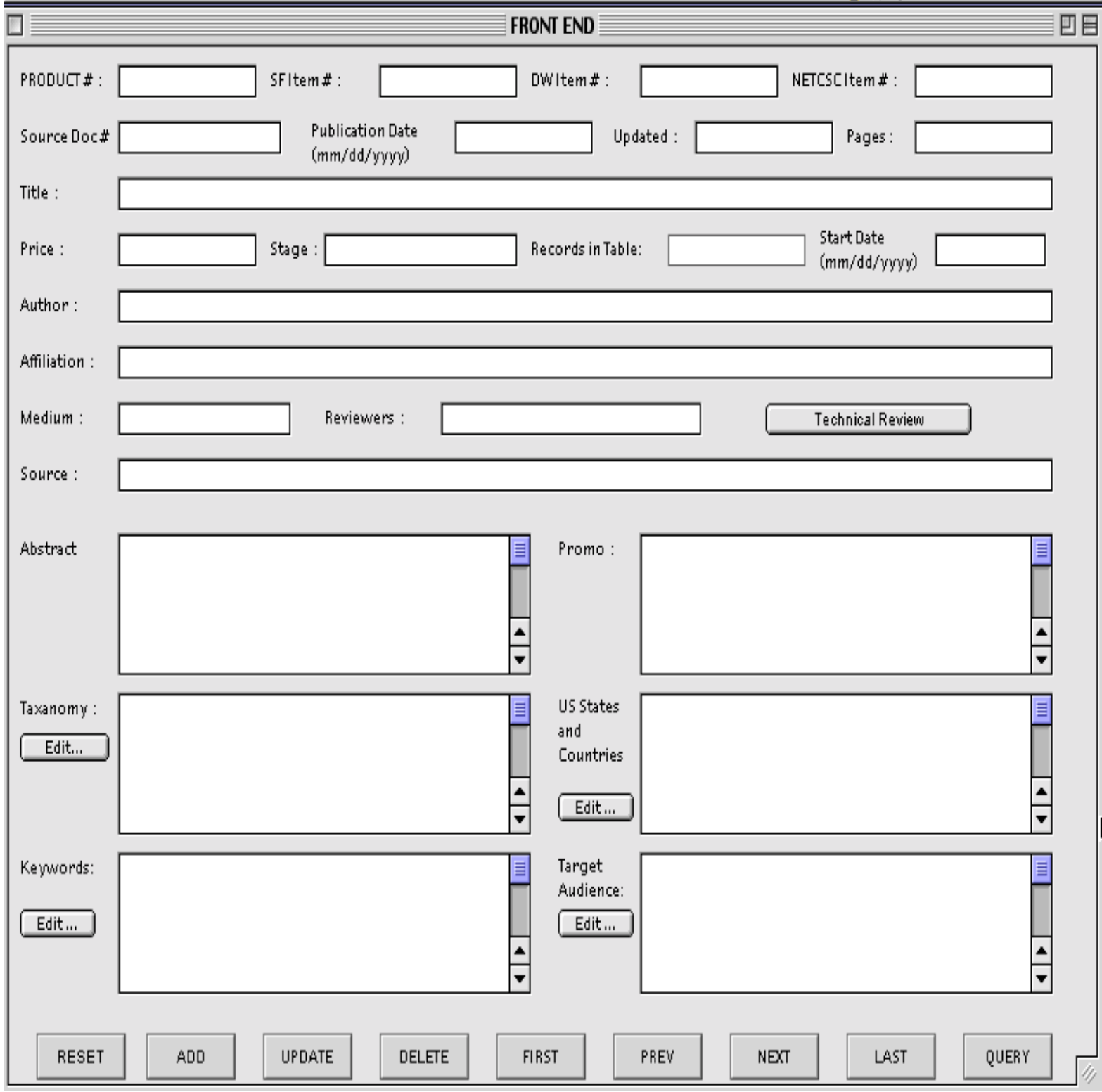

Figure 3.1: Products Database Front End Screen 
Product \#

SF Item \#

DW Item \#

NETCSC Item \#

Source DOC \#

Publication Date
This is the product number. Anything obtained to be considered for distribution as a product is given a $\mathrm{P}$ \#. This number is automatically assigned by the database.

This field represents Products offered by NSFC. This field is completed once an item has been through the review process. It contains the item number of the product once it has been accepted. If the product has been rejected in the review process, the item number will be REJECTED. If the product is no longer offered, NLA will appear in the item number. ( NLA stands for No Longer Available ).

This field represents products offered by NDWC. This field is completed once an item has been through the review process. It contains the item number of the product once it has been accepted. If the product has been rejected in the review process, the item number will be REJECTED. If the product is no longer offered, NLA will appear in the item number. ( NLA stands for No Longer Available ).

This field represents products offered by NETCSC. This field is completed once an item has been through the review process. It contains the item number of the product once it has been accepted. If the product has been rejected in the review process, the item number will be REJECTED. If the product is no longer offered, NLA will appear in the item number. ( NLA stands for No Longer Available ).

Indicates the Source Document number. For example, if the material is an EPA document, this is where you record the EPA number.

This is the date of the publication. It is what is considered as the copyright date. Entry format is $\mathrm{mm} / \mathrm{dd} / \mathrm{yyyy}$ 
Updated

Pages

Title

Price

Stage

Records in Table

Start Date

Author

Affiliation
This is the last date that any changes were made to the product information in the products database as approved by the supervisor.

This is the number of pages within the document. The product's price may be based on this page count.

This is the title of the document. This should be exactly as the document reads. This is the title that will be put in the inventory and in any publication listings.

This is the price of the document. It is calculated based on the number of pages, length of the video, amount paid to obtain the product, etc..

This field indicates the current stage of the process. They are: Initial review, Technical review, Promotional review, Information retrieval.

This field indicates the number of records in the database. This is assigned by the database.

Also known as Started. This field indicates the date the item was identified by the organization. The Entry format is $\mathrm{mm} / \mathrm{dd} / \mathrm{yyyy}$.

Up to the first 5 authors of the publication in order can be entered. Because several people may have assisted with the creation of a federal document ( USEPA, USDA, etc.. ), the federal agency \& division / department is listed as the author. For products generated within the organization, the division / unit is recorded as the author.

These are the affiliation of the author(s) and the items. For federal documents and NESC generated products, the field will be the same as that of the Author. Address would be included in this field. 
Medium

Reviewers

Source

Abstract

Promo

Keywords

Taxonomies

Target Audience
This field indicates the type of the material. ( Book, Journal, Video, etc..)

This field indicates the name of the person who initially reviewed the item or who updated it in periodic review.

This field is used to provide complete information on where to obtain additional copies for distribution if an item is offered as a product, or as a direct reference to customers if the item is not offered as a product.

This field indicates an abstract of the purpose and contents of an item. This field is filled after an item has been reviewed regardless of whether or not accepted.

This field indicates the promotional abstract about an item which the NESC uses for promotional activities. This field is filled for items which are accepted after the technical review.

This field lists the keywords related to the product. It is a pull down list. Only words listed on the list are available for use as keywords.

The taxonomies code field is also a pre-selected pull down list that can be used to perform searches. This field is used only by NSFC .

This field lists which audience bases are expected to find the product most useful. This is also a Pull down list. 
US States and and Countries

Technical Review

ADD

UPDATE

DELETE

FIRST

PREV

NEXT

LAST

QUERY
This field is used to record the US State(s) and Foreign Countries that are referenced within the product. This would include any and all cases studies mentioned, regulations / policies / procedures specific to a state or states.

This button takes us to a different window which is used for technical review

This adds a new record to the database

This updates or edits an existing record

By pressing this button, the current record can be deleted.

The first record is pulled up when this button is pushed

The record, previous to the current one is pulled up when this button is pressed.

The next record is pulled up when this button is pressed.

The last record in the database is displayed when this button is pushed.

A different search window is displayed. 


\subsubsection{Search Engine}

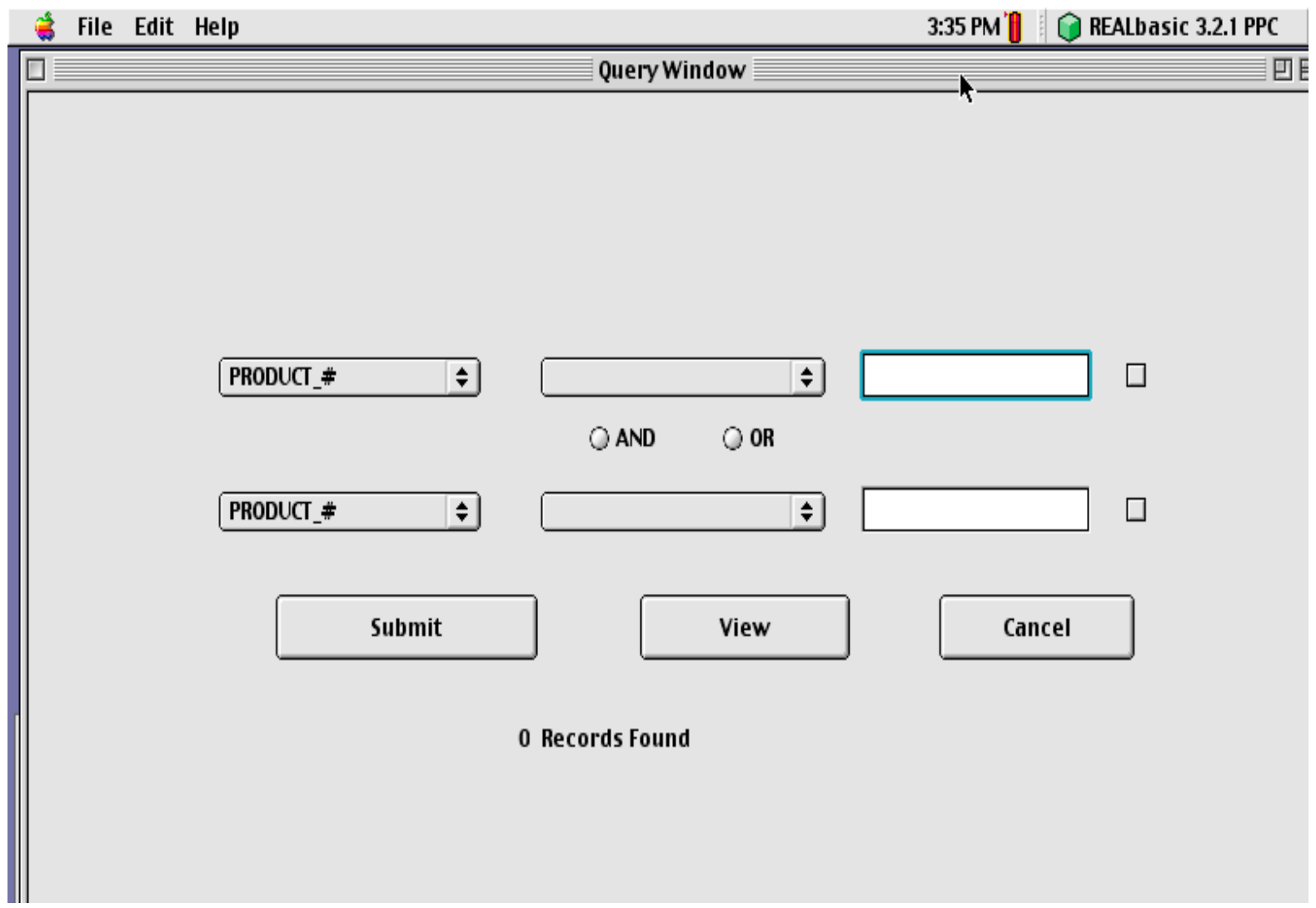

Figure 3.2 Search Engine Screen

PopUp Menu : $\quad$ The "Product \#" menu gives a selection of the fields on which we can perform a search, such as Product \#, SF Item \#, DW item \#, NETCSC item\#, etc..

PopUp Menu: $\quad$ The second pop up menu which is to the right of PopUp menu (Product_\#) gives a selection of the criteria on which the search can be based, such as Equal to, Greater than, Less than.

Radio Button: $\quad$ Selection of boolean operators to combine searches logically. 
Submit:

Once the search criteria are entered, upon pressing this button, the number of records the search engine pulls up is displayed below.

View: $\quad$ Once the search is done and the record(s) is/are found, view takes us to a different screen which will display the current searched record(s).

\subsection{Implementation}

This part explains a step - by - step procedure in details: how to use the database.

When the RealBasic icon $\mathbf{D}$ is double clicked the following screen is displayed as seen in Figure 3.3.

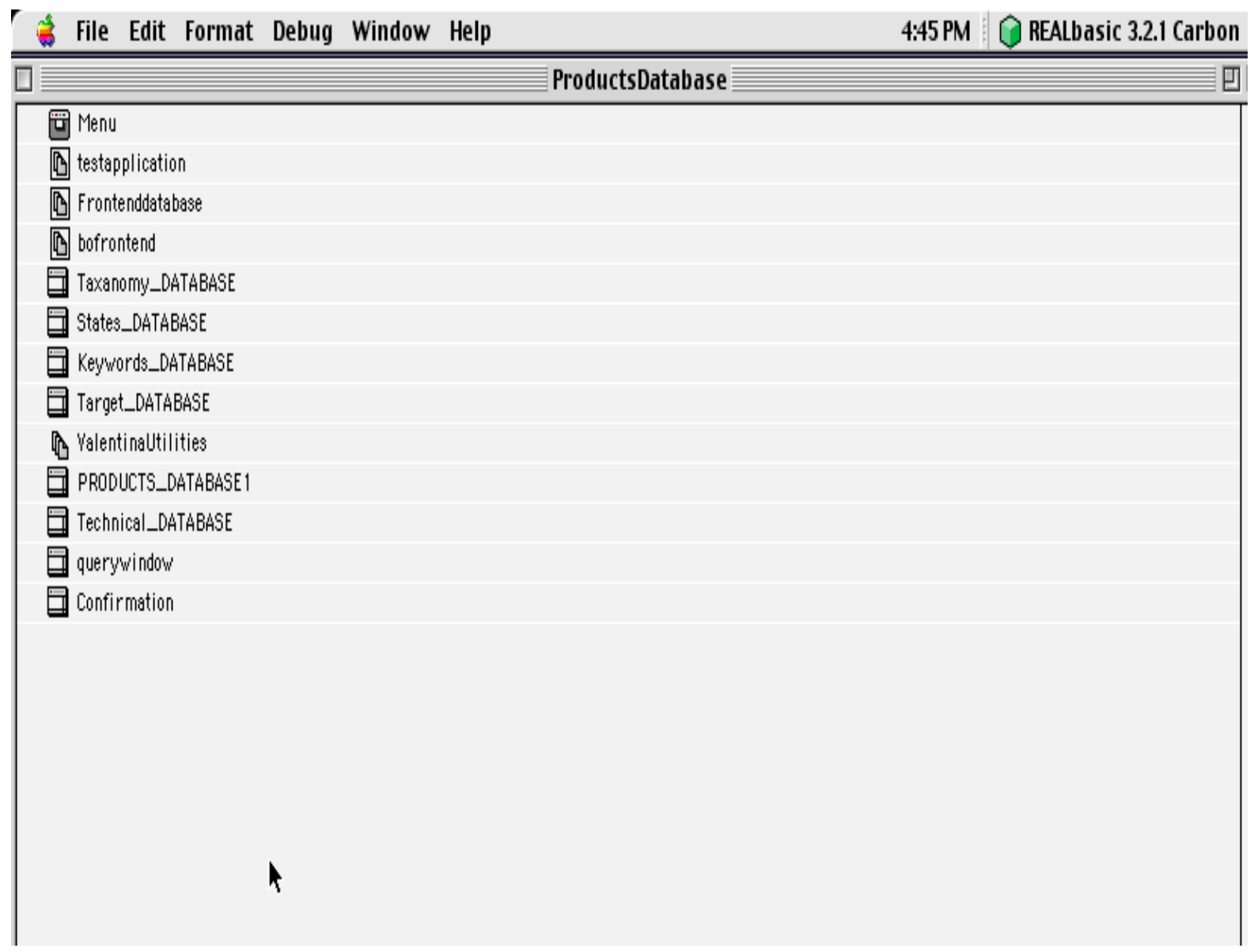

Figure 3.3: Opening Screen 
To run the application we go to Debug $\rightarrow$ Run as seen from Figure 3.4

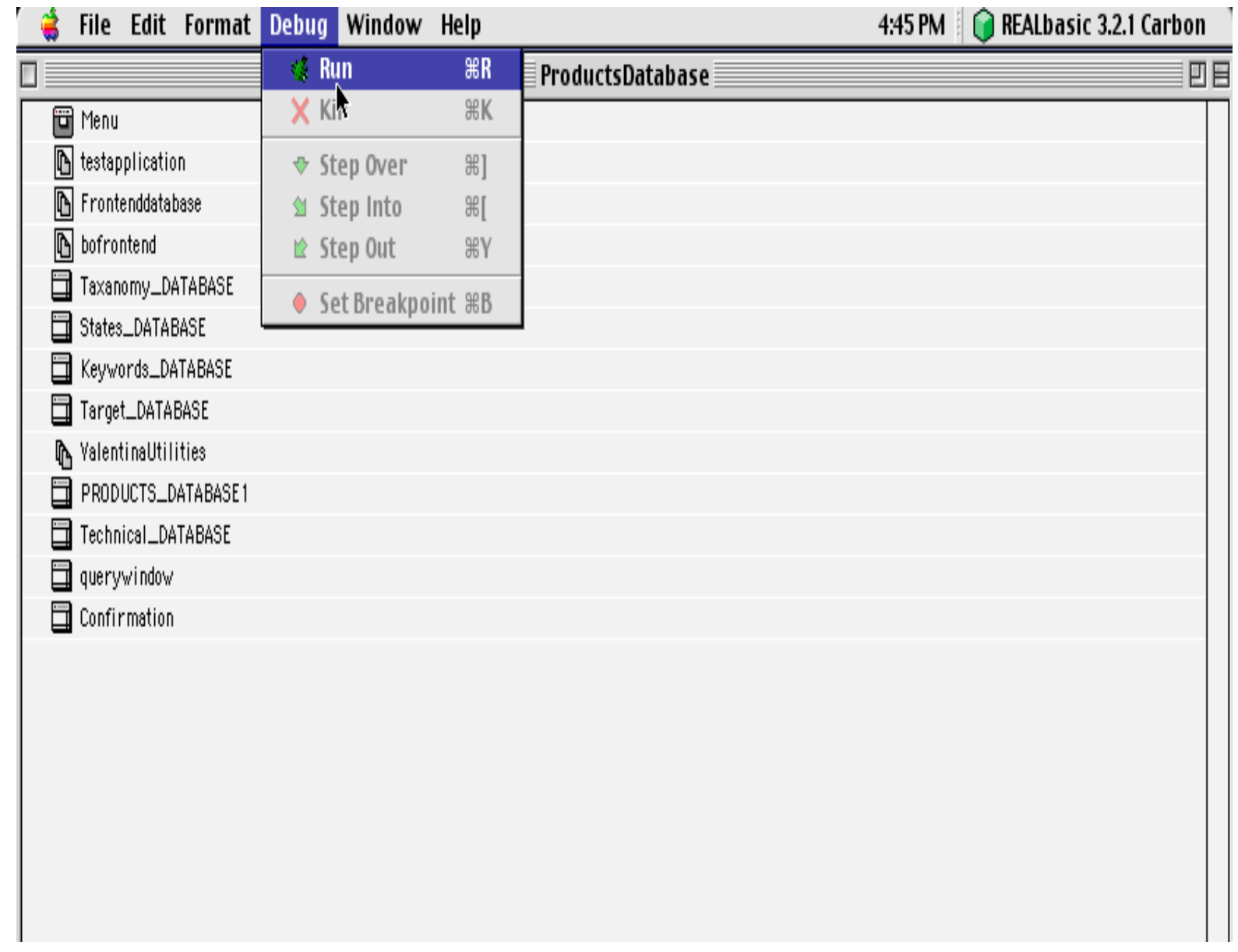

Figure 3.4: Screen showing the Run button

The drop down menus from the above figure are part of the RealBasic programming options and are not relevant to our database except for the Debug $\rightarrow$ Run menu as seen from above. Clicking on Run opens the window shown below as seen in Figure 3.5. The screen has the following Menus

File $\rightarrow$ New, Open, Quit

Edit $\rightarrow$ Undo, Cut, Copy, Paste, Clear

Help 


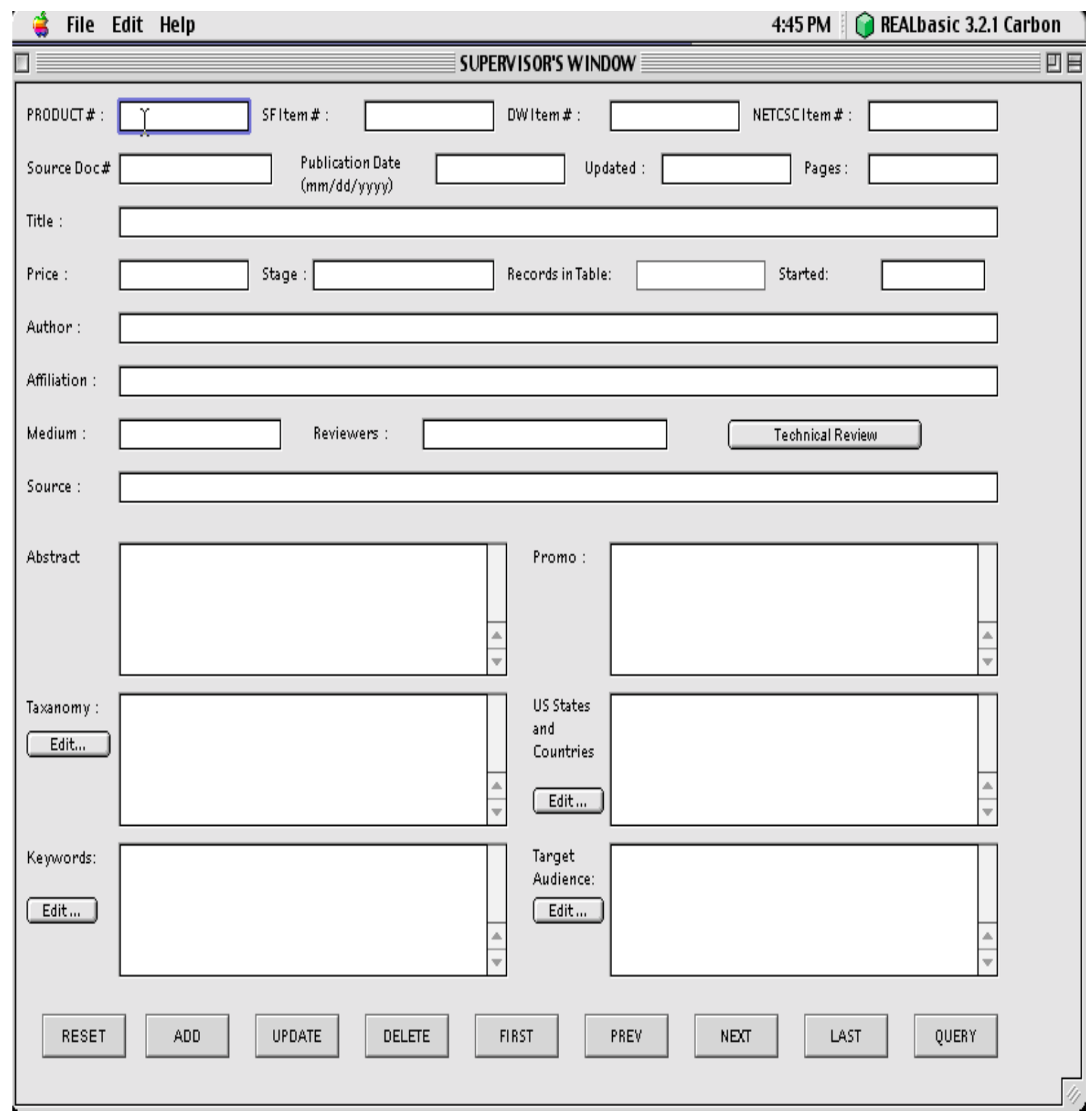

Figure 3.5: Main Front End Screen

To create a new database click on File $\rightarrow$ New as seen in Figure 3.6. To open an existing database click on File $\rightarrow$ Open 


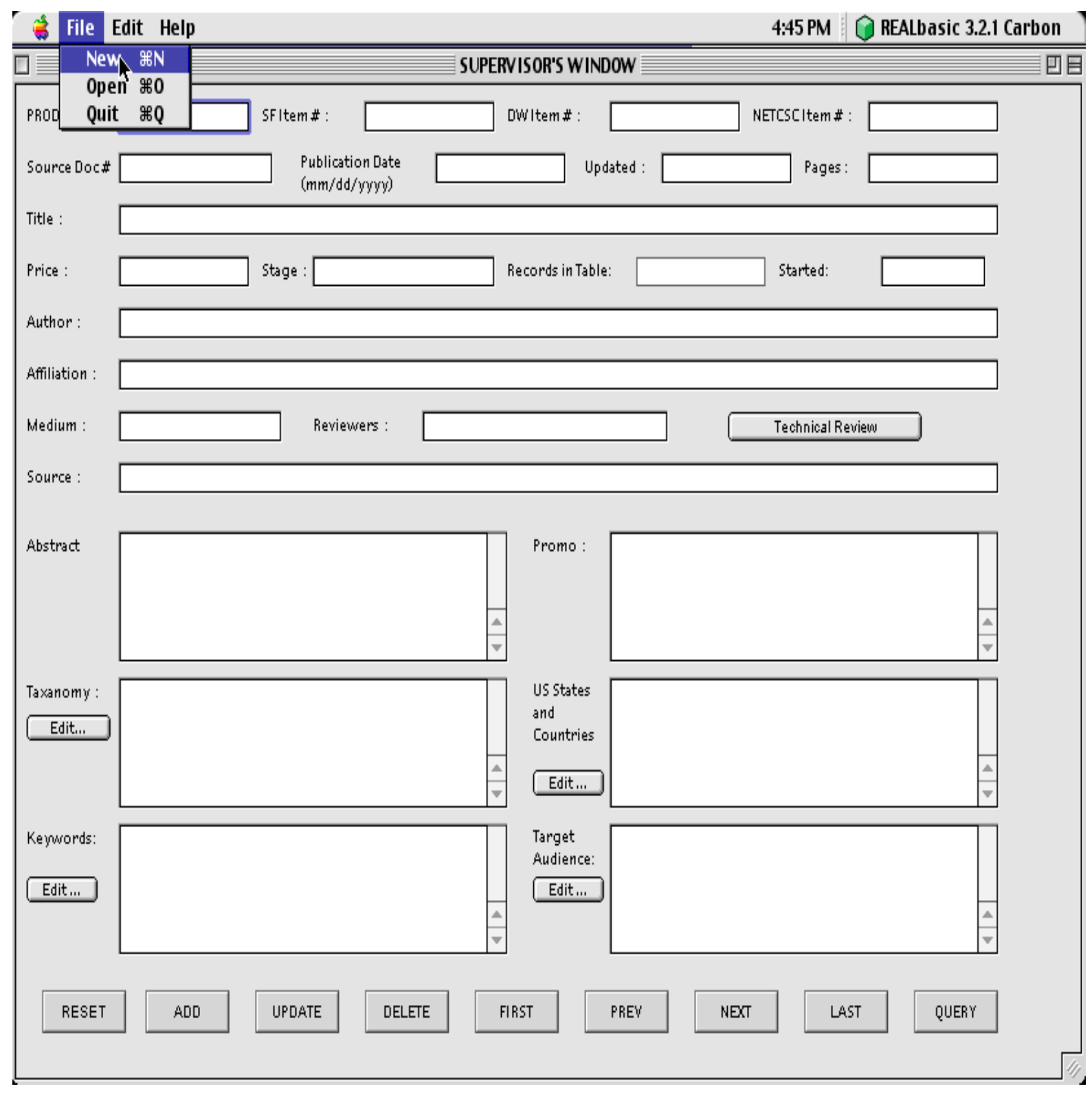

Figure 3.6: Screen showing the File Menu

When File $\rightarrow$ New is clicked, a new database is created and a screen is displayed as seen in Figure 3.7, in which the name of the database is to be entered. 


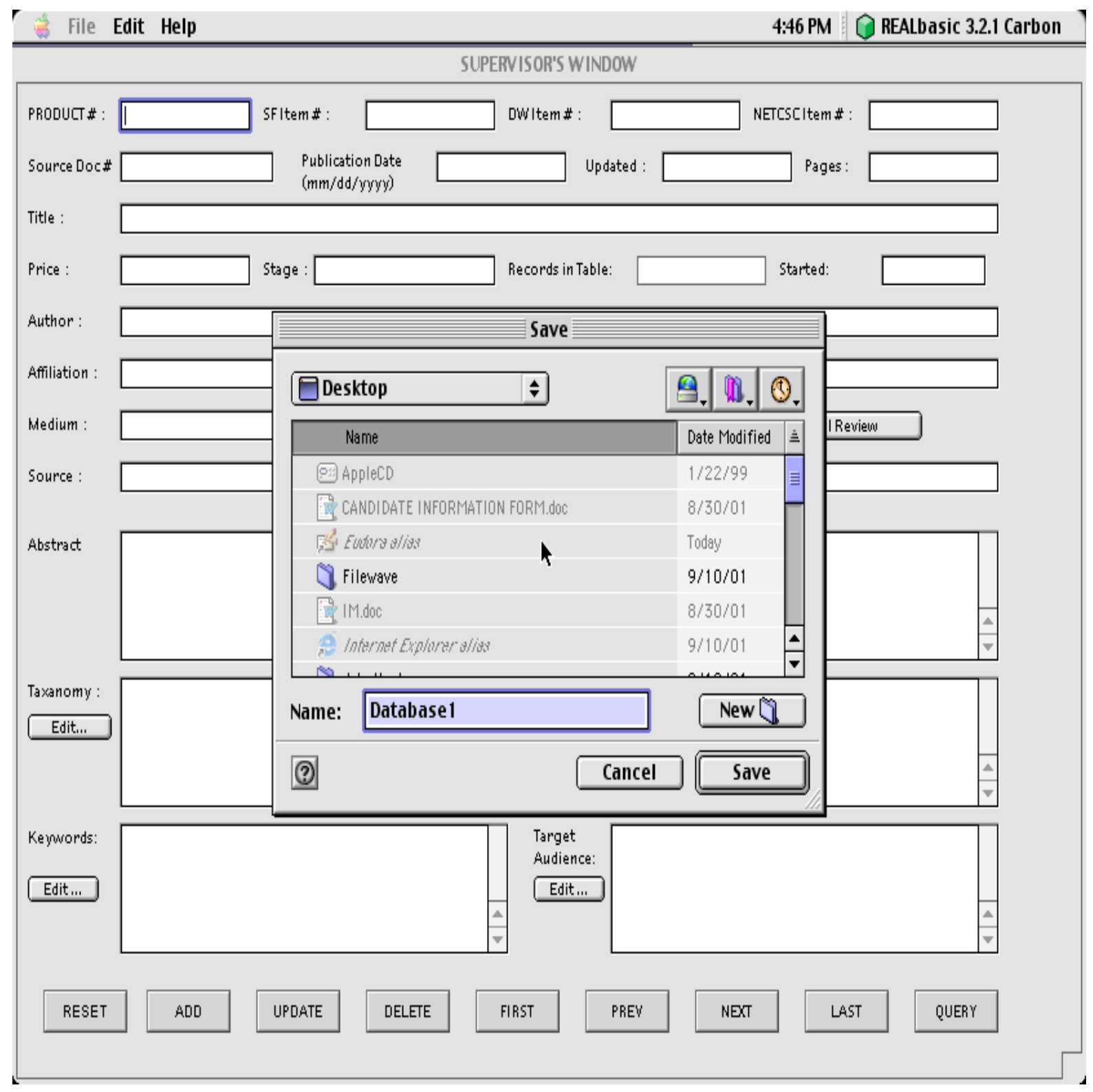

Figure 3.7: Create New Database Window

Once the name of the database and the path to the location at which it needs to be saved, is decided, and the Save button is clicked, a new window is displayed as seen in Figure 3.8. 


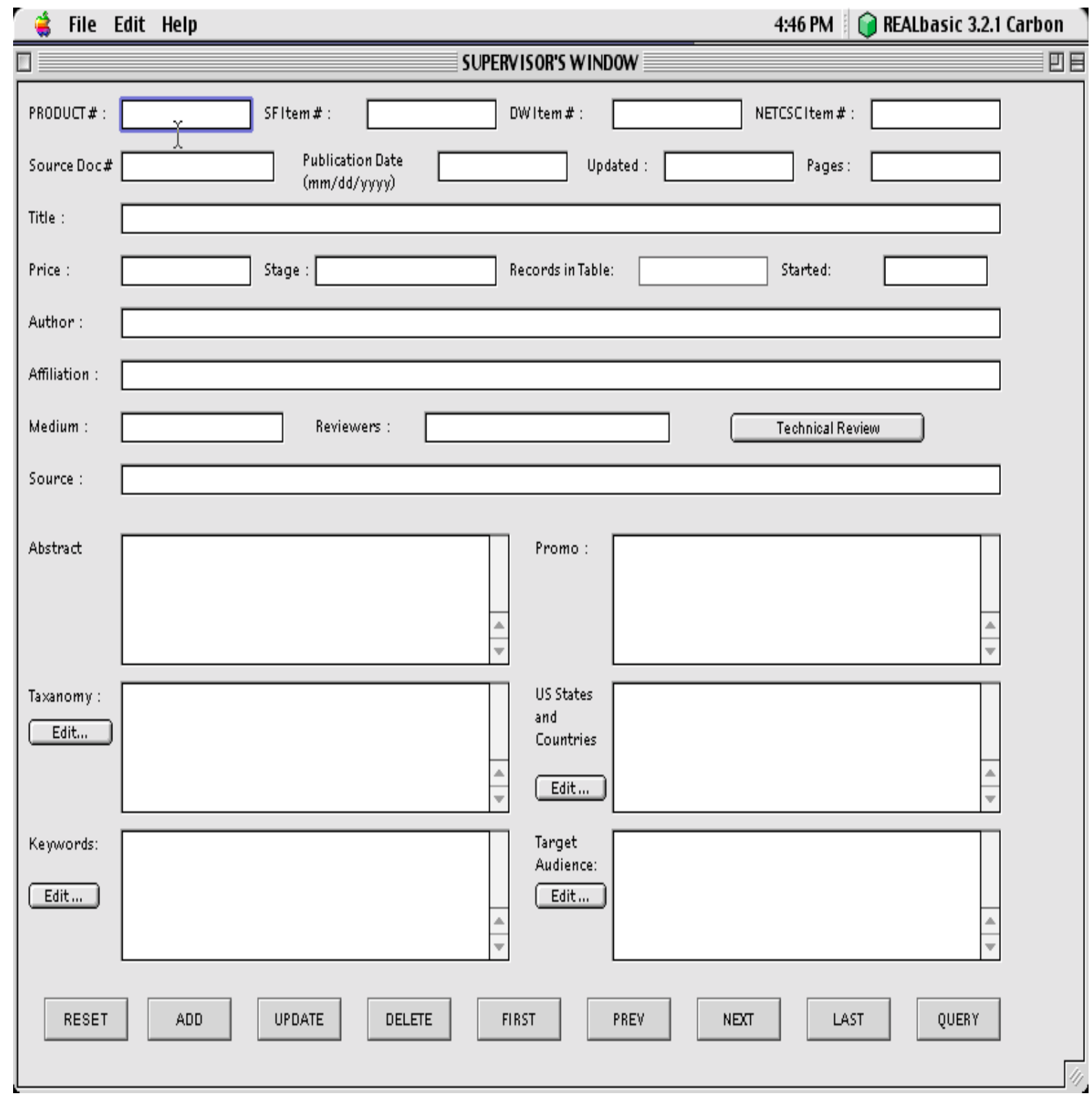

Figure 3.8: Data Entry Form

The supervisor starts the product review process. Once the initial review of an item is completed, and the item passes the initial review, a product number is assigned for the item and all the basic information is entered into the database as shown in Figure 3.9 below. The screen below is the screen seen by the Supervisor who has access to all the fields. 


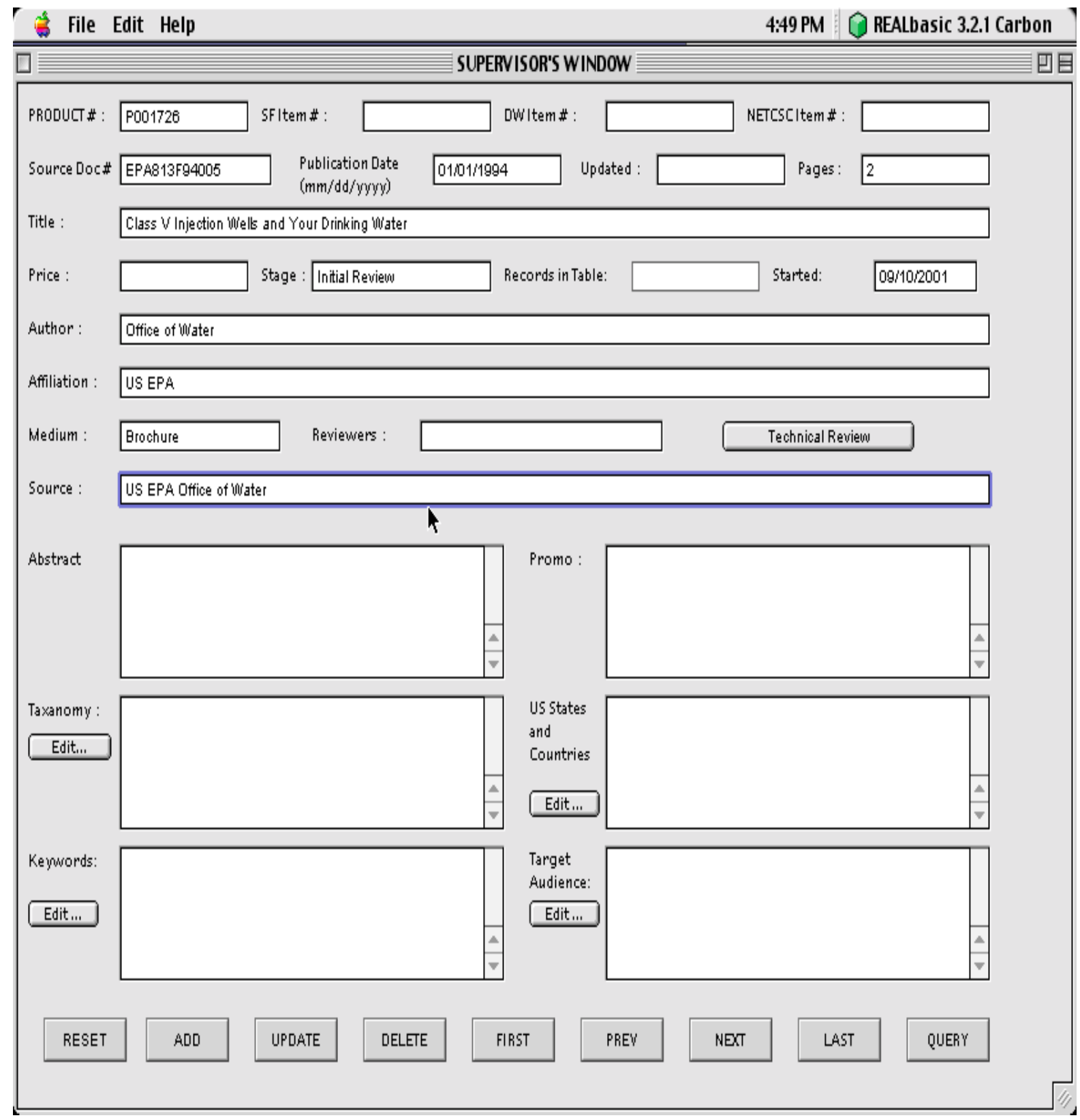

Figure 3.9: Screen showing Basic Information of an item

Once all the basic information of the item is entered, the item is sent for technical review. This marks the beginning of the Technical Review Stage. At this stage the Technical Reviewer has access only to click on the Technical Review Button . In the technical review, one or more technical reviewers review the item and score the item 
according to 5 particular criteria. Clicking on the Technical Review button opens a new window as shown below.

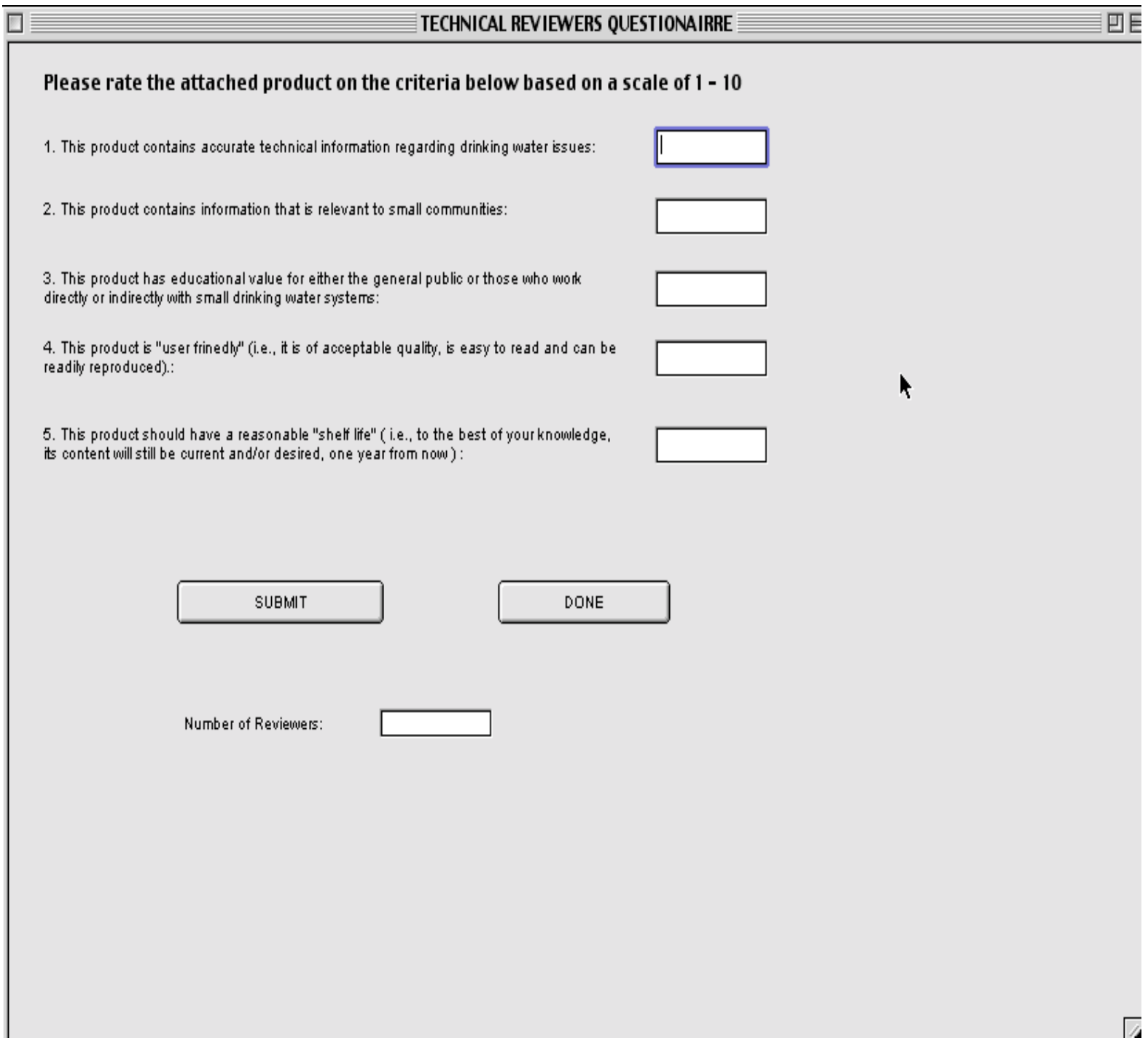

Figure 3.10: Technical Review Questionnaire Window

Technical Reviewer scores the item on each of the 5 criteria, on a scale of $1-10,1$ being worst and 10 being best, as seen in Figure 3.11. 


\section{TECHNICAL REVIEWERS QUESTIONAIRRE}

\section{Please rate the attached product on the criteria below based on a scale of $1-10$}

1. This product contains accurate technical information regarding drinking water issues:

$$
10
$$

2. This product contains information that $i$ relevant to small communities:

\section{9}

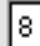

3. This product has educational value for either the general public or those who work directly or indirecthy with small drinking water systems:

4. This product is "user frinedb" (i.e., it is of acceptable quality, is easy to read and can be readity reproduced):

5. This product should have a reasonable "shelf life" ( i.e., to the best of your knowledge, its content will still be current andior desired, one year from now):

8
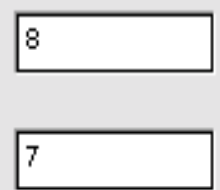

7

10

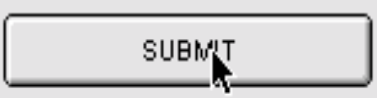

DONE

Number of Reviewers:

Figure 3.11: Technical Reviewer Score

Once the first reviewer gives a score to all the 5 criteria, he clicks on the submit button which marks the end of technical review 1 and all the fields are reset as blank for the second reviewer to review the item as seen in Figure 3.12, and note that the Number of Reviewers field is incremented by 1 . 


\section{Please rate the attached product on the criteria below based on a scale of 1 - 10}

1. This product contains accurate technical information regarding drinking water issues:

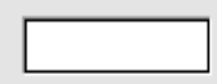

2. This product contains information that is relevant to small communities:

3. This product has educational value for either the general public or those who work directly or indirectly with small drinking water systems:
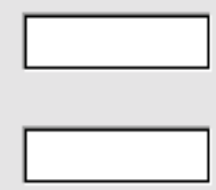

4. This product is "user frinedby" (i.e., it is of acceptable quality, is easy to read and can be readity reproduced).:

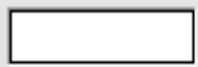

5. This product should have a reasonable "shelf life" ( i.e., to the best of your knomledge, its content will still be current andior desired, one year from now ):
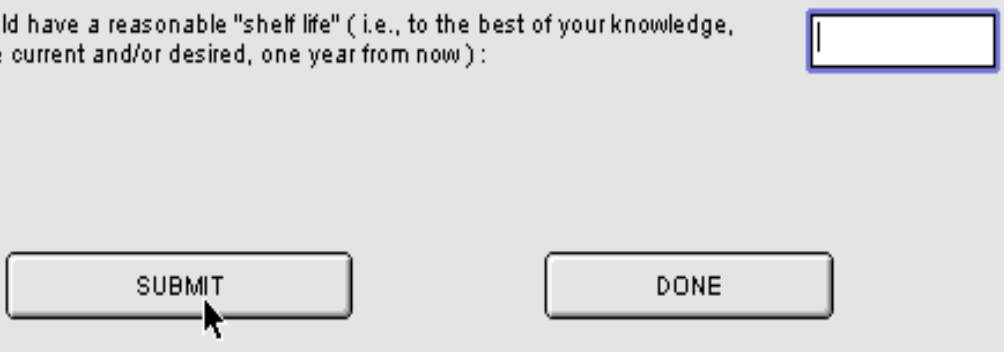

DONE

Number of Reviemers:

1

Figure 3.12: Window for Second Technical Reviewer

The second and third reviewers review the same item and each gives a score on a scale of 1 10 for each of the 5 criteria. Once all the 3 reviewers are done with reviewing, the DONE button is clicked. Upon clicking the DONE button, a message box pops up which indicates the results of the technical review based on some automated acceptance criteria. For our case, if the average of the scores given by the technical reviewers is more than or equal to 40 , the item is automatically accepted. The screen is shown in Figure 3.13. 


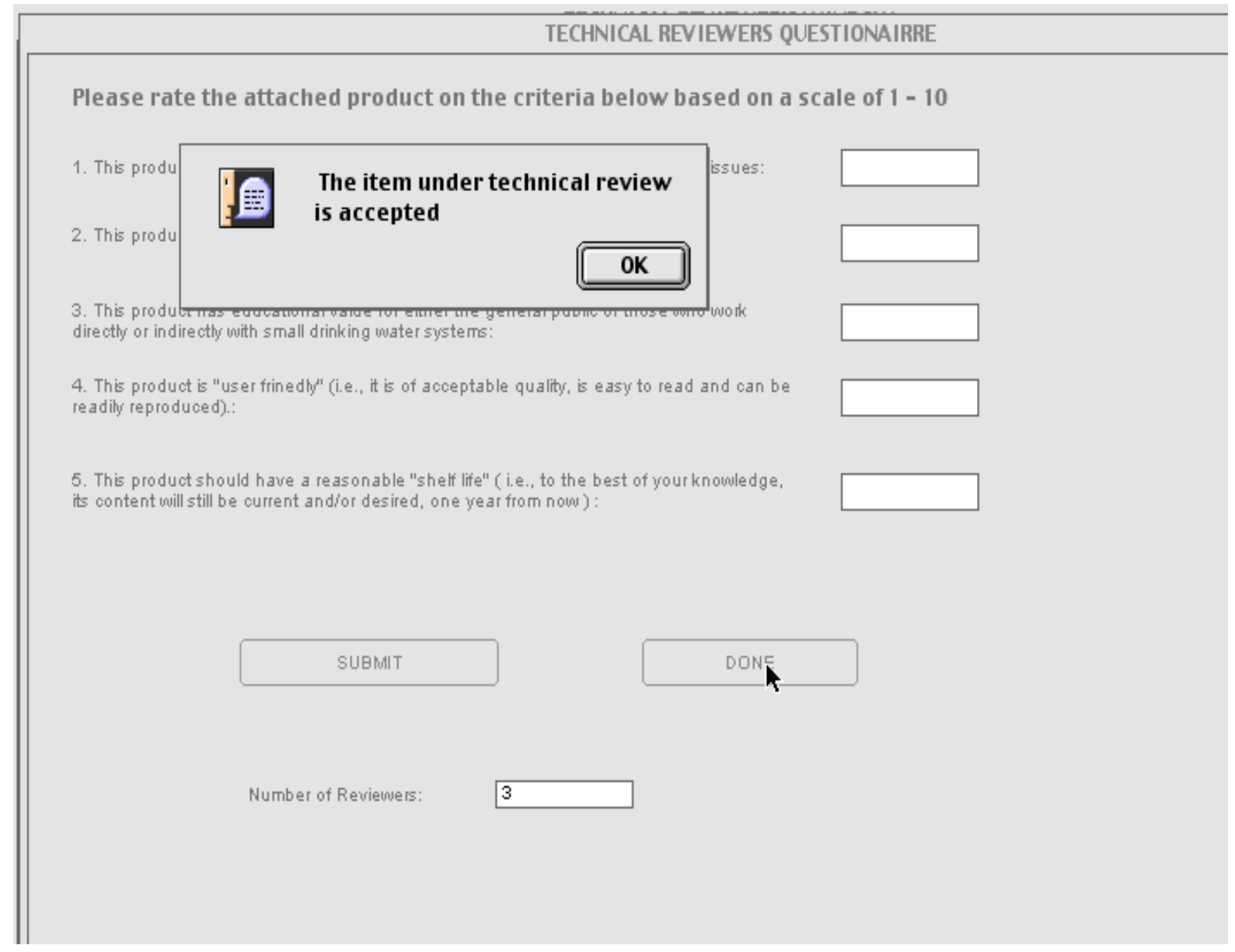

Figure 3.13: Technical Review Result Window

If an item is rejected, a message box pops up which indicates "The item under technical review is rejected because it does not pass the acceptance criteria".

Clicking the OK button on the message box takes us back to the main window. Note that at this stage the technical reviewer has access to the following fields: Abstract, Keywords, Taxanomy Codes, US States and Countries, and Target Audience. They do not have write access to any other fields. At this stage, an item number is assigned depending on the organization that reviewed the item. The screen is as shown in Figure 3.14. 


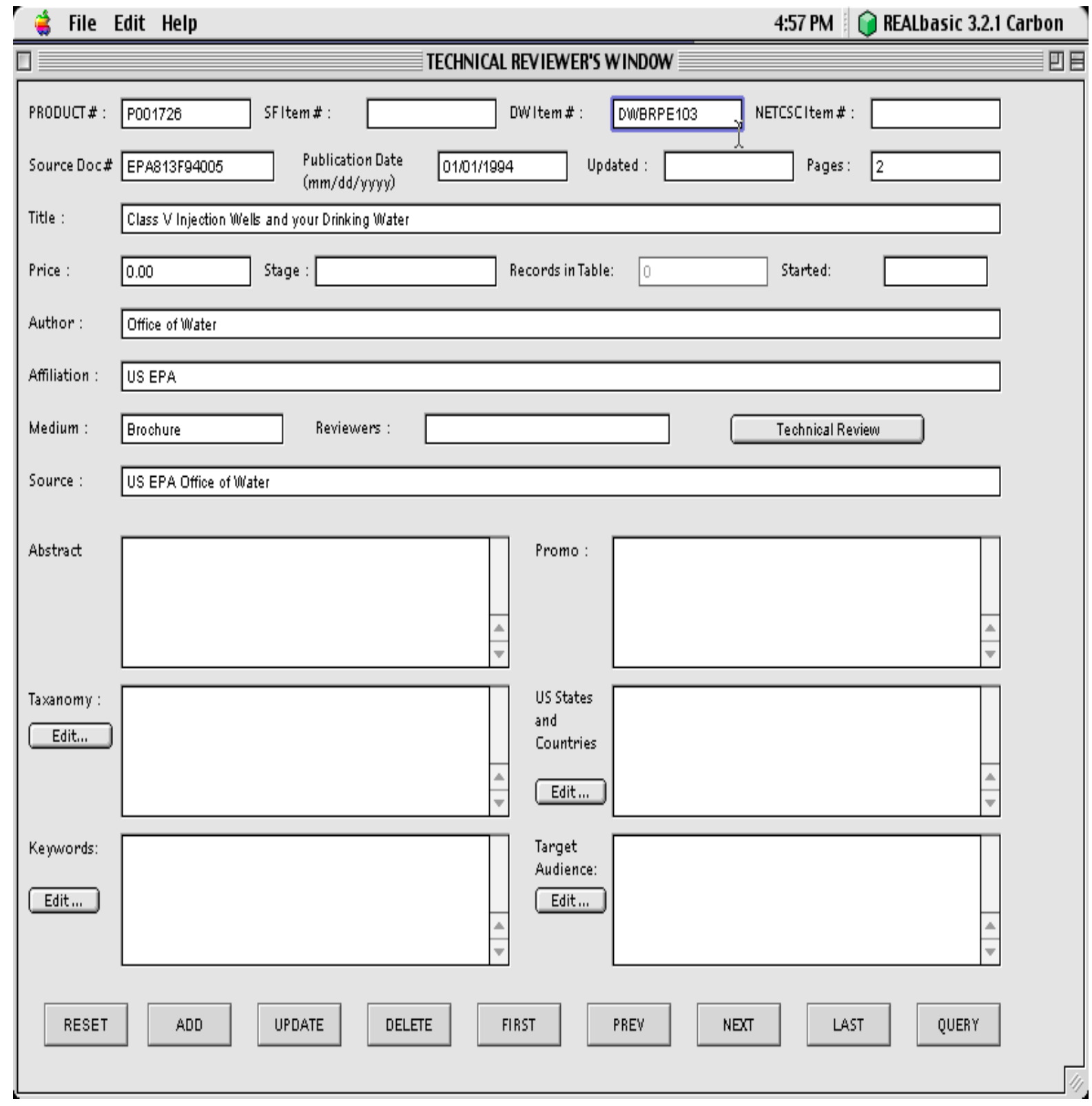

Figure 3.14: Item Number Entry Screen

Once the item number is assigned, the Abstract of the item is keyed into the Abstract field as shown in Figure 3.15 


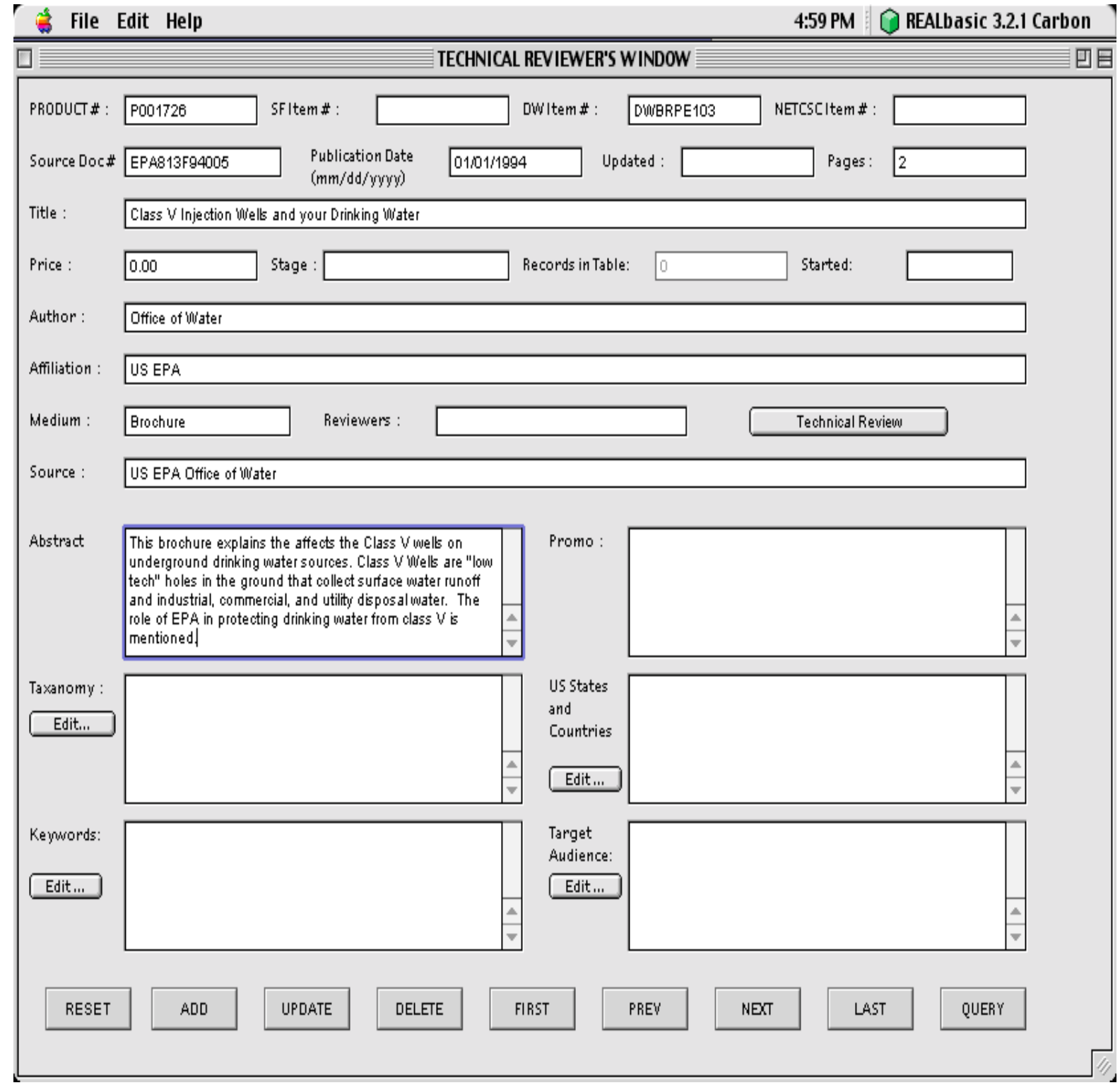

Figure 3.15: Abstract Field Entry Screen

The next step is to add the keywords relevant to the item. To do so the edit button under the keywords is clicked on as shown in Figure 3.16 


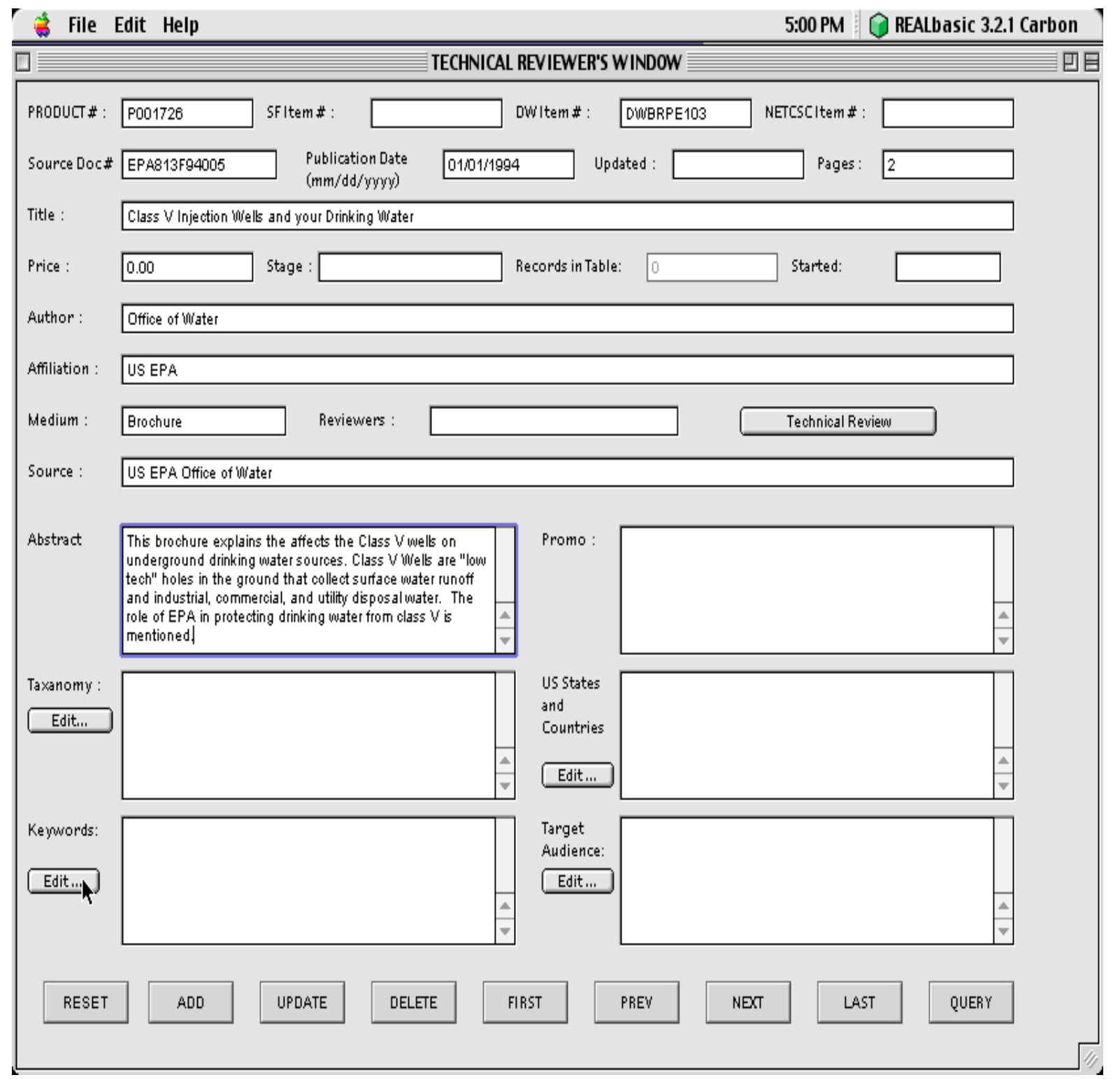

Figure 3.16: Keyword Edit Click Window

Once the edit button under the field Keywords is clicked, a new window pops up as shown in Figure 3.17 


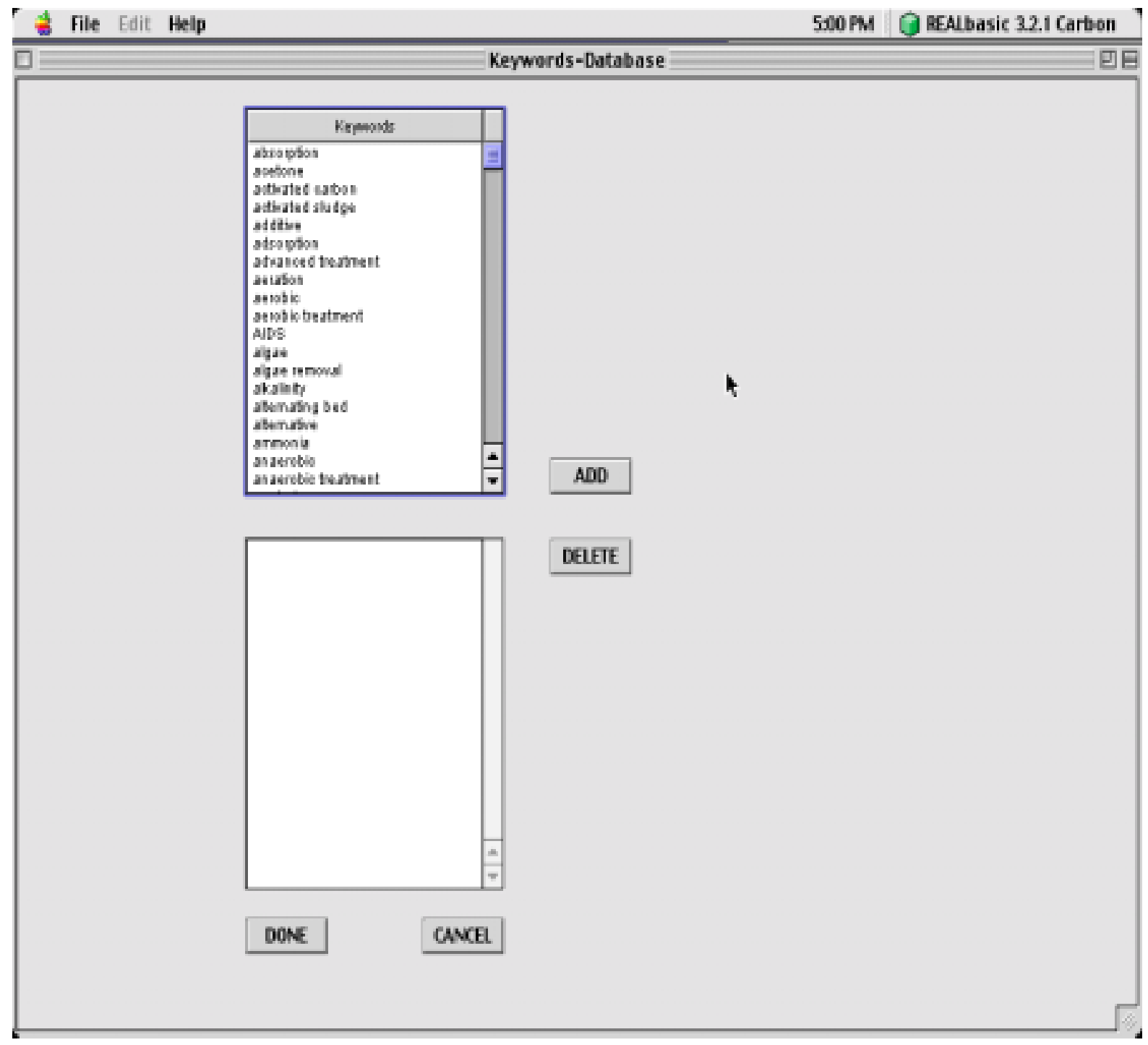

Figure 3.17: Main Keyword Window

The relevant keywords are found by scrolling and is selected. On clicking the ADD button, the keyword is selected and is displayed in the lower window as seen in Figure 3.18. Similarly all the needed keywords are added. The DELETE button deletes the keywords from the lower window. 


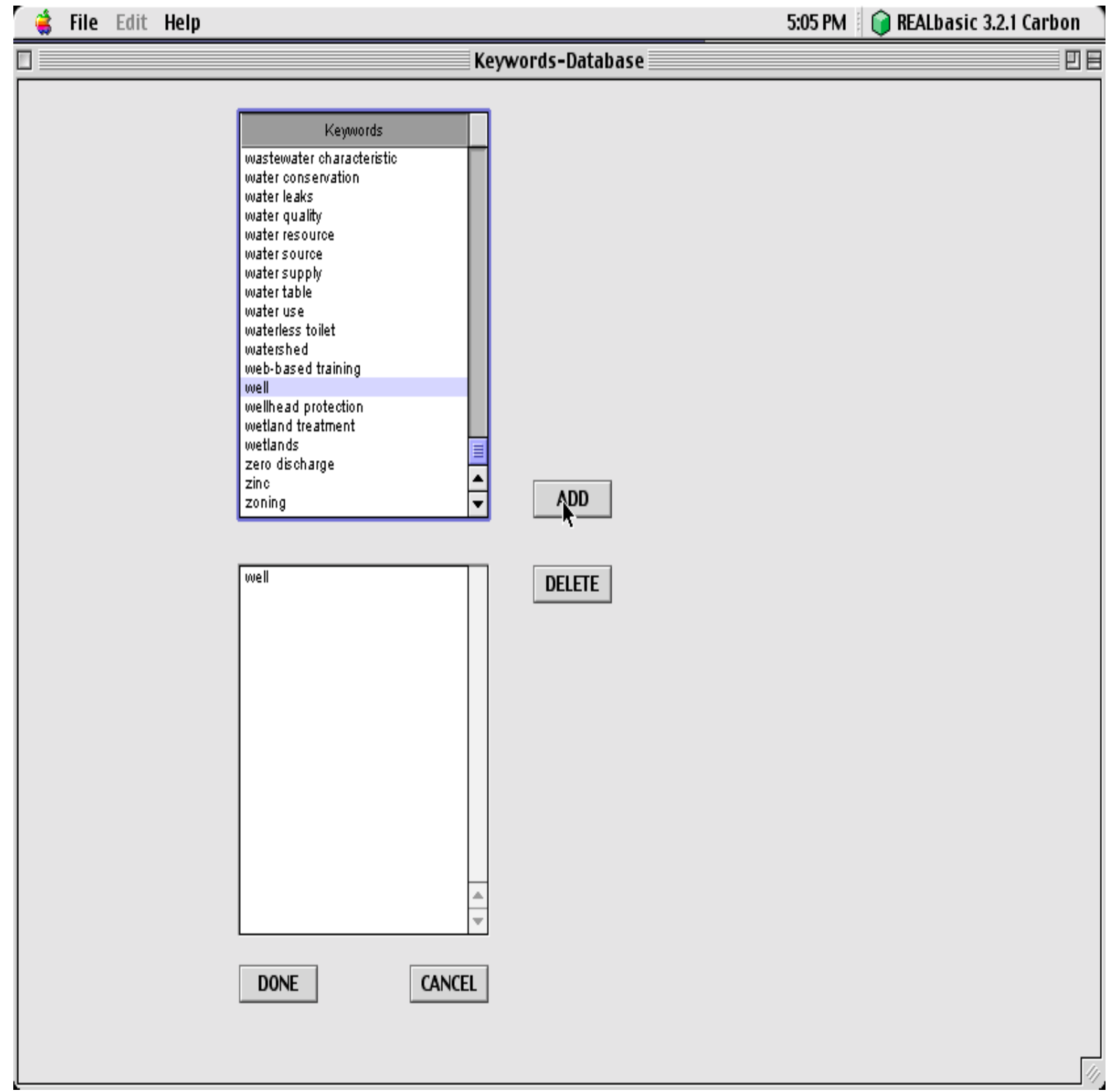

Figure 3.18: Keyword Select Window

Upon clicking the DONE button, the selected keywords from the lower window is carried over to the main window as seen from Figure 3.19 


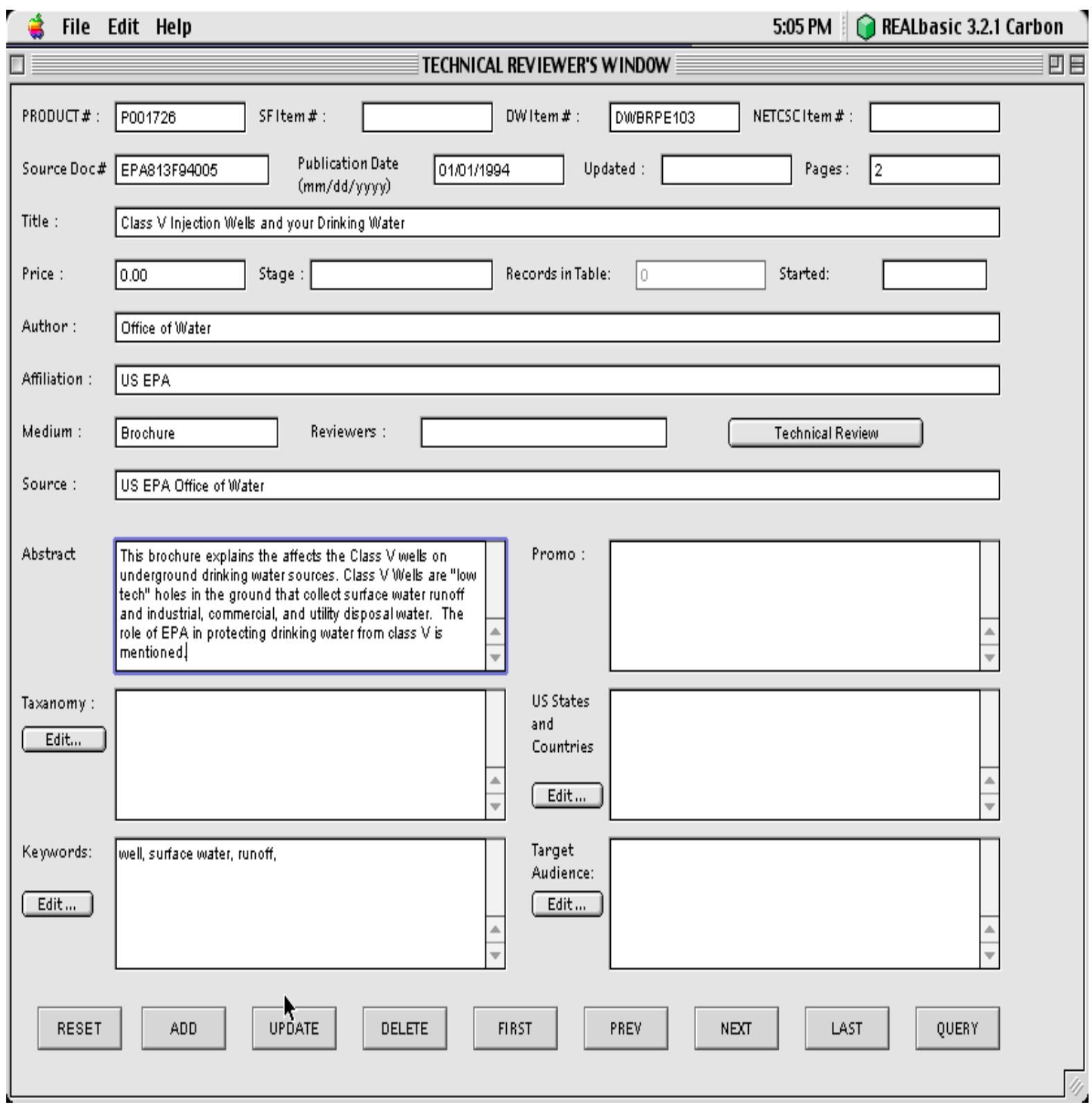

Figure 3.19: Selected Keywords Display Screen

To add Taxanomy codes, the edit button below the field Taxanomy is clicked. Upon clicking the edit button, a new window appears as shown in Figure 3.20 


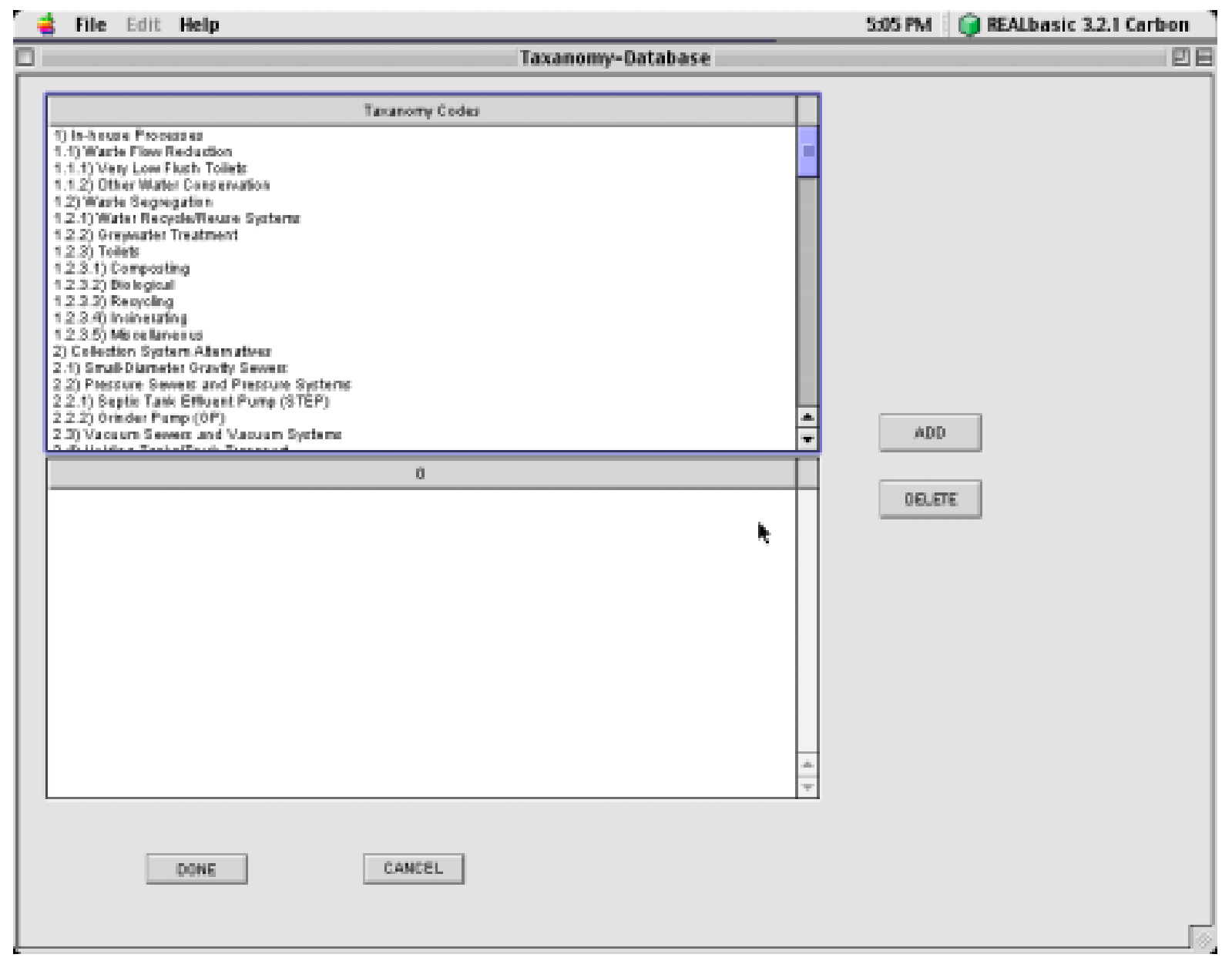

Figure 3.20: Main Taxanomy Window

The taxanomy codes are selected and the ADD button is clicked which displays the selected code in the lower window. The organization which is reviewing this item ( NDWC) does not use taxanomy codes. Pressing the DONE button returns us to the main window.

To add states relevant to the item, the edit button below states is clicked on which takes us to a screen as seen from Figure 3.21 


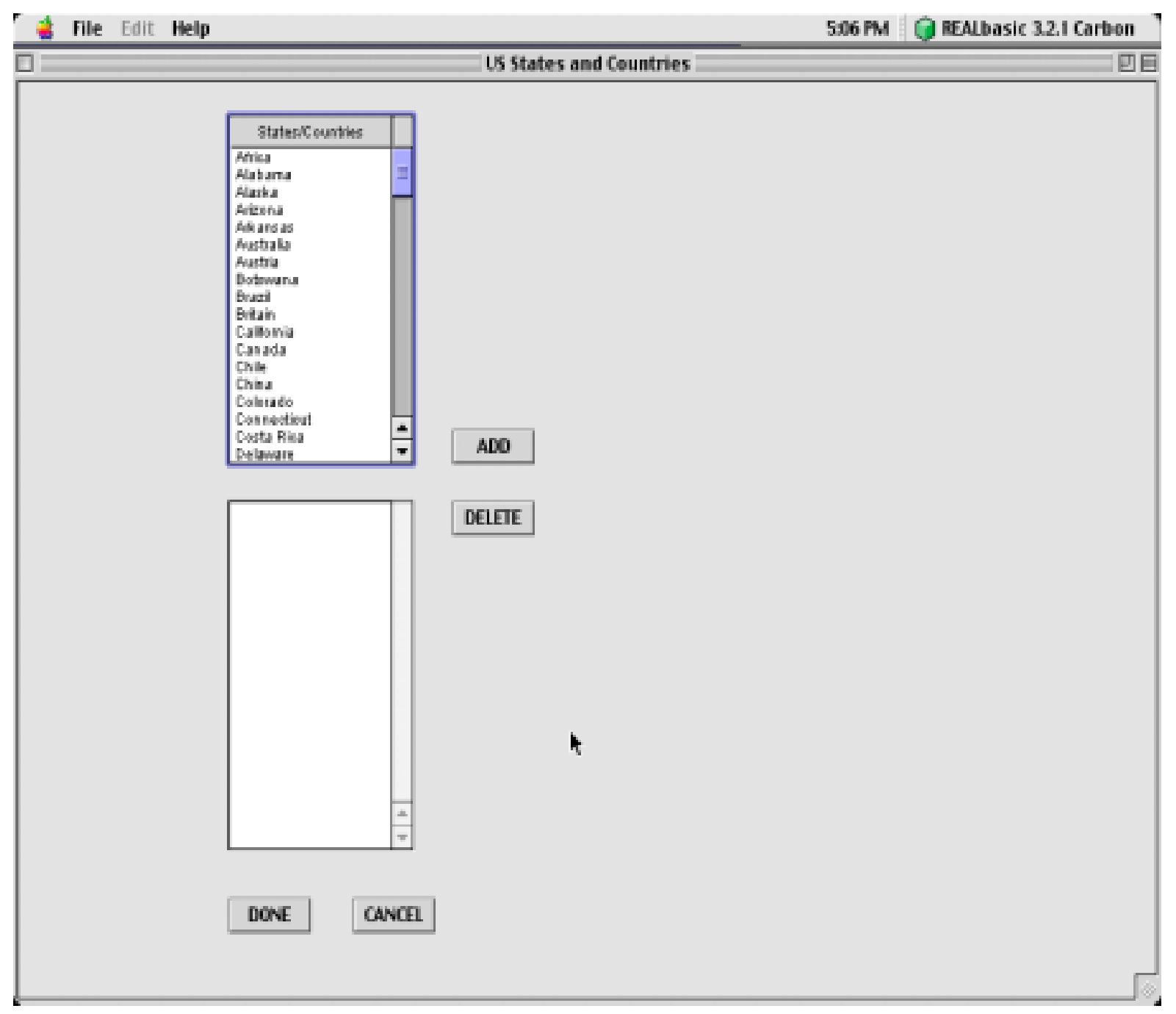

Figure 3.21: Main States Window

The item under review is not relevant to any state as such, no states are selected.

To add the target audiences relevant to the item, the edit button below the field Target Audience is clicked on which takes us to a screen as shown below in Figure 3.22. 


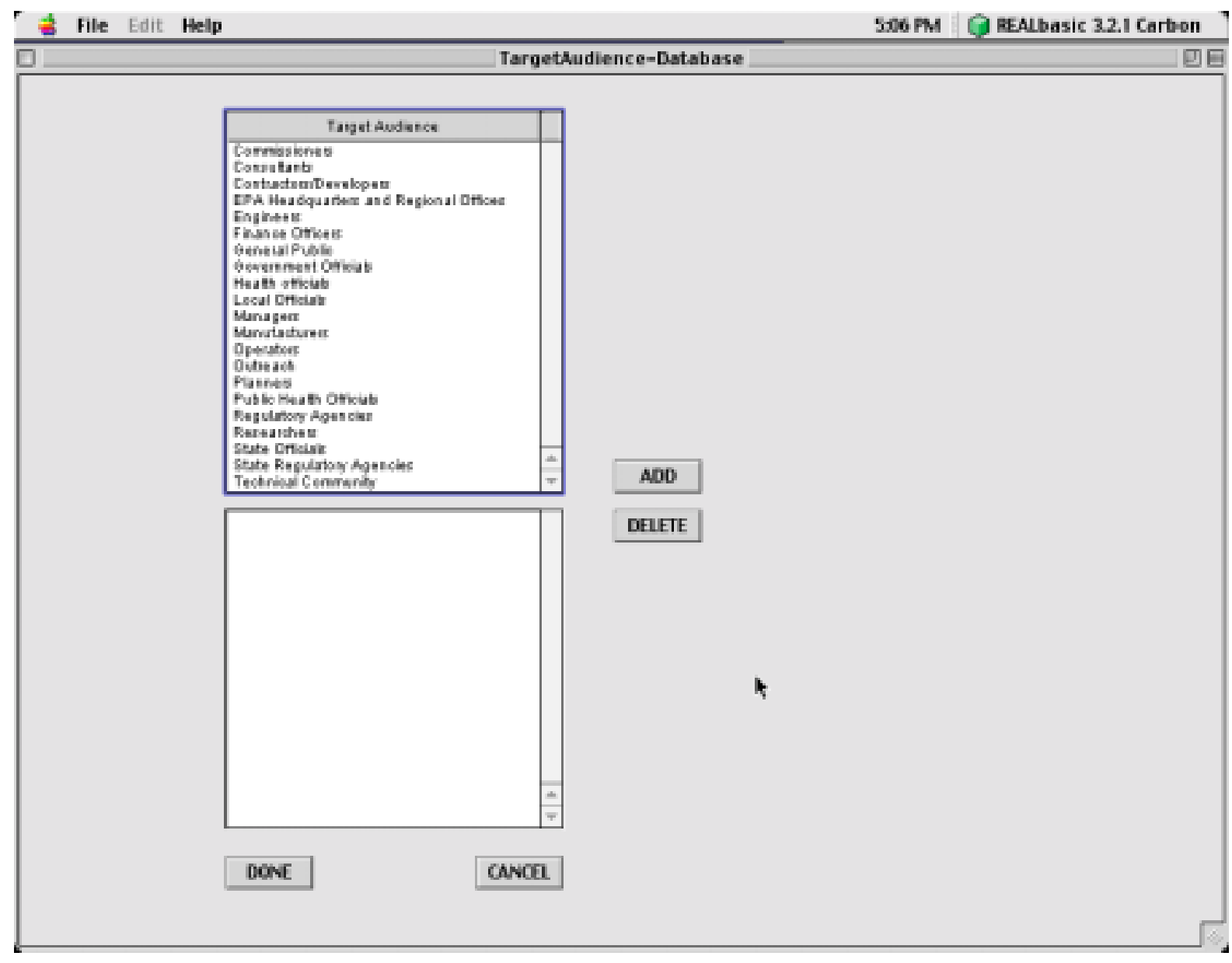

Figure 3.22: Main Target Audience Window

Once the relevant target audience is added and the DONE button is clicked, it takes us to main window.

This marks the end of technical review. The Promotions Specialist is notified that the item is ready for a promotional abstract to be written. The item is sent to the promotional specialist for a promotional abstract to be written. The promotional specialist writes the promotional abstract for the item in the Promo field as shown in Figure 3.23. 


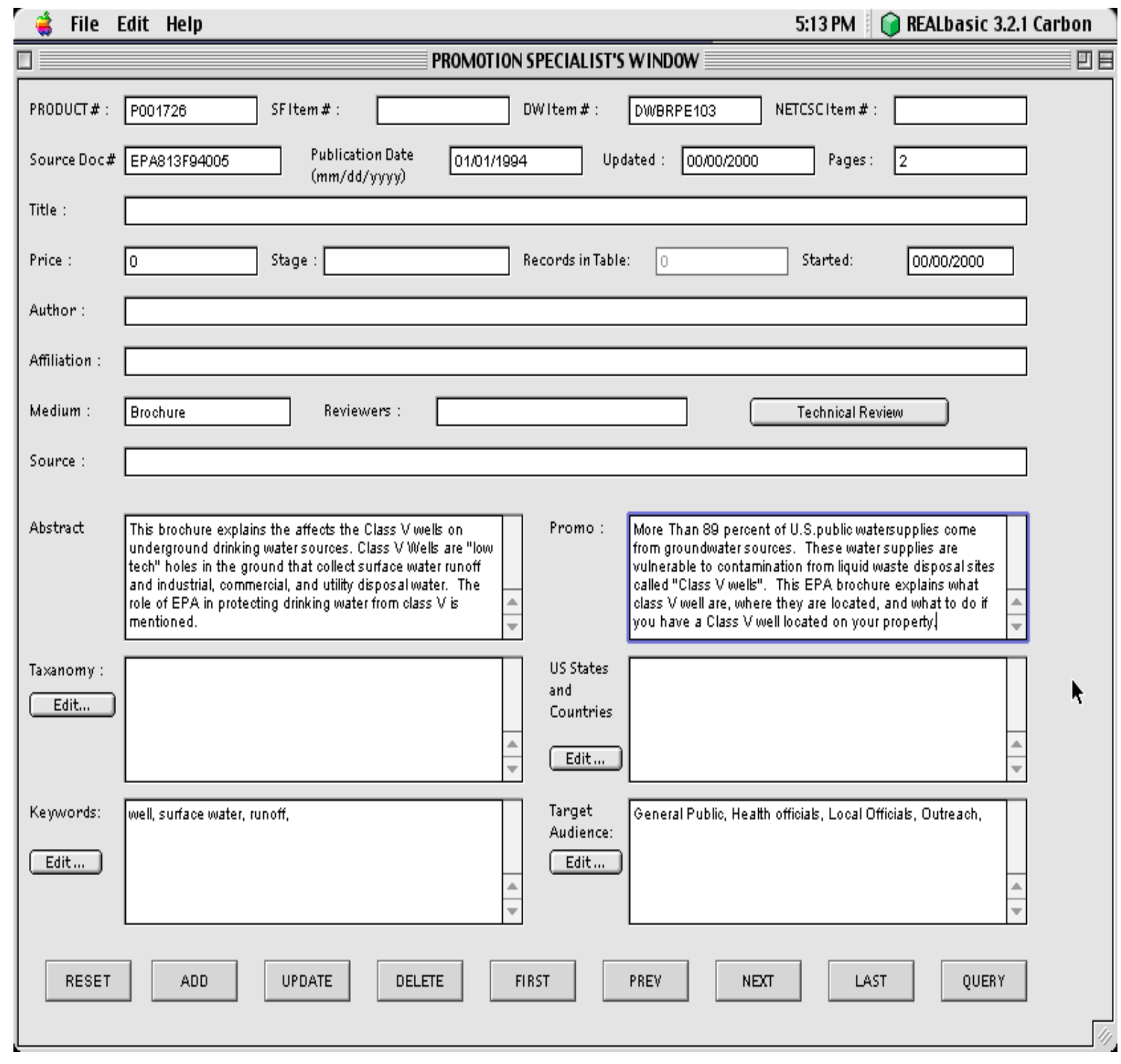

Figure 3.23: Promotions Entry Screen

Once the promotional abstract is written, the supervisor is notified. The supervisor checks the record for errors and has the final authority to save the records. 
The supervisor clicks on the ADD button to add the record into the database. Upon clicking the ADD button, the record is added into the database and all the fields in the main window are cleared as shown below.

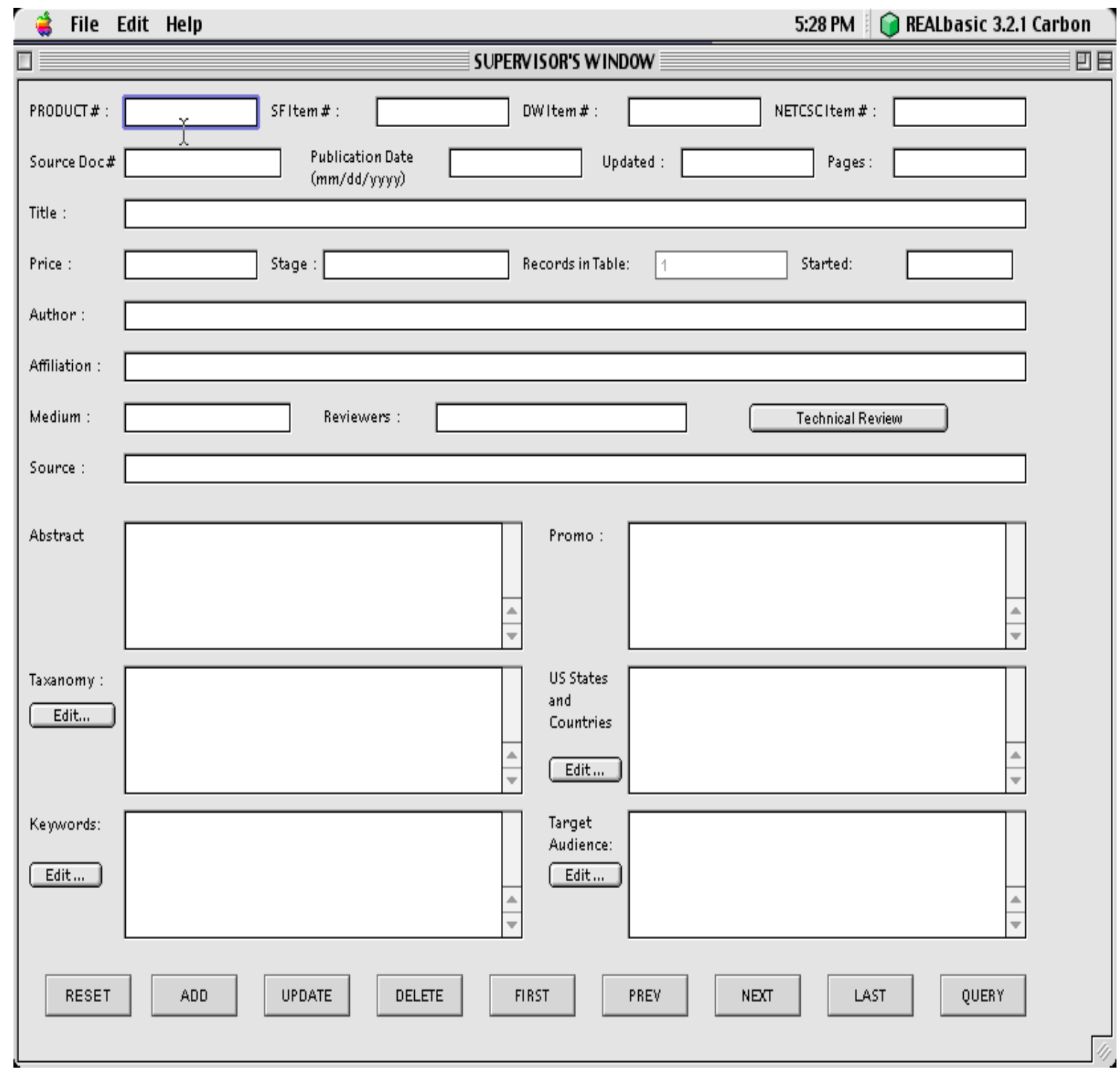

Figure 3.24 First Record in the Database

Note that the Records in Table field is incremented by 1 which lets us know that the record was successfully added into the database. 
One more item is sent through the whole process and all the information about the item is entered as shown below.

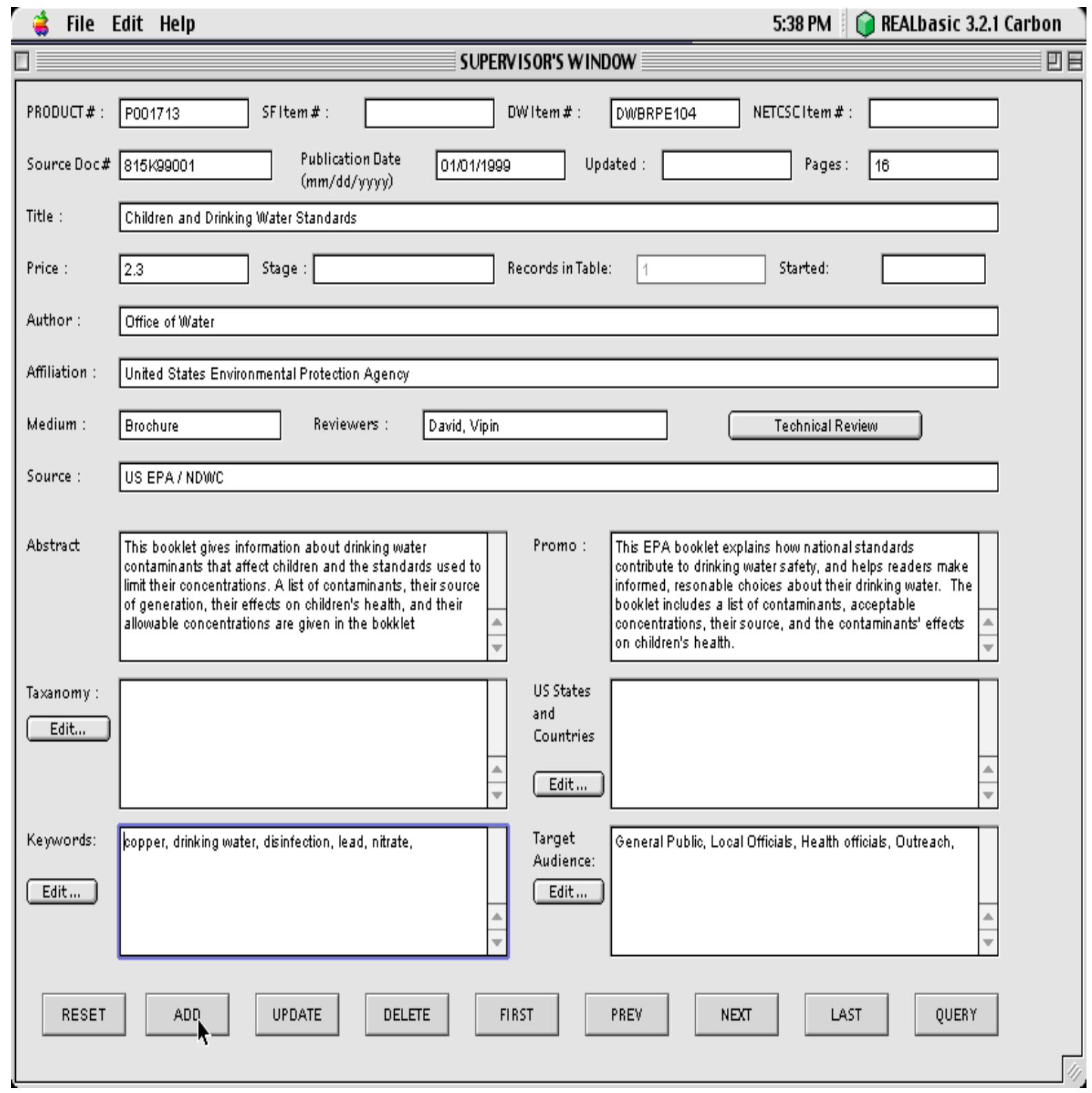

Figure 3.25: Information about Second Item

Upon clicking the ADD button, the current record is added and the Records in Table field is incremented by 1 as seen in Figure 3.26. 


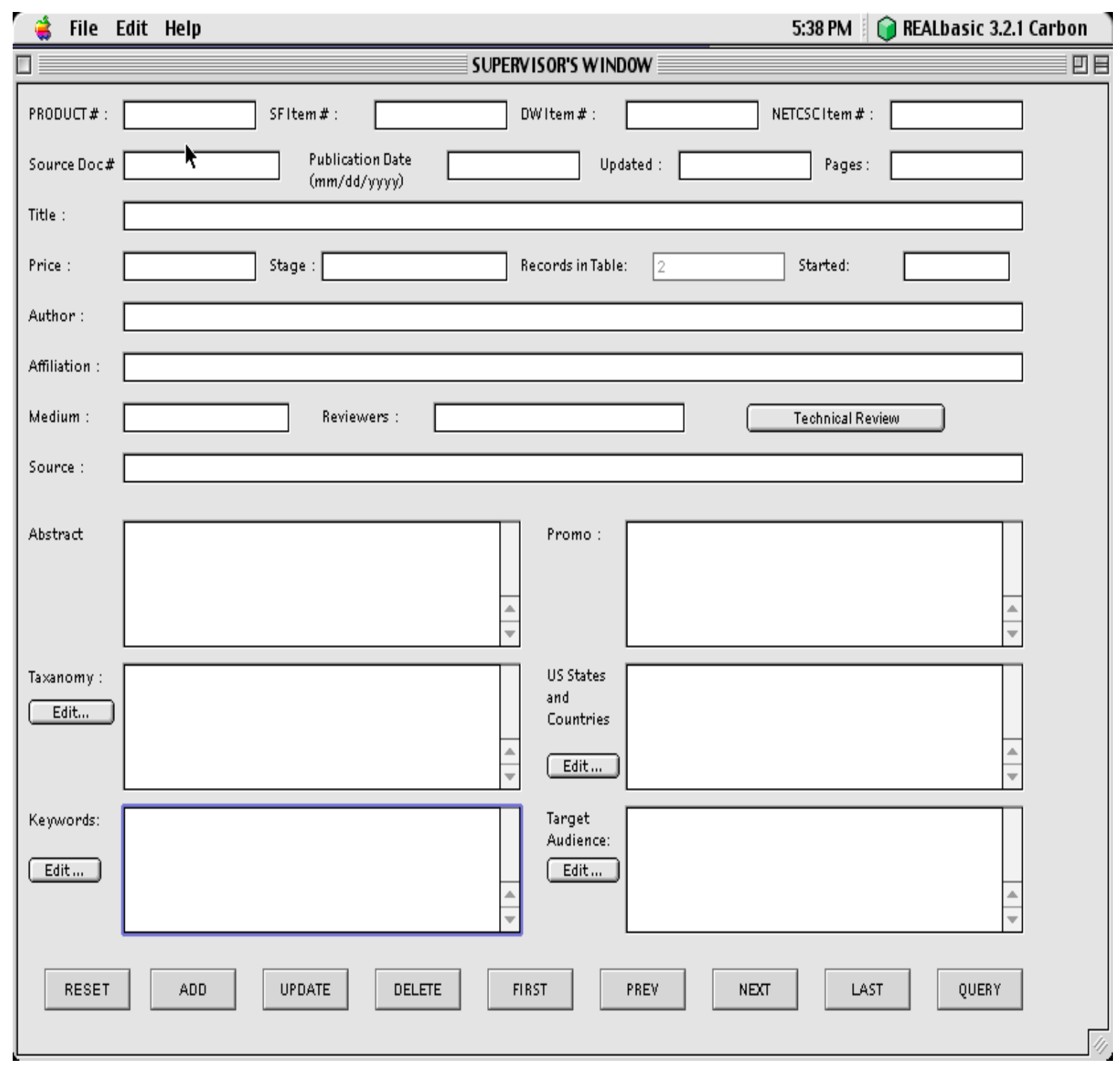

Figure 3.26: Second Record in the Database

To indicate the working features of buttons for other fields we add one more record into the database. A product number is assigned and few other pieces of information are fabricated for purpose of demonstration, as shown in Figure 3.27. 


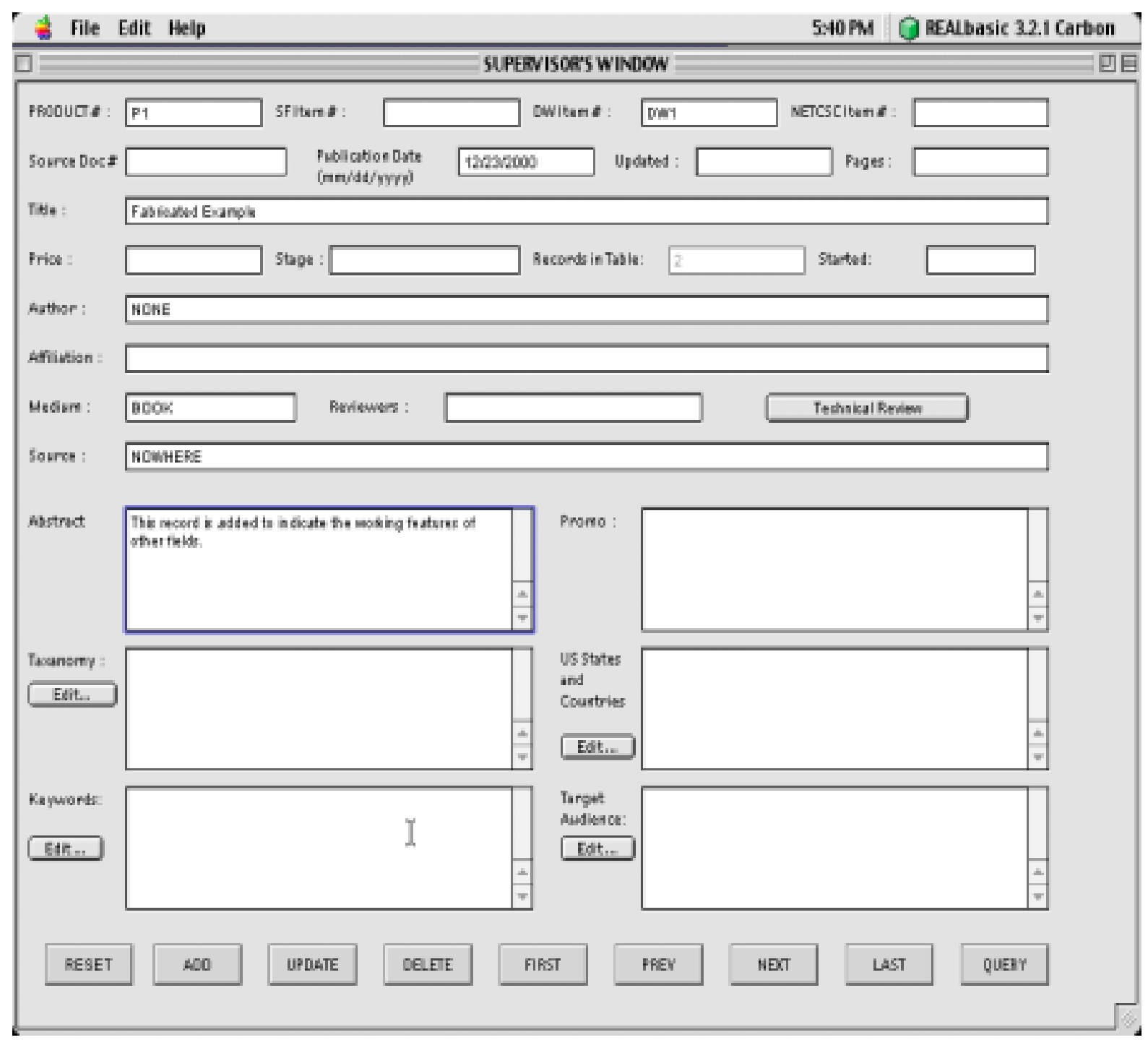

Figure 3.27: Information about one more record

Note that the above information is nowhere related to any item. The above record was just added to show how the buttons for other fields work.

Upon clicking the ADD button, the current record is added to the database and the Records in Table is incremented by 1 as shown from Figure 3.28. 


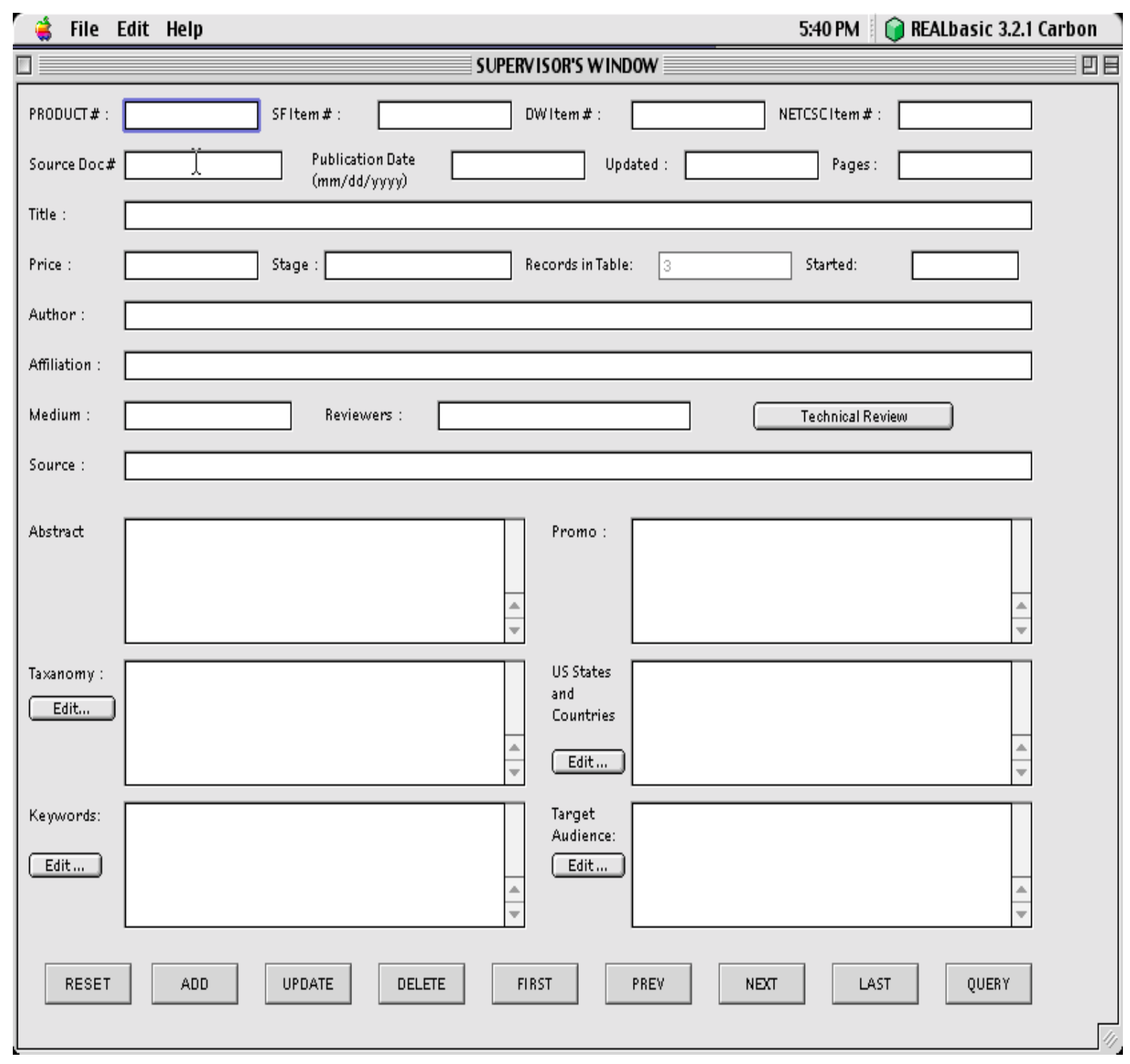

Figure 3.28: Third Record into Database

Note that at this stage we have 3 records in the database.

Clicking on the FIRST button pulls up the first record in the database as shown in Figure

\subsection{9 below.}




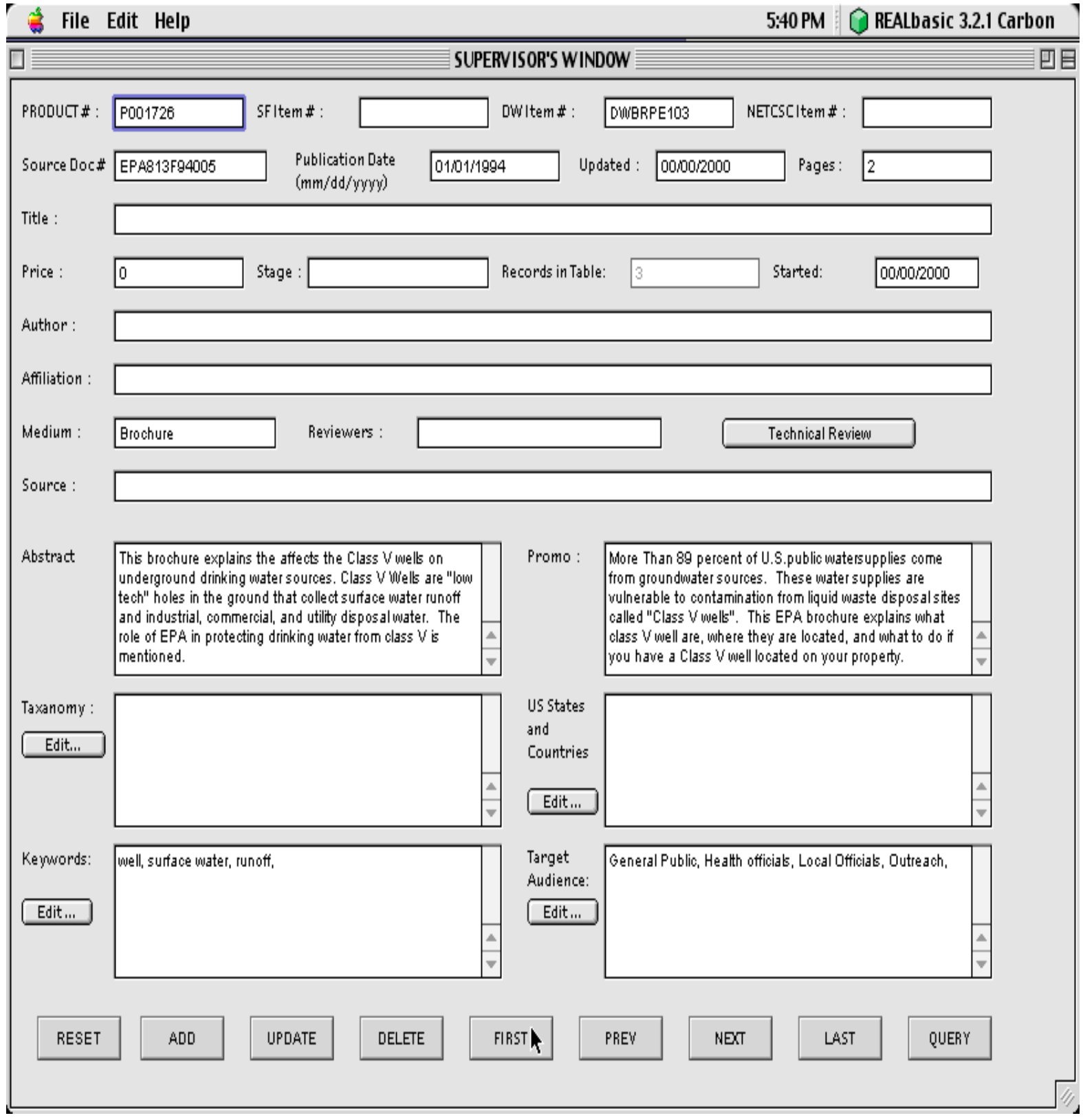

Figure 3.29: Window Indicating First Record

Clicking on the NEXT button pulls up the next record as shown in Figure 3.30. Clicking on the PREV button pulls up the previous record as seen from Figure 3.31. Clicking on the LAST button pulls up the last record in the database as seen from Figure 3.32. 


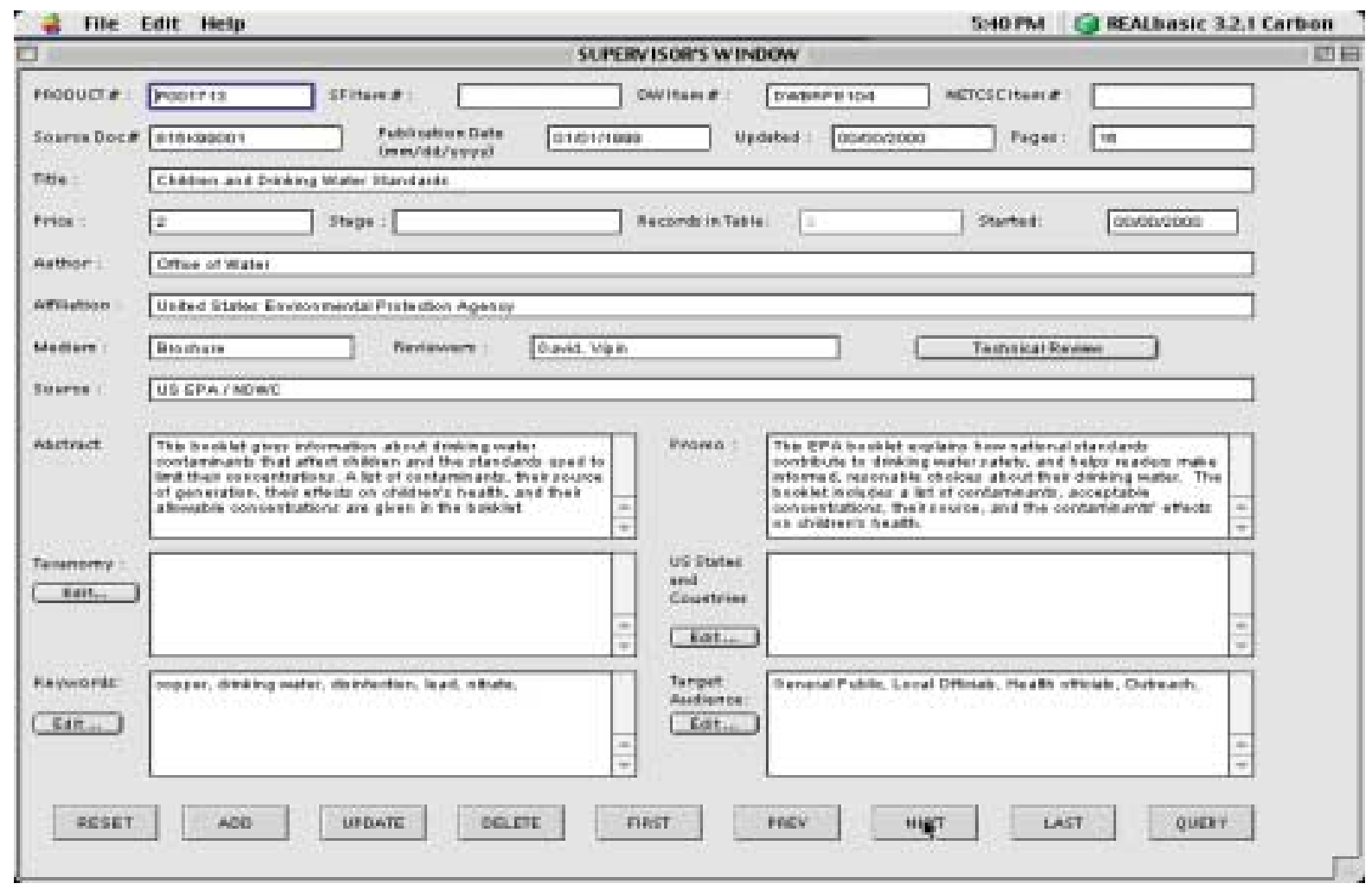

Figure 3.30: Window Indicating next Record

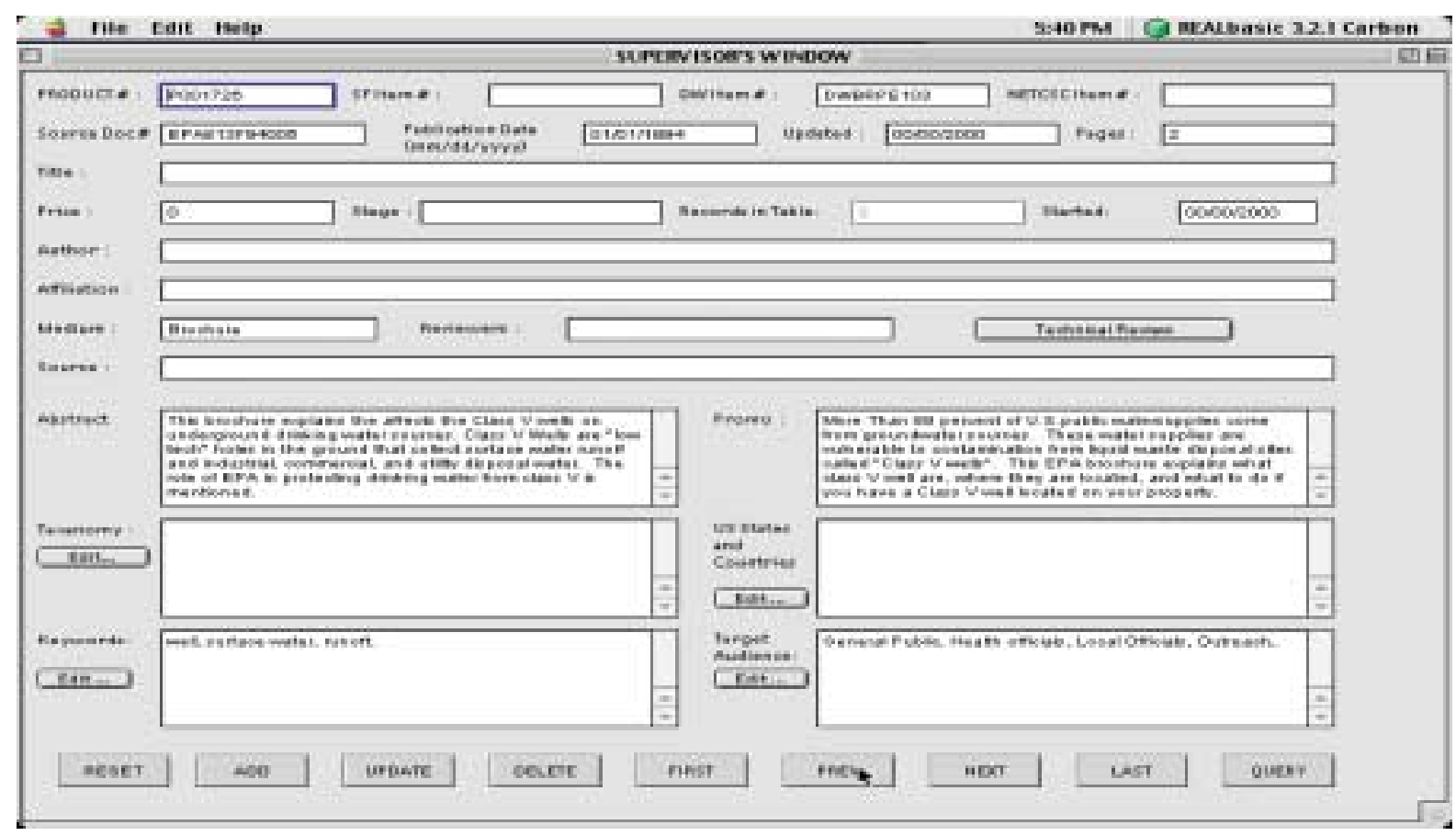

Figure 3.31: Window Indicating Previous Record 


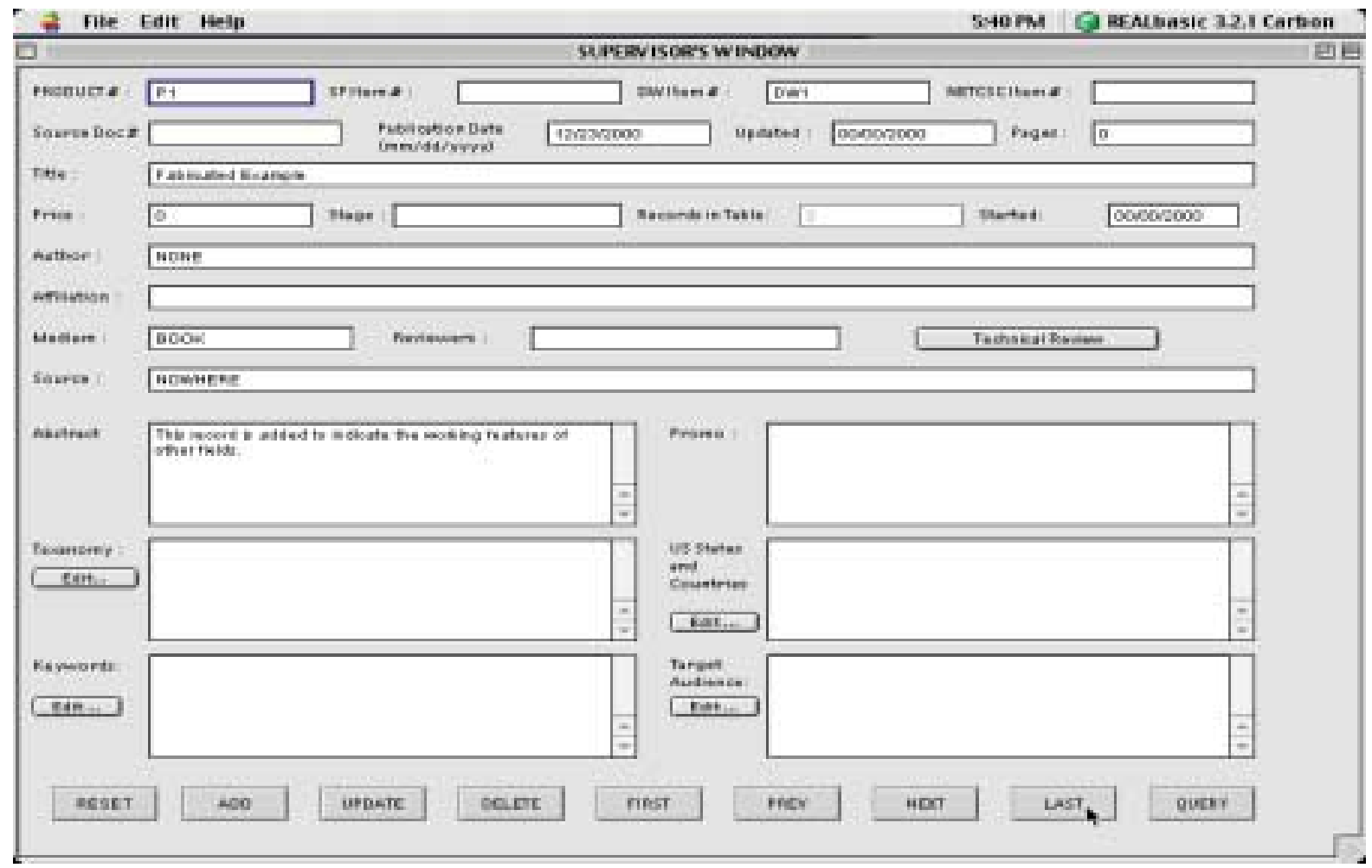

Figure 3.32: Window Indicating Last Record

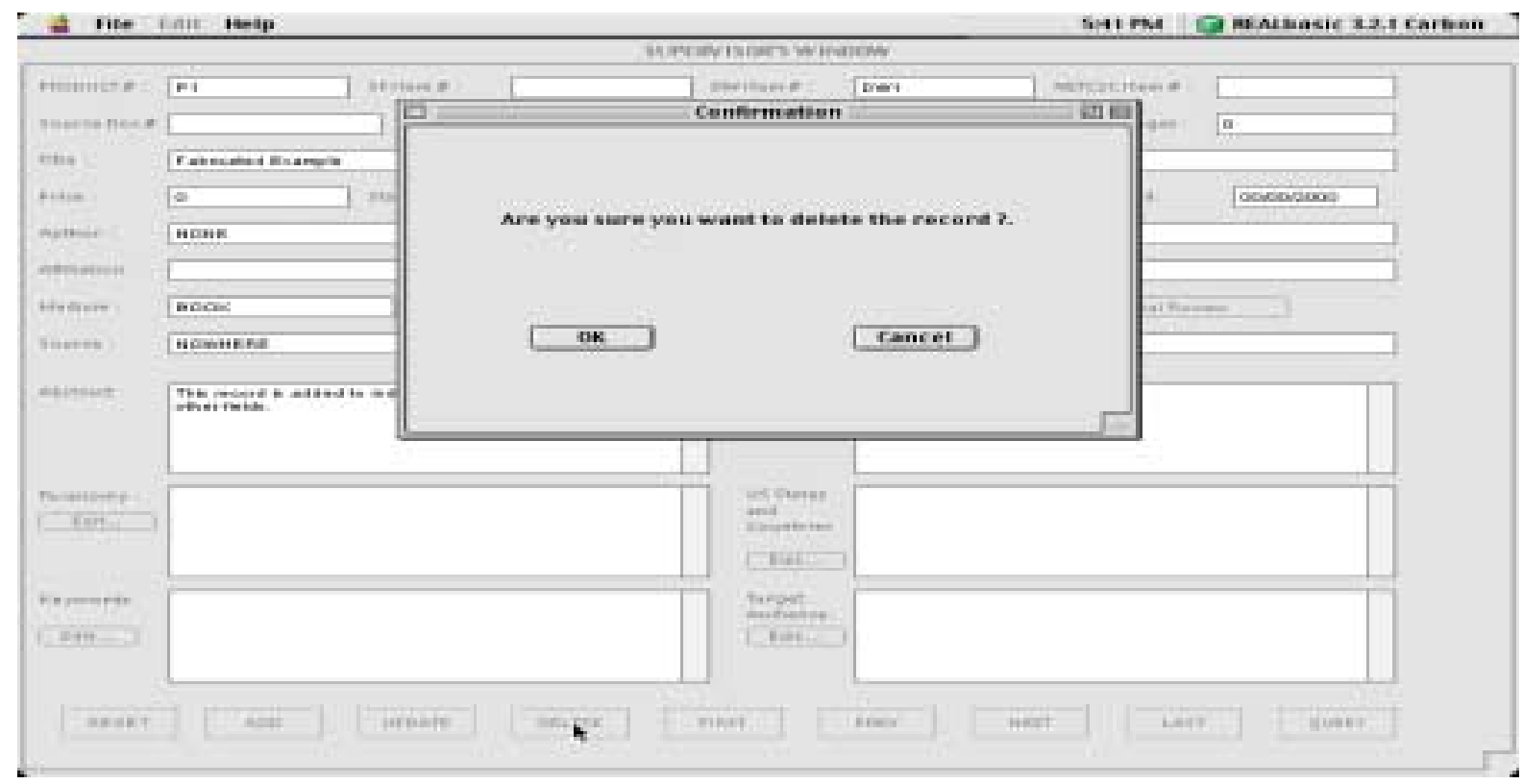

Figure 3.33: Confirmation Window 
Clicking on the DELETE button pulls up a confirmation window as seen from Figure 3.33 . Pressing the "OK" button on the confirmation window deletes the current record and the number of records in table is decremented by 1 as seen Figure 3.34. Pressing the Cancel button cancels the operation and the user is returned back to the main screen.

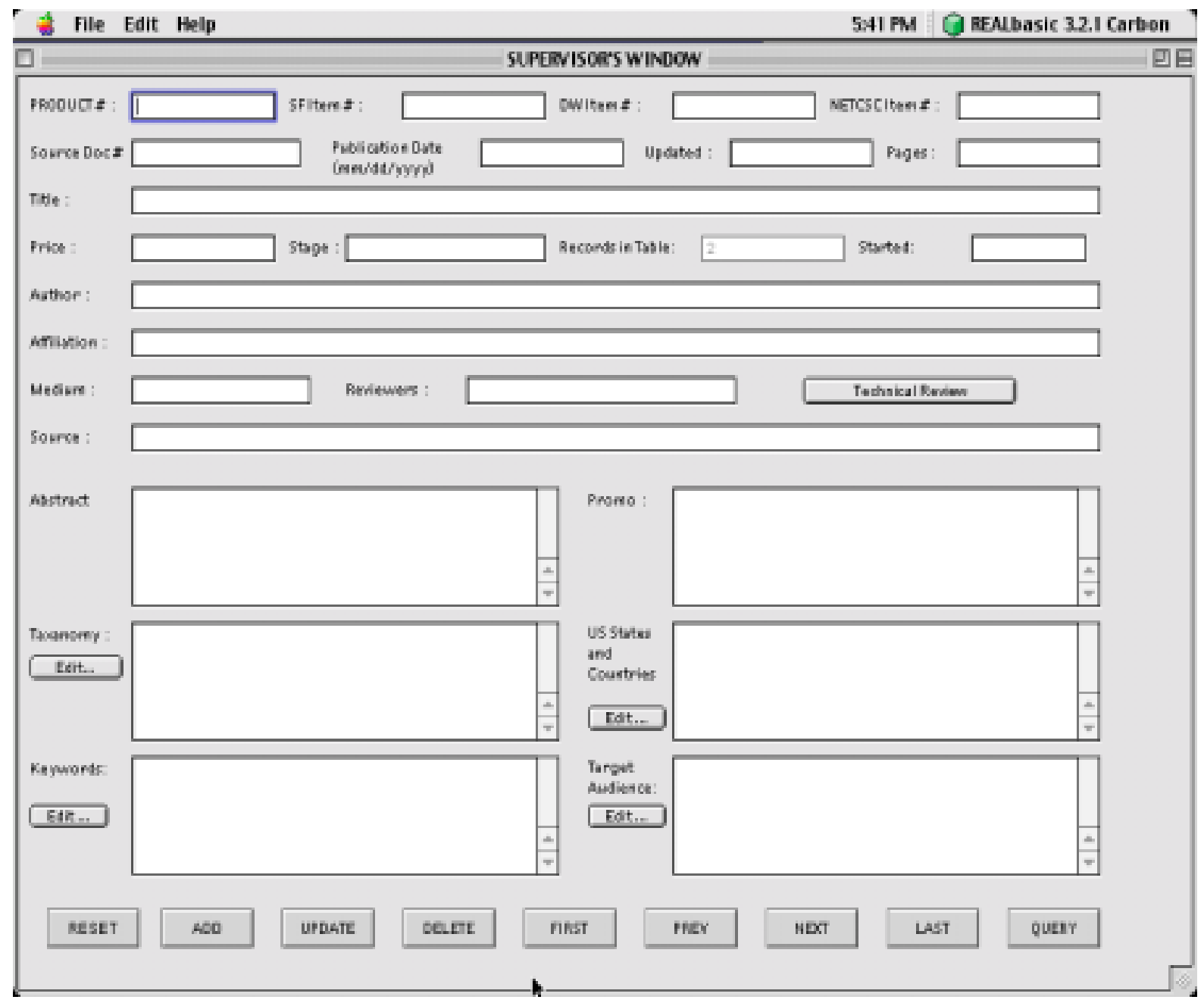

Figure 3.34: Delete Record 
To open the database click on File $\rightarrow$ Open

Choose the database as shown in Figure 3.35 and click on Open.

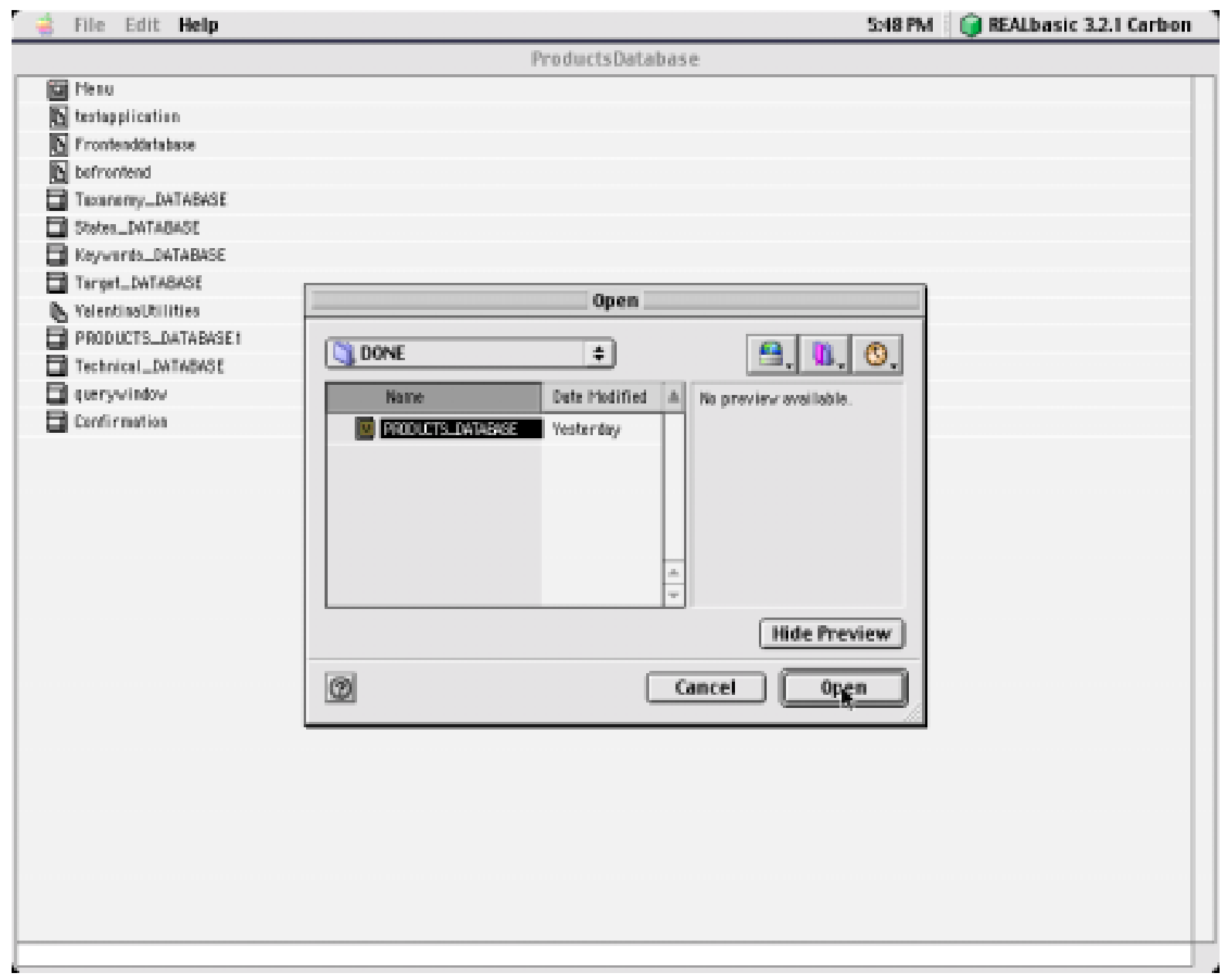

Figure 3.35: Open Database Window

This database contains 2 records as shown in Figure 3.36 and Figure 3.37. To do a search on the database click on the QUERY button. Clicking on the Query button takes us to the screen as seen from Figure 3.38. 


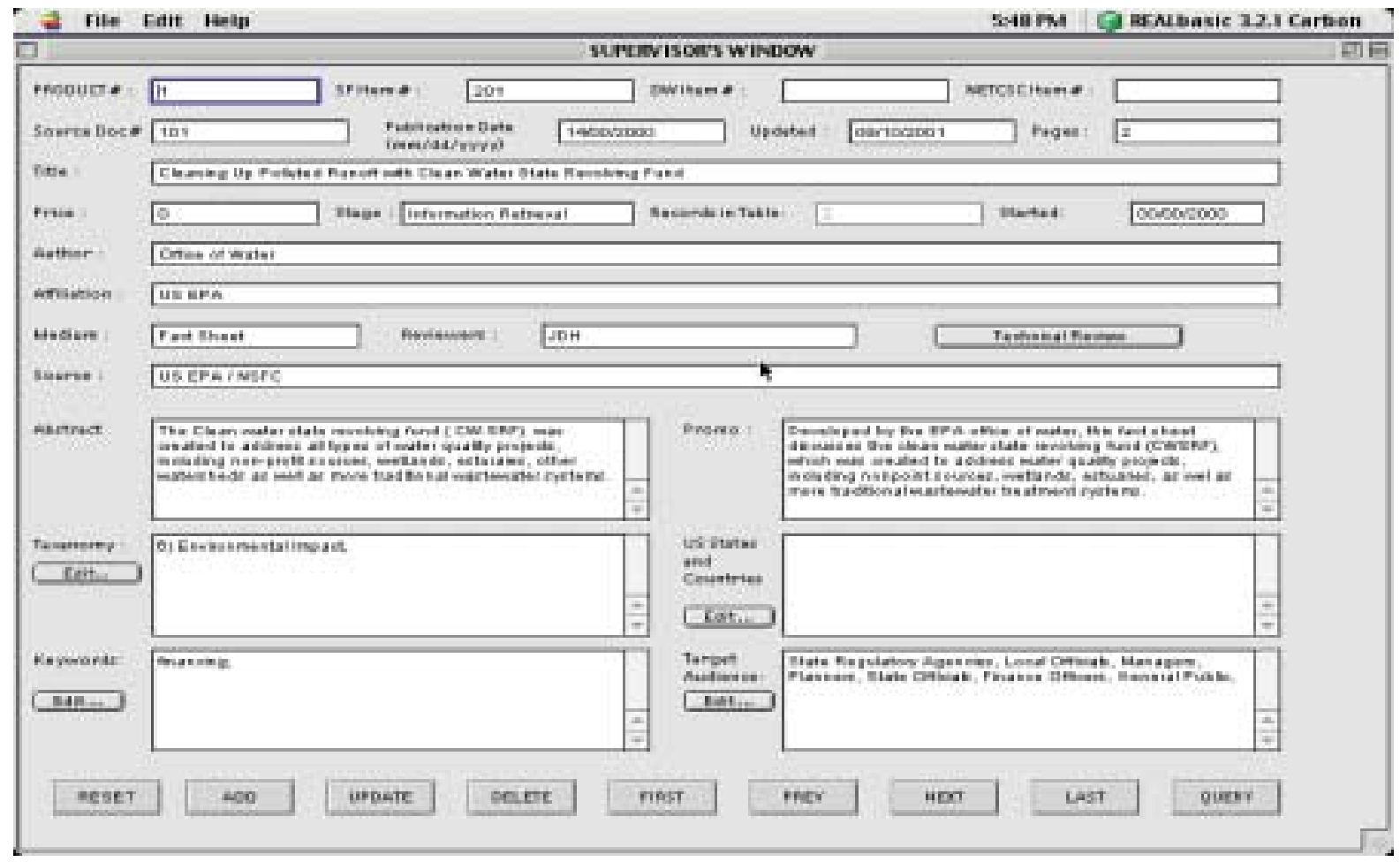

Figure 3.36: Record One of Products Database

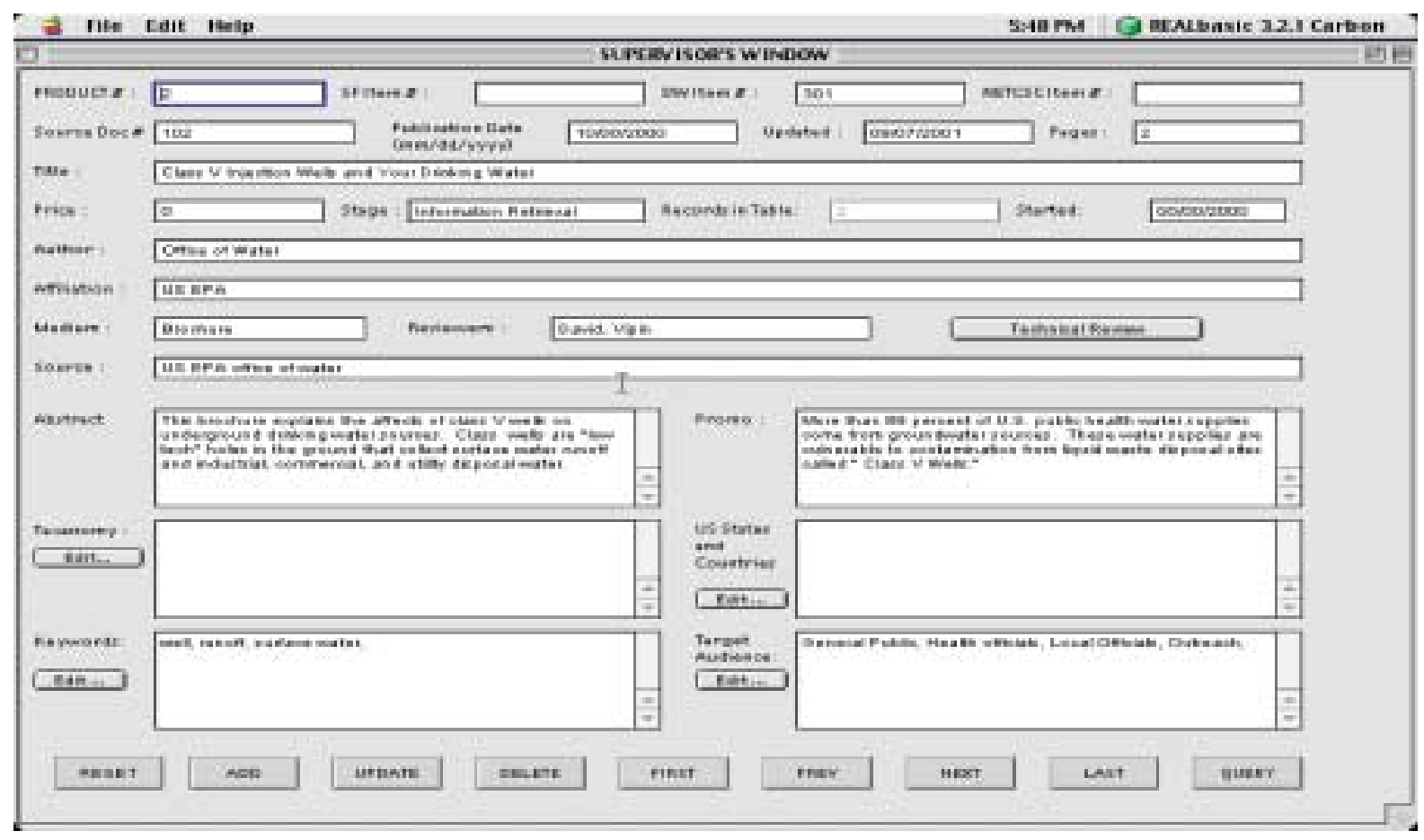

Figure 3.37: Record two of Products Database 


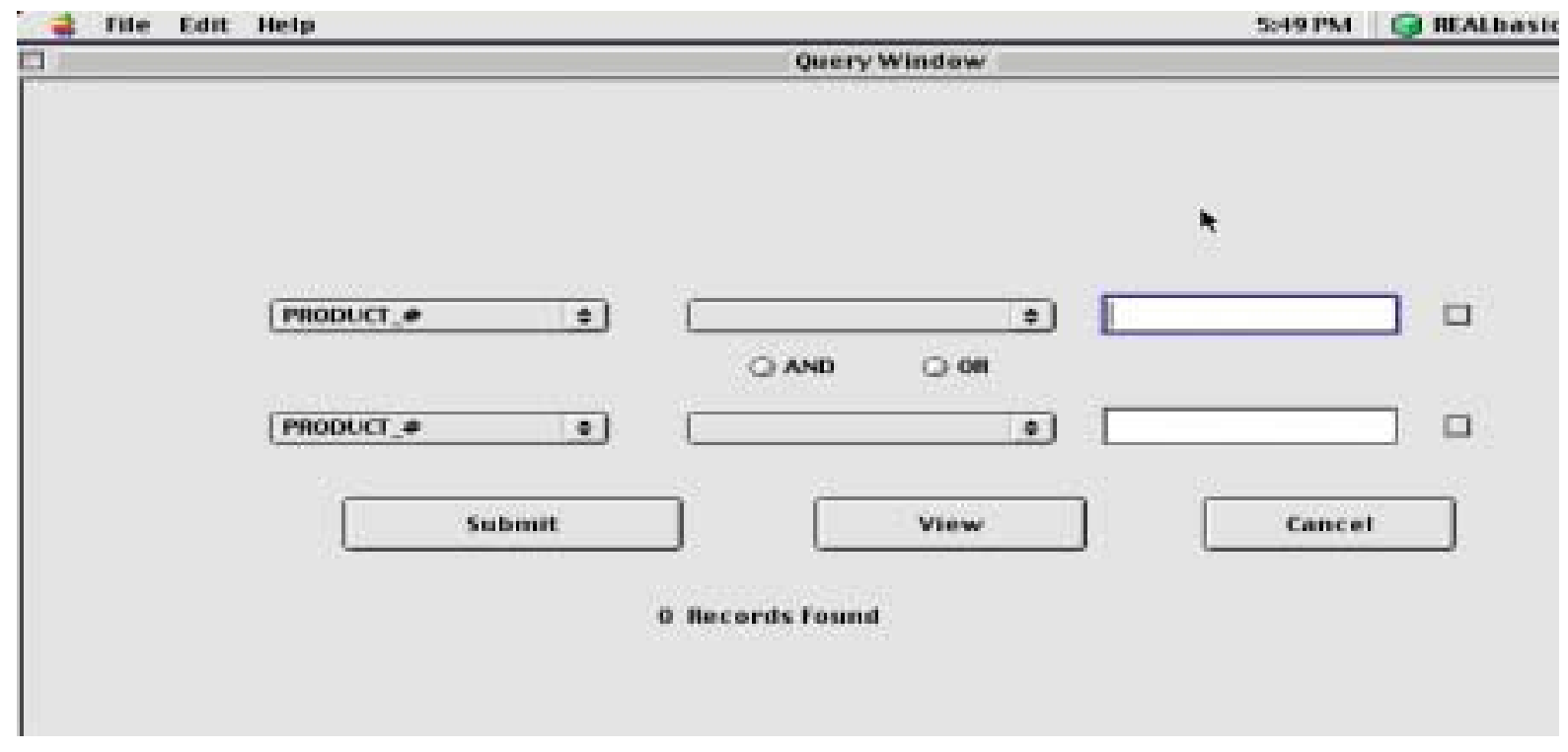

Figure 3.38: Query Window

Let us say we need to search by Product Number equal to 1. We choose Product _\# from popup menu 1, Equal to from popup menu 2, and type in 1 in the text box and click on the checkbox as seen from Figure 3.39. Clicking on Submit button pulls up one record as seen from Figure 3.40. Figure 3.41 shows the result when either Product Number is equal to 1 or 2

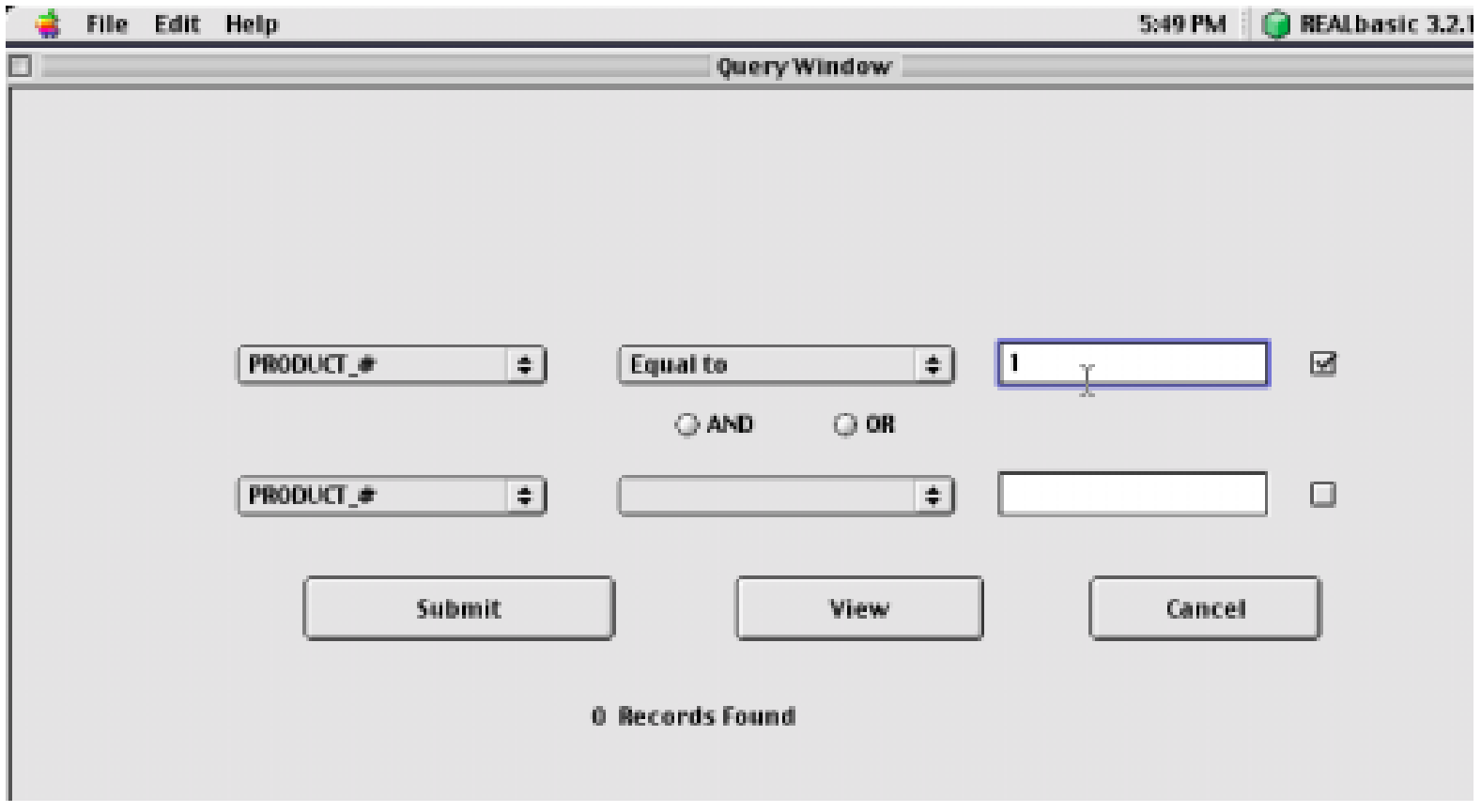

Figure 3.39: Query Entry Window 


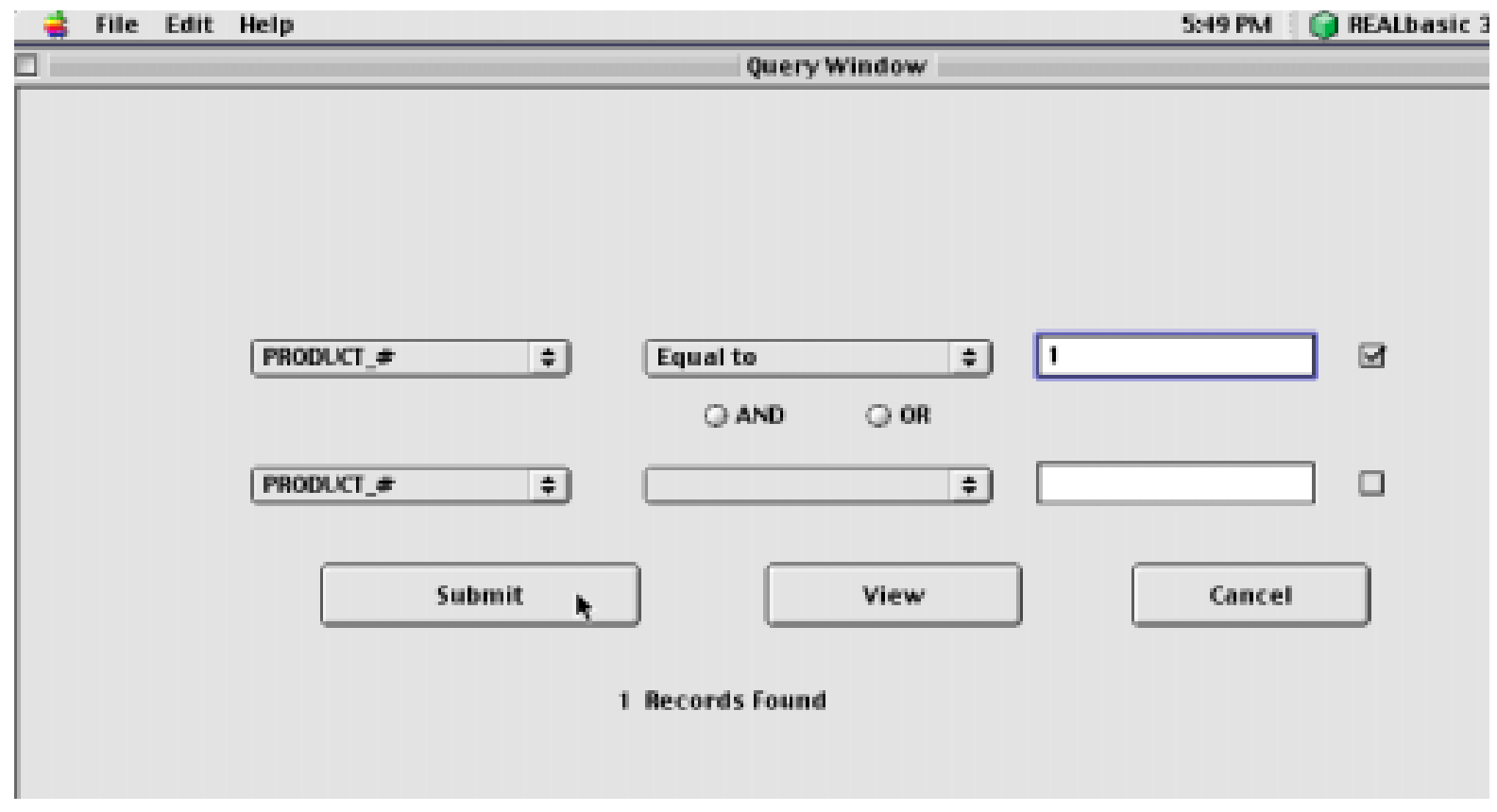

Figure 3.40: Query Submit Window

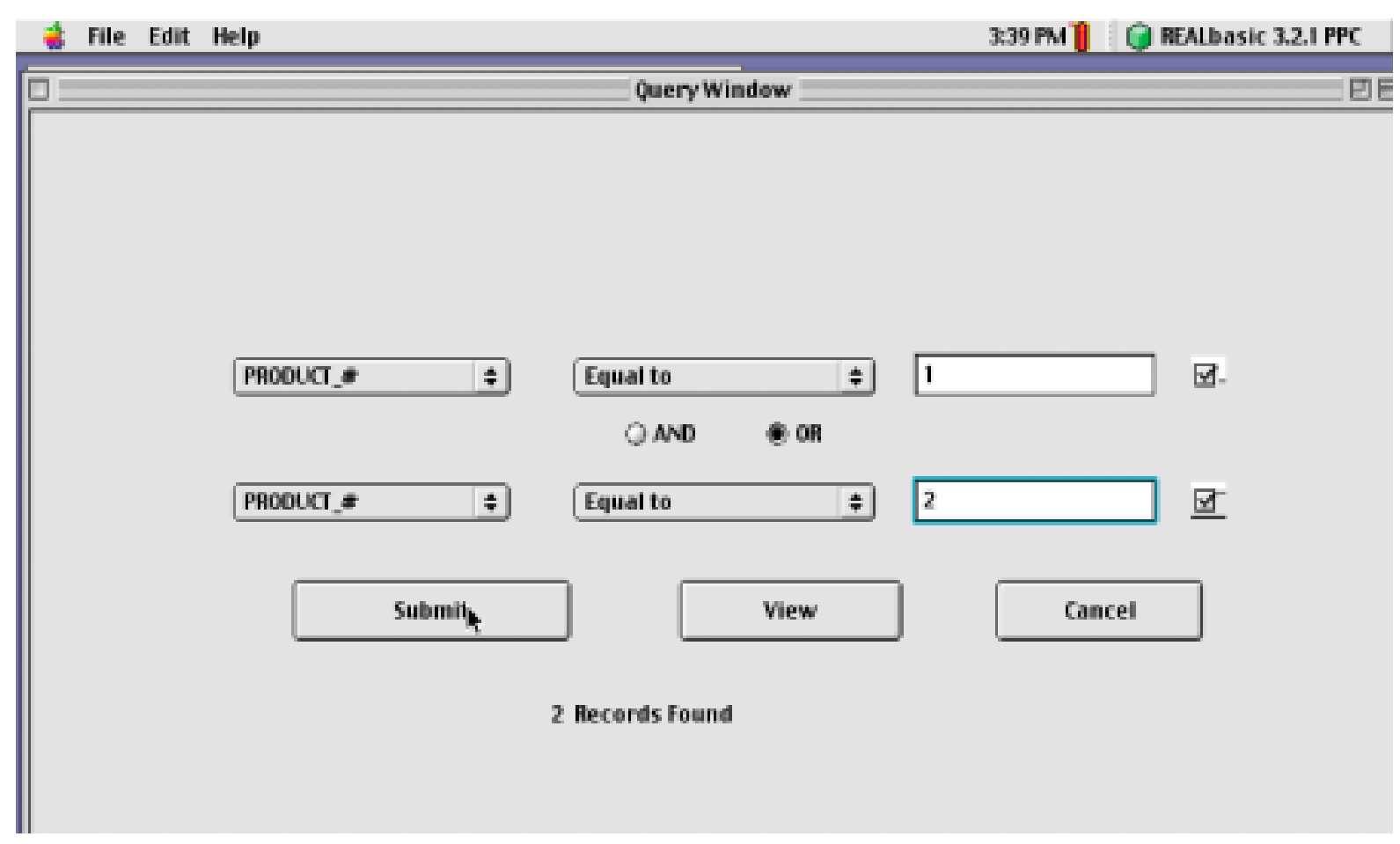

Figure 3.41: Second query Submit Window 
Clicking on the View button pulls up the screen below as seen from Figure 3.42. Note that it indicates there are 2 records and it is displaying the first record. The "FIRST", "NEXT", "LAST", and "PREV" buttons can be used to navigate between the records pulled up the query.

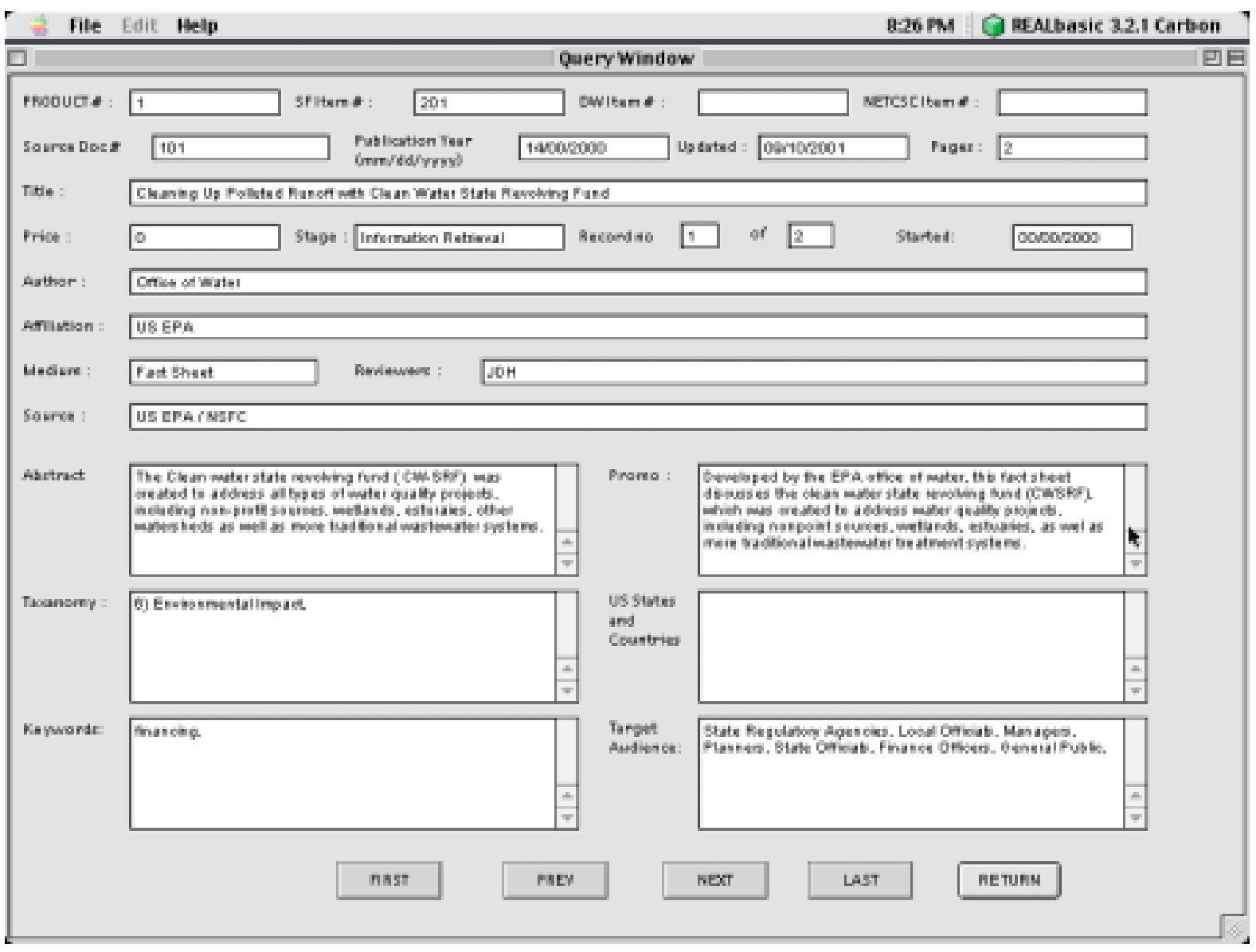

Figure 3.42: Query Result Window

Clicking on the Return button takes us back to the query screen as seen in Figure 3.43.

Clicking on the Cancel button on the query window takes us back to the screen as seen in

Figure 3.44. 


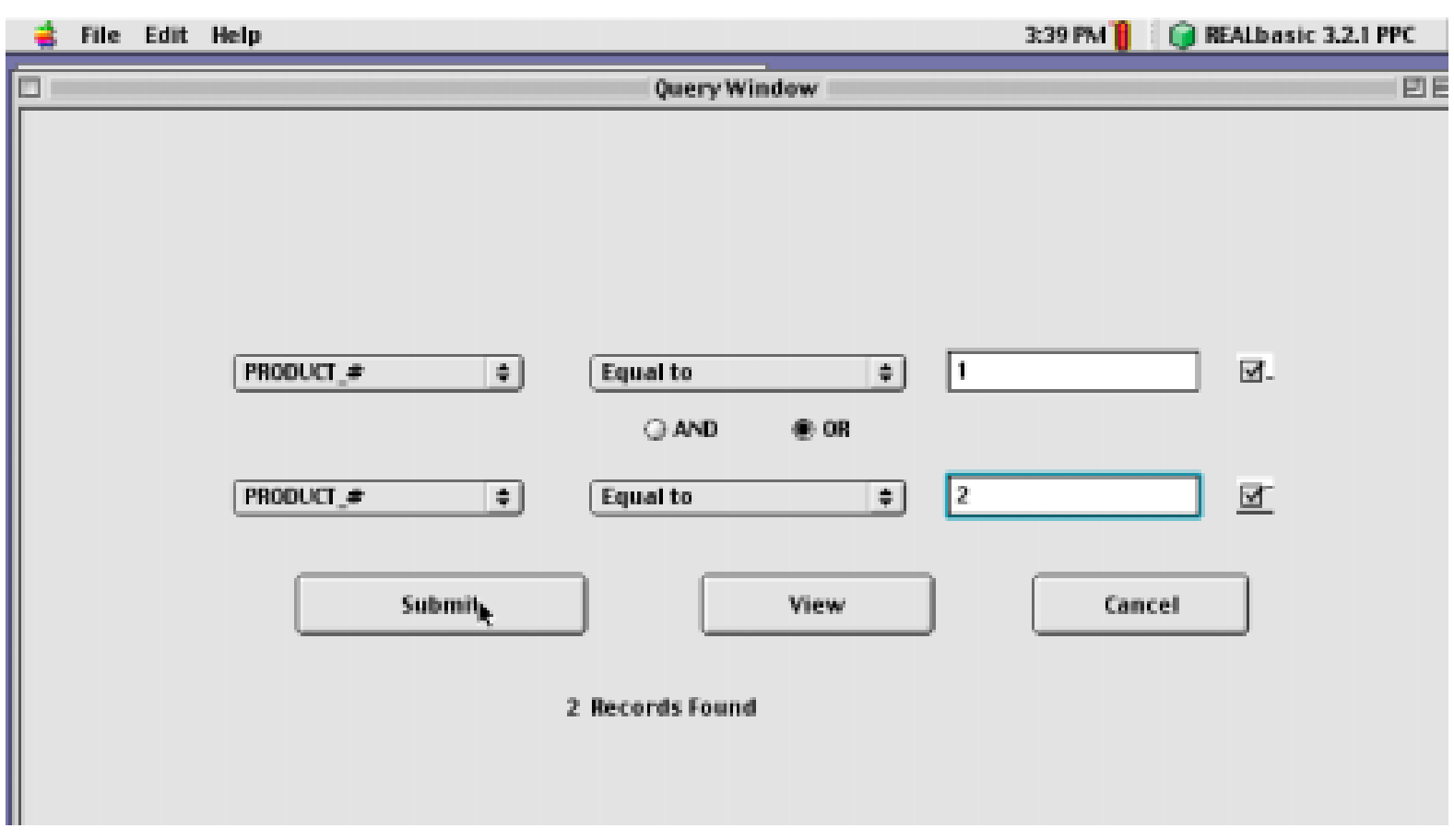

Figure 3.43: Final Query Submit window

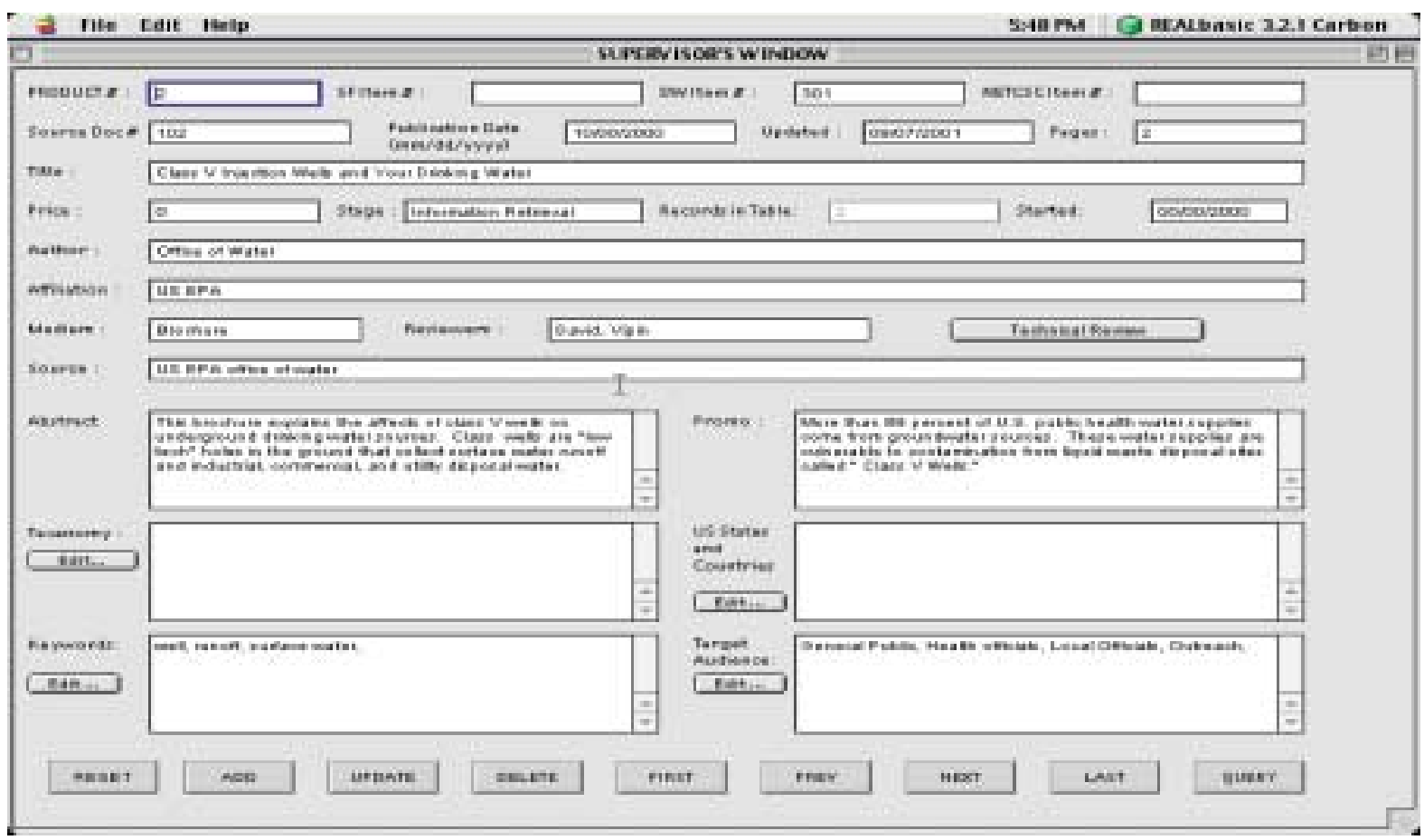

Figure 3.44: Products Database Front End. 


\section{CHAPTER 4: CONCLUSIONS/CONTRIBUTIONS}

\subsection{Timeline of Old Process}

The figure below shows the time taken by an item to go through the current "old" Product Review Process. The time indicates working days and is an average of time taken by an item to pass through the 3 programs.

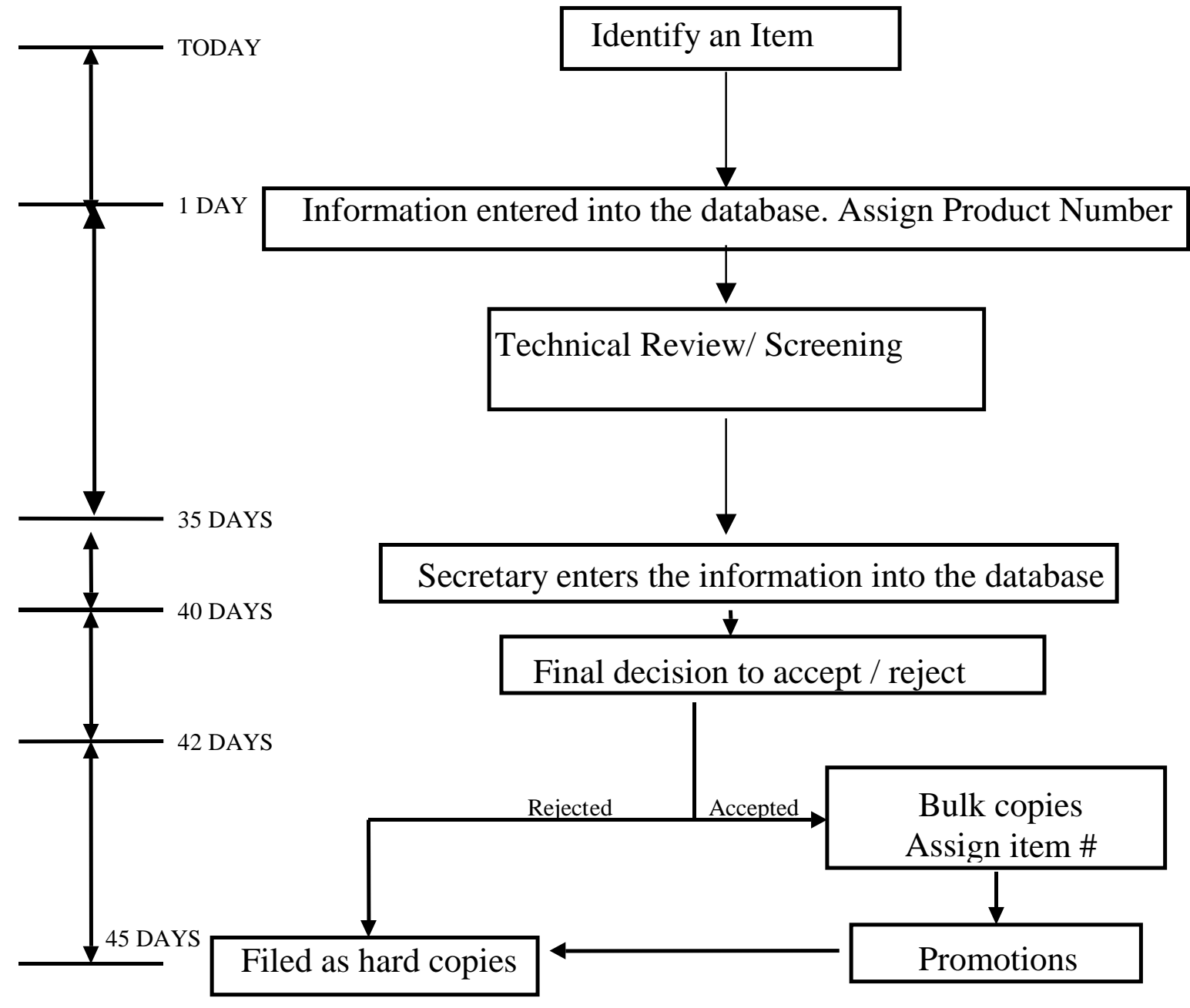

Figure 4.1: Timeline of OLD Process. 
Once the item is identified, the supervisor checks to see if the item is already present in the database. The supervisor then enters all the basic information of an item such as product number, EPA document number, on a buck sheet. This takes approximately 1 working day. Once all the basic information is entered into the buck sheet, the supervisor then passes the buck sheet with a copy of the item to the technical review stage. The supervisor makes sure that the item is sent to the relevant NESC Program. The technical reviewers wait till 5 or 6 items are ready for review. The technical reviewers review the item and based upon some criteria they decide to either accept or reject the item. They enter all the necessary information such as item number, abstract, keywords, on a buck sheet. Different NESC Programs perform this stage differently, that is, one NESC Program depends on one technical reviewer's decision to either accept or reject an item, while an other program has written criteria on which this decision is based. The other major flaw accruing during this stage is loss of the copy of the item while passing among various processing offices.

Once all the information is filled in on the buck sheet, the sheet is passed to the supervisor for data entry into the database. It usually would have taken atleast 30 - 35 days on an average before the supervisor gets the buck sheet with all the information entered. At this stage, the supervisor contacts the owners of the item to find out if the relevant NESC program can use it as a product. Once the final decision to either accept or reject the item is made, the supervisor enters all the information that is present in the buck sheet into the database. The supervisor usually requires approximately $5-7$ days to perform this stage. Note that the supervisor is solely responsible to enter all the information for all the items that are coming from all the Programs. The supervisor obtains bulk copies of items that are accepted. A copy of the accepted item is sent to the promotional specialist for a promotional abstract to be written. Finally the hard copy of all the items are stored in file cabinets. Thus marks the end of the product review process. On an average it would have taken an item 45 days to pass through the entire review process which is a long period. We now present the timeline for the proposed process. 


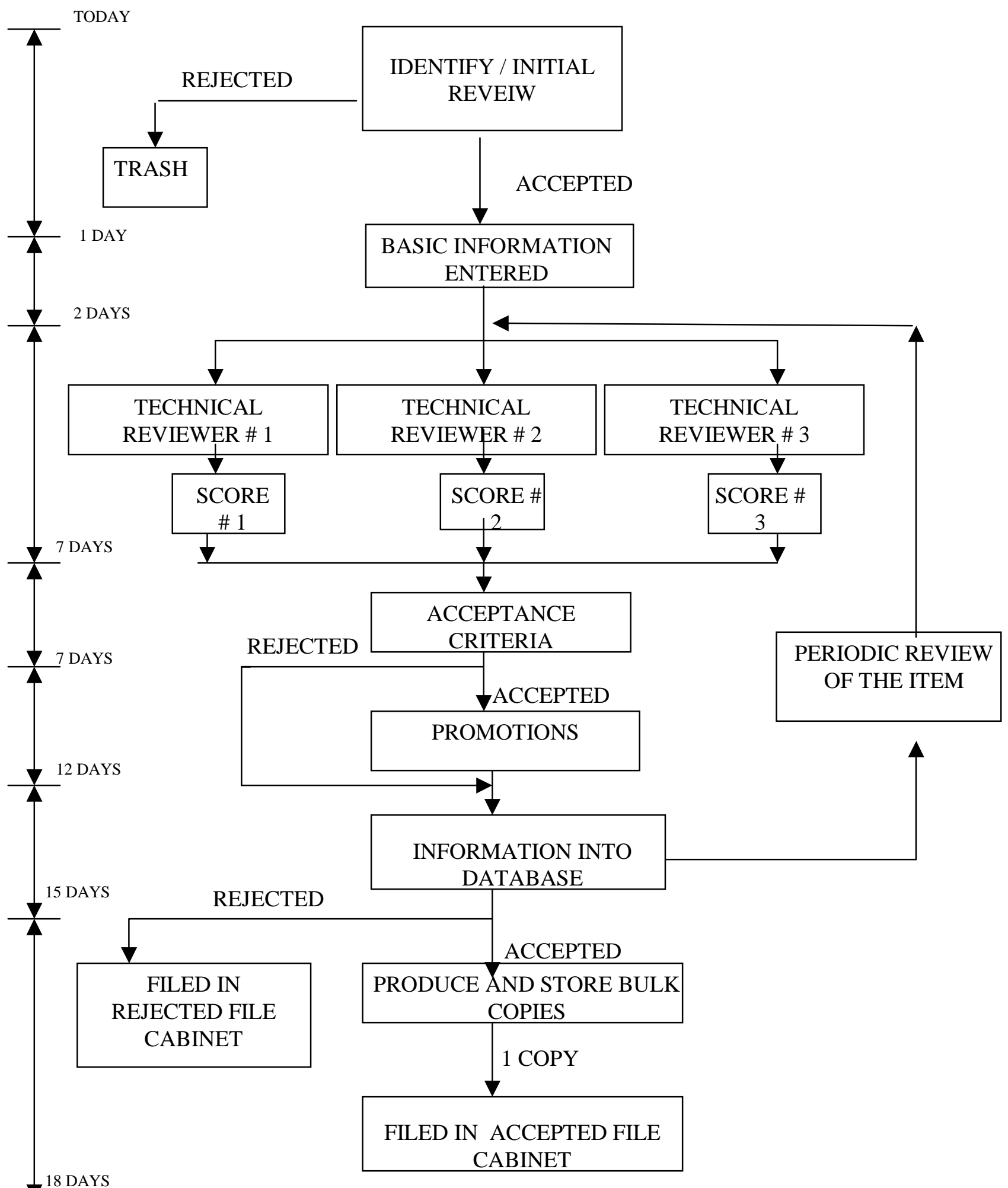

Figure 4.2: Time line of Proposed Process 


\subsection{Time line of Proposed Process}

The Figure 4.2 shows the time taken by the same item to pass through the proposed process. Once the item is identified, the supervisor checks the database to find out if the item already exists. Once the item passes the initial review, the supervisor contacts the owner of the item to find out if the relevant program can eventually use it as a product. For items that are accepted after the initial review, the supervisor enters the basic information into the database. It requires approximately 2 days on an average to reach this stage. Once all the basic information is entered, the supervisor sends the item for technical review. Note that just a copy of the item is sent, without a buck sheet. Once the technical reviewer is notified that an item is waiting to be reviewed, he/she enters the database and clicks on the Technical Review button. It takes the technical reviewer to a screen that has 5 questions. Depending on the relevant criteria, the technical reviewer scores the item on a scale of 1 - 10 and clicks on the submit button thus finishing one stage of the review. The item is sent to the second and third reviewer for the item to be reviewed and they also score the item. Once the item is reviewed by the technical reviewers and upon clicking on a done button, a message box pops up which lets the technical reviewer know whether the item is accepted or rejected using some predefined acceptance criteria. For items that are accepted, an item number is given and all the necessary information about the item is directly entered into the database. Then the item is sent to the promotions specialist for a promotional abstract to be written. This stage may require approximately $10-12$ days. Since no sheet is physically passed around, there is a lower possibility of any information loss.

Once the promotional abstract is written the item is sent back to the supervisor. The supervisor checks the database for errors and has the final authority to save the item into the database. The supervisor also notifies when the item needs to be reviewed again, periodically. This stage would require an average of approximately 3 - 4 days. For items that are rejected, the supervisor files the hard copies in a file cabinet for rejected items, by the product number.

For items that are accepted, the supervisor obtains get bulk copies and files a hard copy of the accepted item in the file cabinet designated for accepted item. This marks the end of product review process. On an average the proposed process is estimated to require 18 days for an item to go through the whole process. 


\subsection{Advantages of the proposed process over old process}

The overall process time would be reduced by half. Since information about when the whole process starts is known, the organization would have a clear picture of how long it took for them to identify the item. No time is spent on reviewing an item that cannot be used by an NESC program to be offered as a product. The technical review process would be streamlined. The technical review stage does not have to depend on the availability of only one reviewing person. A set of guidelines is initially decided upon, and every person reviewing the item, uses these guidelines to rate the item. With the use of predefined acceptance criteria, the item is either accepted or rejected. So, the review is not biased on a single individual's decision. Promotional abstract is written for accepted items soon after technical review is complete which smoothes the flow of work. Since all the information is entered directly into the database, no paper needs to be passed around during the whole process. This results in the reduction of information loss and process time. The supervisor checks the information entered in the database for errors before saving it to the database, thus reducing the number of typing errors present. The hard copies of accepted versus rejected items are easily accessible since the items are filed in different cabinets. Since the time for the next review of an item is known, the information about the item will not become obsolete or outdated.

\subsection{Contributions}

The current "Product Review Process" was studied in detail. Using the "Process Analysis Technique", NESC's needs were researched and a more efficient and effective process was developed which is expected to result in reduction of overall process time, information loss, etc. The technical review stage in the process is computerized and standardized, thus drastically reducing the time taken by an item to pass this stage. A database was developed to house all the information using RealBasic as the front end and Valentine as the backend. A corresponding search engine was also developed which is expected to result in easy retrieval of information. 


\subsection{Future Work}

- Drop down menus

- Medium ( Book, Brochure, Video, Tape, etc.)

- Document Source ( National Education Association, US EPA, US OSHA, etc.)

- Document Number.

- An automated system alerting the supervisor of periodic review.

- More search criteria.

- An optional text field for a technical reviewers to enter information about an item and the reason for accepting/rejecting an item.

- Segregate the list of US States and Countries by states and countries.

- Import records from current database to the new database. 


\section{REFERENCES}

1. Bales, Robert Freed [1950] Interaction Process Analysis:Aa method for the study of small groups. Addison-Wesley press, Cambridge, Mass.

2. Berlo, David Kenneth [1960] The Process of Communication; An introduction to theory and practice. Holt, Rinehart and Winston, New York.

3. Davenport, Thomas H [1993] Process Innovation: Reengineering work through information technology. Harvard Business School Press, Boston, Mass.

4. Keen, Peter G. W [1997] The Process Edge: Creating value where it counts. Harvard Business School Press, Boston, Mass.

5. Manne, Alan; Sussmann, Ed [1963] Studies in Process Analysis. Wiley, New York.

6. Melan, Eugene H. [1993] Process Management: Methods for improving products and services. McGraw-Hill, New York.

7. Montgomery, Henry; Svenson, Ola [1989] Process and Structure in Human Decision Making. Wiley, New York.

8. Murill, Paul W. [1981] Fundamentals of Process Control Theory. Instrument Society of America, Research Triangle Park, N.C

9. Newman, William Herman [1972] The Process of Management: Concepts, behavior, and practice. Prentice-Hall, Engelwood Cliffs, N.J

10. Parker, John Cecil; Rubin, Louis J. [1966] Process as Content. Rand McNally, Chicago

11. Ramirez, W. Fred [1994] Process Control and Identification. Academic Press, Boston, Mass.

12. Schein, Edgor H. [1969] Process Consultation: Its role in organization development. Addison - Wesley pub. Co., Reading Mass.

13. Web Page, National Research Center for Coal and Energy. NRCCE HOME PAGE: http://www.nrcce.wvu.edu.

14. Web Page, National Research Center for Coal and Energy. Overview of NRCCE divisions and programs: http://www.nrcce.wvu.edu/programsmain.htm

15. Web Page, National Environmental Services Center, About NESC:

http://www.nesc.wvu.edu/nesc/nesc_about.htm 
16. Web Page, National Small Flow Clearing House. http://www.nesc.wvu.edu/nsfc/nsfc index.htm

17. Web Page, National Drinking Water Clearinghouse.

http://www.nesc.wvu.edu/ndwc/ndwc_index.htm

18. Web Page, National Environmental Training Center for Small Communities. http://www.nesc.wvu.edu/netcsc/netcsc_index.htm

19. Web Page, National Onsite Demonstration Program. http://www.nesc.wvu.edu/nodp/nodp_index.htm

20. Westerberg, Arthur W. [1979] Process Flow sheeting. Cambridge University Press, Cambridge, New York 


\section{APPENDIX}

\section{Test Application:}

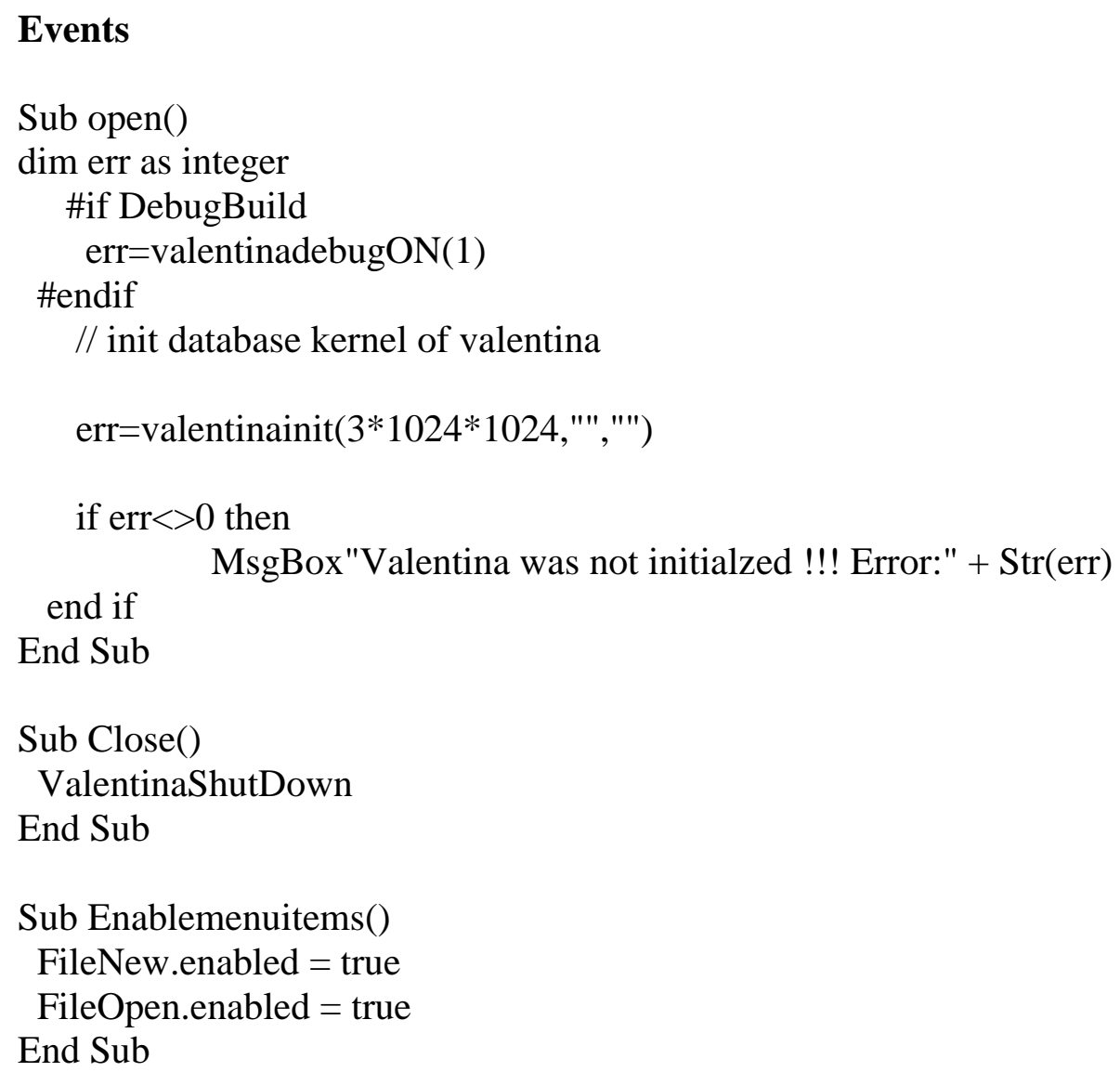

\section{Menu Handlers}

\section{File NEW:}

Sub Action as boolean

$\operatorname{dim} \mathrm{f}$ As folderItem dim w as PRODUCTS_DATABASE1

dim res as boolean

$\operatorname{dim}$ q As querywindow

$q=$ new querywindow

$\mathrm{f}=$ getSaveFolderItem("DB","Database1")

if $\mathrm{f}\langle>$ nil then $\mathrm{w}=$ new PRODUCTS_DATABASE1 


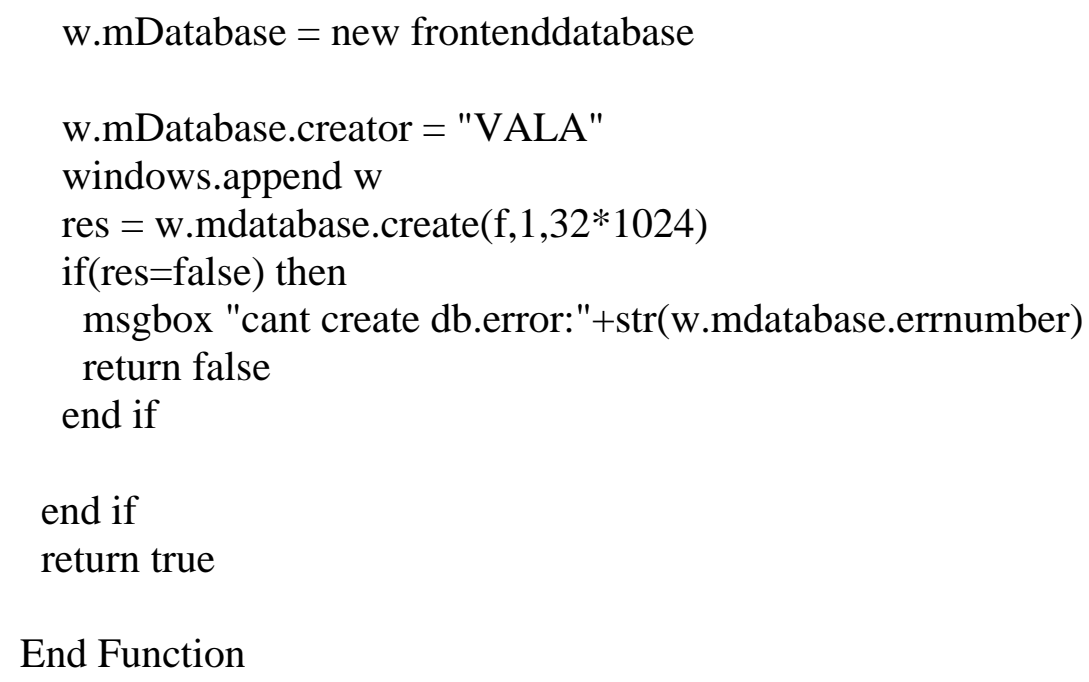




\section{FrontEndDatabase:}

\section{Properties}

mfrontend as bofrontend

\section{Methods}

mfrontend=new bofrontend

\section{bofrontend}

\section{Properties}

ProductNO as VString

SFNO as VString

DWNO as VString

NETCSCNO as VString

EPADOCNO as VString

Year as VDate

Updated as VDate

Pages as VUshort

Title as VVarchar

Price as VUlong

Stage as VString

DateStart as Vdate

Author as VVarchar

Affiliation as VVarchar

Medium as VString

Reviewers as VVarchar

Source as VVarchar

Abstract as Vtext

Promo as Vtext

Taxanomy as Vtext

States as Vtext

Keywords as Vtext

TargetAud as Vtext 


\section{Methods}

Bofrontend ( Method Name)

Sub bofrontend()

//to set the name of the BaseObject ( Table )

name $=$ "FRONTEND"

// to make the fields of the BaseObject ( Table )

ProductNO = new VString ( "Product_\#", 40, kV_Unique)

SFNO = new VString ( "SF_\#", 40, kV_Unique)

DWNO = new VString ( "DW_\#", 40, kV_Unique)

NETCSCNO $=$ new VString ( "NETCSC_\#", 40, kV_Unique)

EPADOCNO = new VString ( "EPADOC_\#", 50, kV_Nullable)

Year $=$ new VDate $($ "Year")

Updated = new VDate ( "Updated")

Pages = new VUshort ("Pages", kV_Nullable )

Title $=$ new VVarchar $($ "Title", 200$)$

Price $=$ new VUlong $($ "Price")

Stage $=$ new VString $($ "Stage", 60$)$

DateStart $=$ new Vdate $($ "DateStart")

Author = new VVarchar ( "Author", 100 )

Affiliation = new VVarchar $($ "Affiliation", 100$)$

Medium = new VString ( "Medium", 50)

Reviewers = new VVarchar ( "Reviewers", 100)

Source $=$ new VVarchar $($ "Source", 50)

Abstract $=$ new Vtext $($ "Abstract", $3 * 1024)$

Promo $=$ new Vtext $($ "Promo", 3*1024 )

Taxanomy = new Vtext ( "Taxanomy",3*1024)

States $=$ new Vtext $($ "States", $3 * 1024)$

Keywords = new Vtext $($ "Keywords",3*1024 )

TargetAud $=$ new Vtext $($ "Targetaud",3*1024 )

End Sub 


\section{PRODUCTS_DATABASE1:}

\section{Properties}

mDatabase as Frontenddatabase

\section{Methods}

Collectdb

Populatedb

Sub Collectdb()

if $($ mDatabase $=$ nil $)$ then return

end if

mdatabase $\cdot$ mfrontend.PRODUCTNO.value $=$ EditField1 $\cdot$ text mdatabase. mfrontend.SFNO.value $=$ EditField2.text mdatabase. $\mathrm{mfrontend}$.DWNO.value $=$ EditField 3 .text mdatabase. $m$ frontend.NETCSCNO.value $=$ EditField4 $\cdot$ text

mdatabase $\cdot$ mfrontend.EPADocNO.value $=$ EditField7 .text mdatabase.mfrontend.Year.Setstring(EditField8.text) mdatabase.mfrontend.Updated.SetString(EditField9.text) mdatabase. $m$ frontend.Pages.value $=\mathrm{Val}($ EditField10.text $)$

mdatabase. mfrontend. Title. value $=$ EditField11.text

mdatabase. mfrontend. Price. value $=$ Val $($ EditField12.text $)$

mdatabase. mfrontend .Stage .value $=$ Editfield22.text

mdatabase.mfrontend.DateStart.SetString(Editfield23.text)

mdatabase $\cdot$ mfrontend .Author .value $=$ EditField13.text

mdatabase. mfrontend.Affiliation $\cdot$ value $=$ EditField14.text

mdatabase $\cdot$ mfrontend.Medium $\cdot$ value $=$ EditField15.text

mdatabase. mfrontend $\cdot$ Reviewers.value $=$ EditField16.text

mdatabase $\cdot$ mfrontend .Source $\cdot$ value $=$ EditField17.text

mdatabase. mfrontend.Abstract.value $=$ EditField18. Text mdatabase. $m$ frontend.Promo.value $=$ EditField19.text mdatabase mfrontend .Taxanomy $\cdot$ value $=$ EditField20.text 
mdatabase. mfrontend. States.value $=$ EditField21.text mdatabase. mfrontend.keywords.value $=$ EditField5.text mdatabase. mfrontend .TargetAud.value $=$ EditField6.text

\section{End Sub}

Sub populatedb()

dim res as Boolean

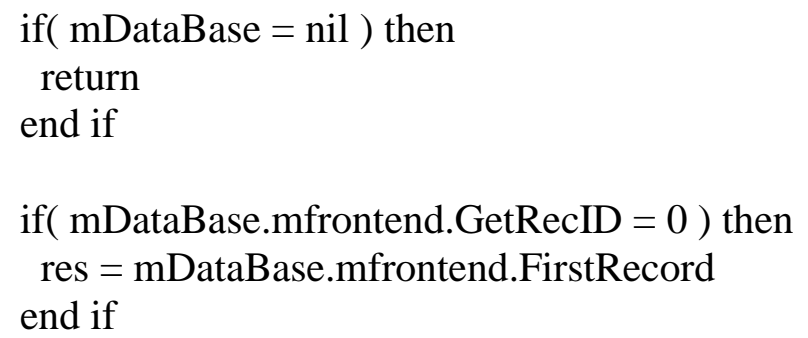

EditField11.text $=$ mdatabase. mfrontend .Title.value

EditField12.text $=$ Str $($ mdatabase. mfrontend.Price. value $)$

Editfield22.text $=$ mdatabase. $m$ frontend.Stage.value

Editfield23.text $=$ mdatabase. mfrontend.DateStart.getstring

EditField13.text $=$ mdatabase. mfrontend .Author.value

EditField14.text $=$ mdatabase $\cdot$ mfrontend. Affiliation. value

EditField15.text $=$ mdatabase. mfrontend.Medium.value

EditField16.text $=$ mdatabase. mfrontend .Reviewers.value

EditField17.text $=$ mdatabase. mfrontend .Source .value 
EditField18. Text =mdatabase. mfrontend.Abstract.value EditField19.text $=$ mdatabase. mfrontend.Promo.value EditField20.text $=$ mdatabase.mfrontend.Taxanomy.value EditField21.text $=$ mdatabase. mfrontend .States.value EditField5.text $=$ mdatabase. mfrontend. keywords.value EditField6.text $=$ mdatabase. mfrontend . TargetAud. value

\title{
End Sub
}

\section{Controls}

ADD
UPDATE
DELETE
FIRST
PREV
NEXT
LAST
QUERY
RESET
TechnicalReview
edit_Taxanomy
edit_states
edit_keywords
edit_Targetaudience

\author{
ADD \\ Sub Action() \\ mdatabase.mfrontend.SetBlank // Clear Memory Buffer \\ collectdb \\ // Read Data \\ mdatabase.mfrontend.AddRecord \\ populatedb \\ End Sub \\ UPDATE \\ Sub Action() \\ Collectdb \\ mdatabase.mfrontend.updateRecord \\ End Sub \\ DELETE \\ Sun Action() \\ dim res as boolean
}




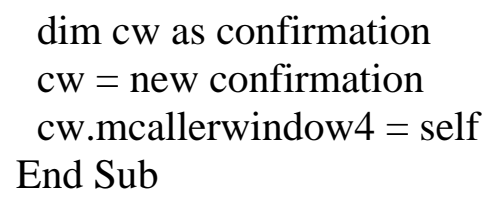

\section{FIRST}

Sub Action()

dim res as Boolean

res $=$ mDatabase.$m$ Frontend.FirstRecord

Populatedb

End Sub

PREV

Sub Action()

dim res as Boolean

res $=\mathrm{mDatabase} . \mathrm{mFrontend}$.Prevrecord

Populatedb

End Sub

NEXT

Sub Action()

dim res as Boolean

res $=$ mDatabase. $m$ Frontend.NextRecord

Populatedb

End Sub

\section{LAST}

Sub Action()

dim res as Boolean

res $=\mathrm{mDatabase} \cdot \mathrm{mFrontend}$.LasttRecord

Populatedb

End Sub

QUERY

Sub Action()

dim qw as querywindow

Hide

$\mathrm{qw}=$ new querywindow

qw. mcallerwindow $=$ self

End Sub

RESET

Sub Action() editField1.text $=$ "" 


$$
\begin{aligned}
& \text { editField2.text }=\text { "" } \\
& \text { editField3.text }=\text { "" } \\
& \text { editField4.text }=\text { "" } \\
& \text { editField5.text }=\text { "" } \\
& \text { editField6.text }=\text { "" } \\
& \text { editField7.text }=\text { "" } \\
& \text { editField8.text }=\text { "" } \\
& \text { editField9.text }=\text { "" } \\
& \text { editField10.text }=\text { "" } \\
& \text { editField11.text }=\text { "" } \\
& \text { editField12.text }=\text { "'" } \\
& \text { editField13.text }=\text { "" } \\
& \text { editField14.text }=\text { "" } \\
& \text { editField15.text }=\text { "" } \\
& \text { editField16.text }=\text { "" } \\
& \text { editField17.text }=\text { "" } \\
& \text { editField18.text }=\text { "" } \\
& \text { editField19.text }=\text { "'" } \\
& \text { editField20.text }=\text { "" } \\
& \text { editField21.text }=\text { "" } \\
& \text { editField22.text }=\text { "" } \\
& \text { editField23.text }=\text { "" }
\end{aligned}
$$

End Sub

TechnicalReview

Sub Action()

Technical_DATABASE.show

End Sub

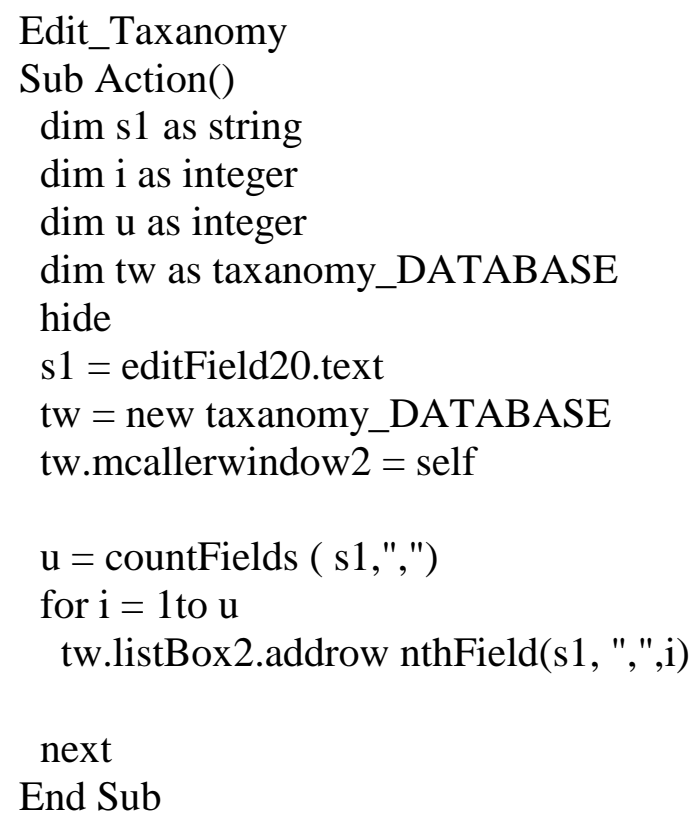

Edit_Taxanomy

Sub Action()

$\operatorname{dim} \mathrm{s} 1$ as string

$\operatorname{dim} \mathrm{i}$ as integer

$\operatorname{dim} u$ as integer

dim tw as taxanomy_DATABASE

hide

s1 = editField20.text

$\mathrm{tw}=$ new taxanomy_DATABASE

tw.mcallerwindow $2=$ self

$\mathrm{u}=$ countFields $(\mathrm{s} 1, \mathrm{,}, \mathrm{n})$

for $\mathrm{i}=1$ to $\mathrm{u}$

tw.listBox2.addrow nthField(s1, ",",i)

next

End Sub 


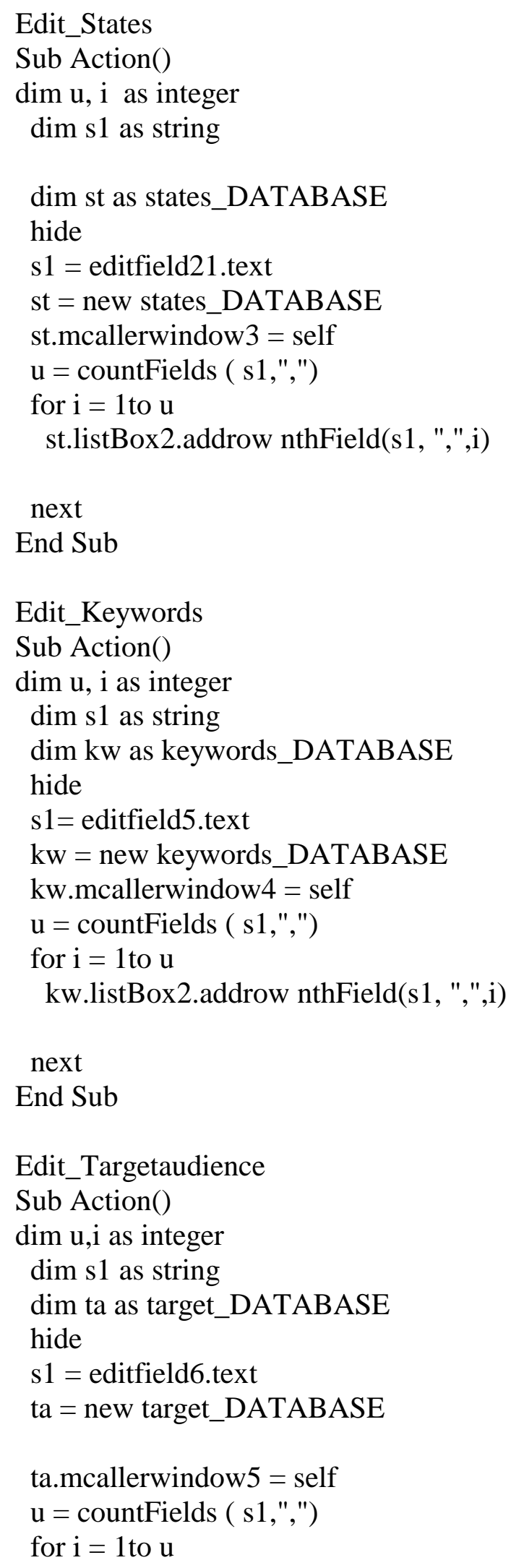


ta.listBox2.addrow nthField(s1, ",,,i)

next

End Sub

\section{TECHNICAL_DATABASE:}

\section{Controls}

SUBMIT

DONE

SUBMIT

Sub Action()

Dim a, b, c, d, e As Integer

Dim total, overall, no As Integer

Dim avg As Double

$$
\begin{aligned}
& \mathrm{a}=\mathrm{Val}(\text { editfield1.text) } \\
& \mathrm{b}=\mathrm{Val} \text { (EditField2.text) } \\
& \mathrm{c}=\mathrm{Val}(\text { EditField3.text) } \\
& \mathrm{d}=\mathrm{Val} \text { (EditField4.text) } \\
& \mathrm{e}=\mathrm{Val} \text { (EditField5.text) }
\end{aligned}
$$

total $=\mathrm{a}+\mathrm{b}+\mathrm{c}+\mathrm{d}+\mathrm{e}$

overall $=\operatorname{val}($ editfield6.text $)$

overall $=$ overall + total

editfield6.text $=\operatorname{str}($ overall $)$

no $=\operatorname{val}($ editfield $7 . t e x t)$

no $=$ no +1

editfield $7 . \operatorname{text}=\operatorname{str}($ no $)$

avg $=$ overall $/$ no

editfield8.text $=\operatorname{str}(\mathrm{avg})$

editfield1.text $="$ "

editfield2.text $="$ "

editfield3.text $=$ " "

editfield4.text $="$ "

editfield5.text $=$ " "

\section{End Sub}

DONE

Sub Action()

If val(editfield8.text) $>=40$ Then 
msgbox " The item under technical review is accepted "

Technical_DATABASE.close

Else

msgbox " The item under technical review is rejected because it does not pass the acceptance criteria "

Technical_DATABASE.close

End if

End Sub

\section{Taxanomy_DATABASE:}

\section{Properties}

Mcallerwindow2 as Products_Database1

\section{Controls}

ADD

DELETE

DONE

CANCEL

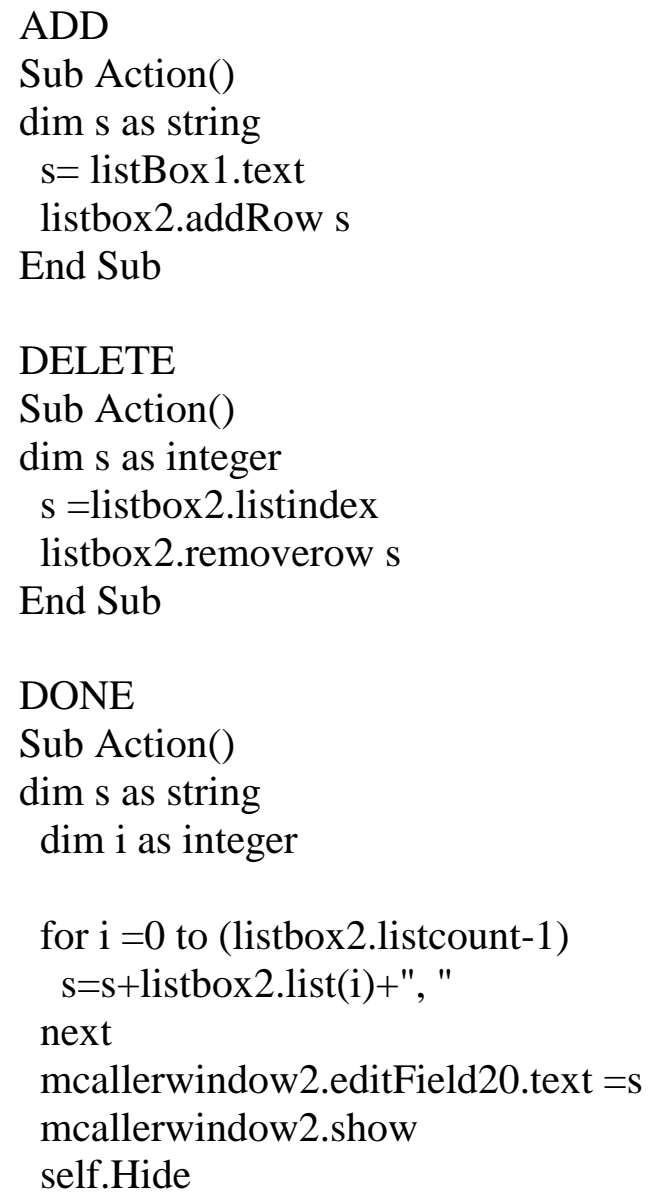




\section{End Sub}

\section{CANCEL}

Sub Action()

self.hide

mcallerwindow2.show

End Sub

\section{States_DATABASE:}

\section{Properties}

Mcallerwindow3 as Produicts_Database1

\section{Controls}

ADD

DELETE

DONE

CANCEl

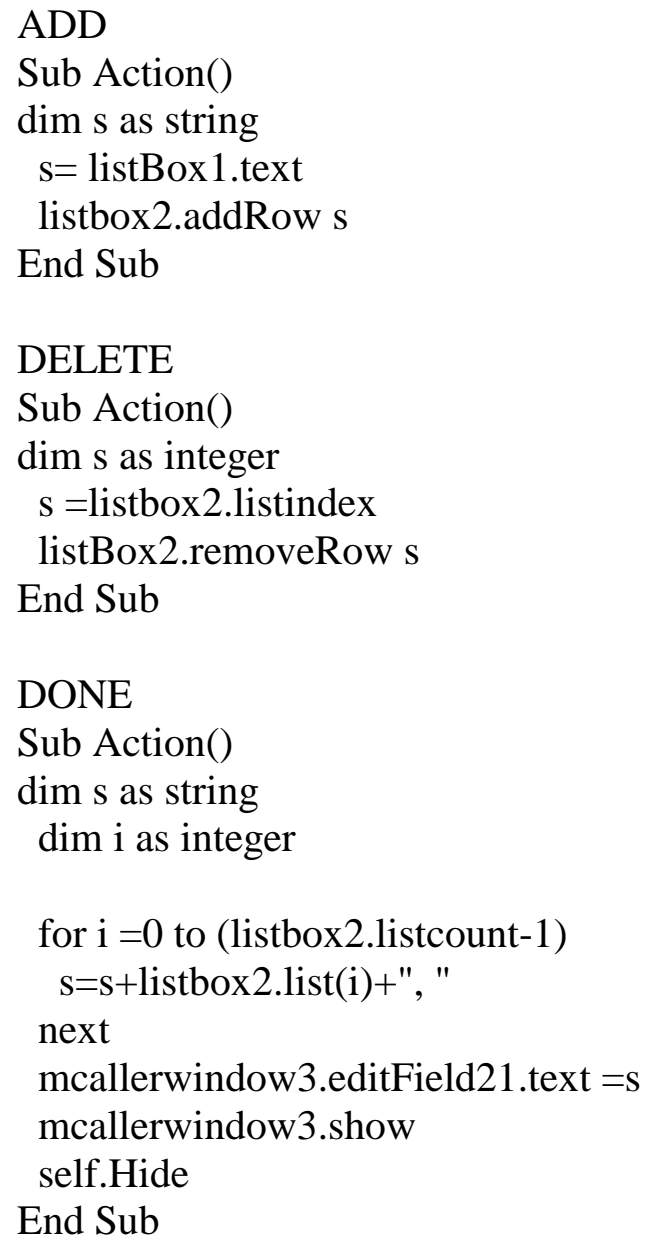


CANCEL

Sub Action()

self.hide

mcallerwindow3.show

EndSub

\section{Keywords_DATABASE:}

\section{Properties}

Mcallerwindow4 as Produicts_Database1

\section{Controls}

ADD

DELETE

DONE

CANCEL

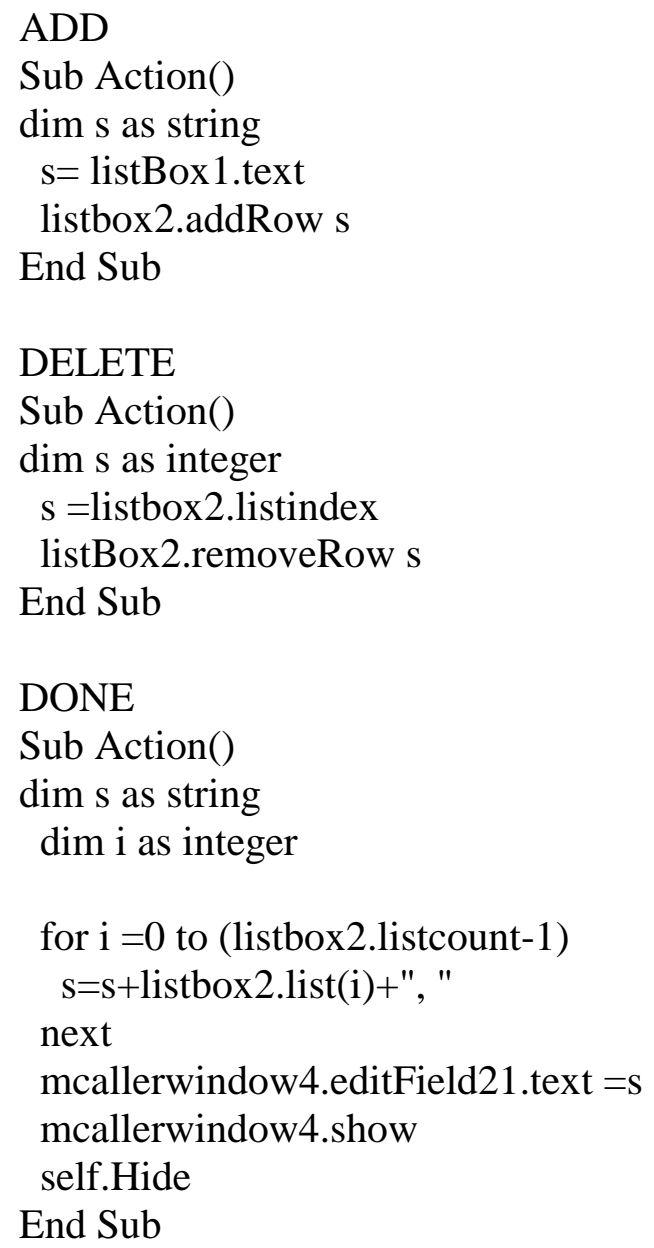


CANCEL

Sub Action()

self.hide

mcallerwindow4.show

EndSub

\section{Target_DATABASE:}

\section{Properties}

Mcallerwindow5 as Produicts_Database1

\section{Controls}

ADD

DELETE

DONE

CANCEL

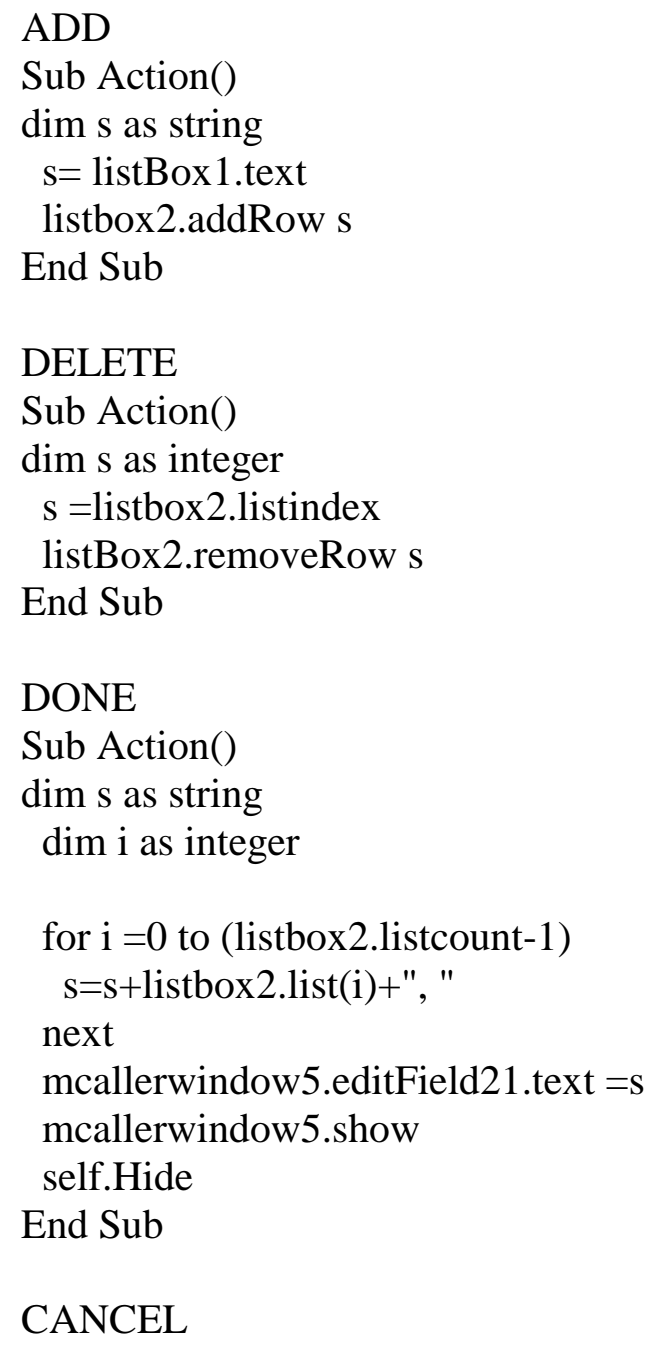

CANCEL 
Sub Action()

self.hide

mcallerwindow5.show

EndSub

\section{QueryWindow:}

\section{Properties}

mcallerwindow as produCTS_DATABASE1

mcursor as vcursor

\section{Methods}

Populatepanes

Sub populatepanes()

'results_DATABASE.show

'statictext2. Text $=$ Str ( mCursor. RecordCount $)$

TotalRecs. Text $=$ str(mCursor.RecordCount)

Editfield24.Text $=$ Str ( mCursor.CurrentPosition $)$

'results_DATABASE.mCursor2=self.mcursor

if $(\mathrm{mCursor}$.RecordCount $=0)$ then

'ClearPanes

return

end if

// we check if the field exists in the cursor, because

// in a query can be selected any fields

if( mCursor.Field("PRODUCT_\#") <> nil ) then

editField1.text $=$ mCursor.Field("PRODUCT_\#").GetString

end if

if( mCursor.Field("SF_\#") <> nil ) then

editField2.text $=$ mCursor.Field("SF_\#").GetString

end if

if( mCursor.Field("DW_\#") $<>$ nil ) then editField3.text $=$ mCursor.Field("DW_\#").GetString

end if

if( mCursor.Field("NETCSC_\#") $<>$ nil ) then editField4.text $=$ mCursor.Field("NETCSC_\#").GetString end if 
if( mCursor.Field("EPADoc_\#") $<>$ nil ) then editField7.text $=$ mCursor.Field("EPADoc_\#").GetString end if

if(mcursor.field("Year")<>nil) then editField8.text = mcursor.field("Year").GetString end if

if( mCursor.Field("Updated") $<>$ nil ) then editField9.text =mCursor.Field("Updated").GetString end if

if( mCursor.Field("Pages") $<>$ nil ) then editField10.text =mCursor.Field("Pages").GetString end if

if( mCursor.Field("Title") <> nil ) then editField11.text =mCursor.Field("Title").GetString end if

if( mCursor.Field("Price") <> nil ) then editField12.text =mCursor.Field("Price").GetString end if

if(mcursor.field("Stage") $<>$ nil ) then editfield22.text $=$ mcursor.field("Stage").GetString end if

if(mcursor.field("DateStart") $<>$ nil ) then editfield23.text $=$ mcursor.field("DateStart").GetString end if

if( mCursor.Field("Author") $<>$ nil ) then editField13.text $=$ mCursor.Field("Author").GetString end if

if( mCursor.Field("Affiliation") $<>$ nil ) then editField14.text $=$ mCursor.Field("Affiliation").GetString end if

if( mCursor.Field("Medium") $<>$ nil ) then editField15.text $=$ mCursor.Field("Medium").GetString end if

if( mCursor.Field("Reviewers") <> nil ) then 


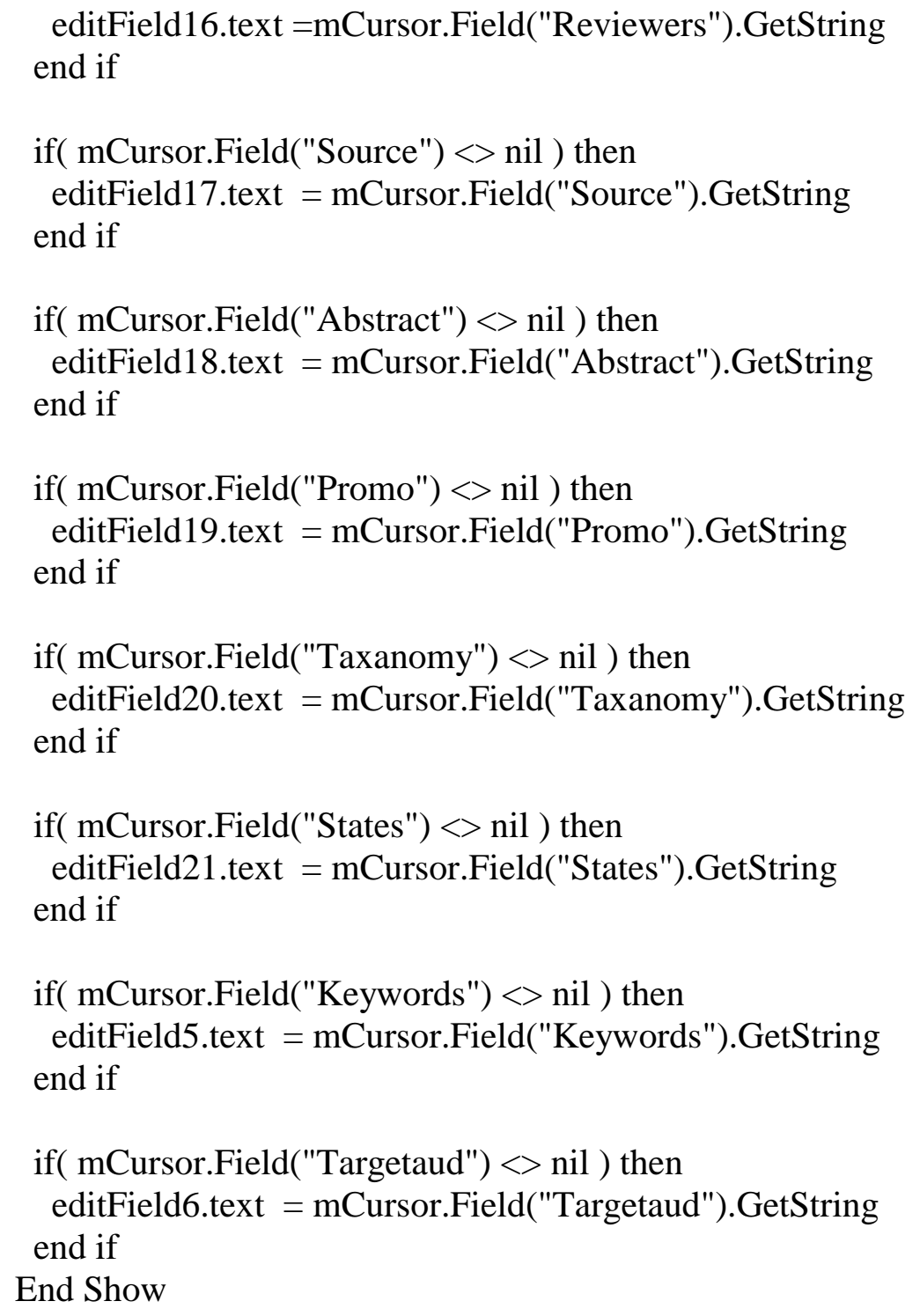

\section{Controls}

Submit

View

Cancel

FIRST

PREV

NEXT

LAST

RETURN 


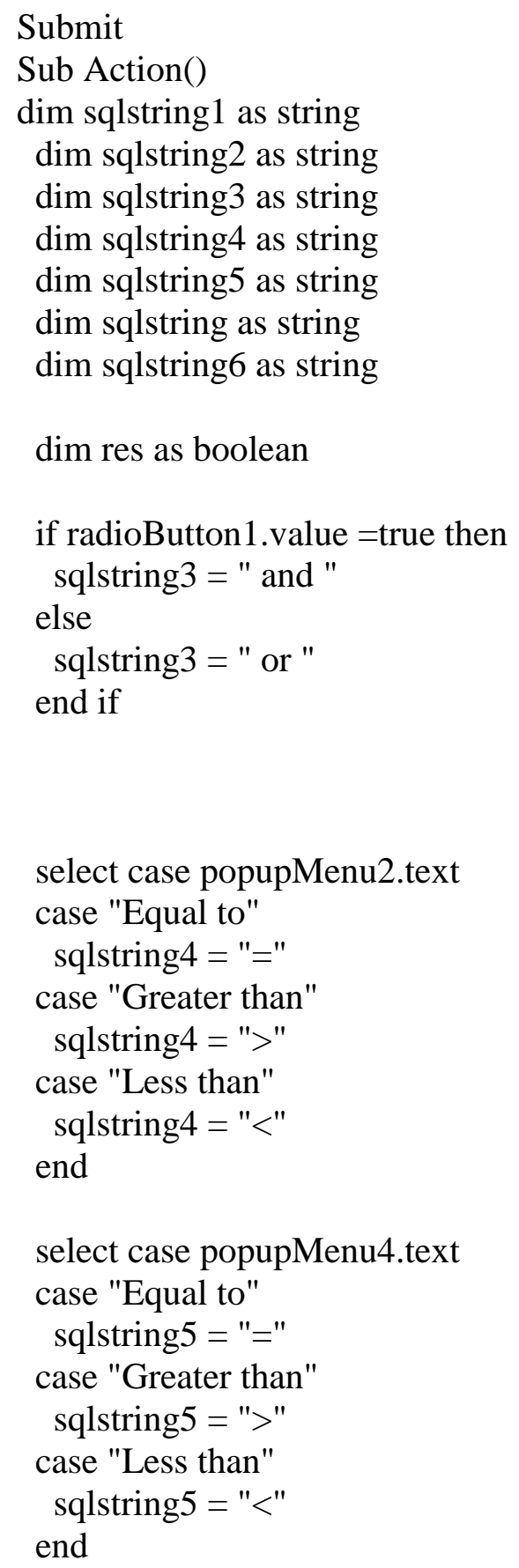


end if

if ( checkbox2.value $=$ true and checkBox1.value $=$ false) then sqlstring $=$ sqlstring 6

end if

if $($ checkbox2.value $=$ true and checkBox 1 .value $=$ true $)$ then

if (radioButton1.value $=$ false and radioButton2.value $=$ false $)$ then msgbox"choose either AND or OR"

elseif (radioButton1.value $=$ true or radioButton2.value $=$ true) then sqlstring=sqlstring1+sqlstring3+sqlstring2

end if

end if

if $($ checkbox2.value $=$ false and checkBox1.value $=$ false $)$ then msgbox"please check atleast one checkBox"

sqlstring $=" "$

end if

mCursor=nil

'ClearPanes

mCursor $=$ mCallerWindow.mDatabase.SQLselect(sqlstring)

if $\mathrm{mCursor}\langle>$ nil then

res $=$ mcursor.firstRecord

mCursor. CurrentPosition $=1$

'PopulatePanes

end if

recordnumber.text $=\mathrm{str}$ (mCursor.recordCount)

if $\mathrm{mCursor}=$ nil then

res =mcursor.firstRecord

'populatePanes

end if

End Sub

View

Sub Action()

'querywindow.title $=$ Results Window

//Hide Query related buttons

popupMenu1.visible $=$ false

popupmenu2. visible $=$ false

popupMenu3. visible $=$ false

popupmenu 4 . visible $=$ false

Submit.visible $=$ false

view. visible $=$ false 


$$
\begin{aligned}
& \text { cancel.visible }=\text { false } \\
& \text { string1.visible }=\text { false } \\
& \text { string2. } \text { visible }=\text { false } \\
& \text { radioButton } 1 . \text { visible }=\text { false } \\
& \text { radioButton } 2 . \text { VISible=FAlse } \\
& \text { checkBox } 1 . \text { visible }=\text { false } \\
& \text { checkBox } 2 . \text { visible }=\text { false } \\
& \text { recordtextt.visible=false } \\
& \text { recordnumber.visible=false } \\
& \text { //Show Results related fields }
\end{aligned}
$$

editfield 1. visible $=$ true editfield2. visible $=$ true editfield3. visible $=$ true editfield4 . visible $=$ true editfield5. visible $=$ true editfield6. visible $=$ true editfield 7 . visible $=$ true editfield8. visible $=$ true editfield9. visible $=$ true editfield10. visible $=$ true editfield11. visible $=$ true editfield12. visible $=$ true editfield13. visible $=$ true editfield14.visible $=$ true editfield15. visible $=$ true editfield16. visible $=$ true editfield17. visible $=$ true editfield18. visible $=$ true editfield19. visible $=$ true editfield20. visible $=$ true editfield21.visible $=$ true editfield22. visible $=$ true editfield23. visible $=$ true editfield24. visible $=$ true TotalRecs. visible $=$ true

StaticText1. visible $=$ true staticText2. visible $=$ true StaticText3 .visible $=$ true StaticText4. visible $=$ true StaticText5.visible $=$ true StaticText6. visible $=$ true StaticText7. visible $=$ true StaticText8. visible $=$ true Static Text9. visible $=$ true 


\begin{abstract}
StaticText10. visible $=$ true
StaticText11. visible $=$ true StaticText12. visible $=$ true StaticText13. visible $=$ true StaticText14. visible $=$ true StaticText15.visible $=$ true StaticText16.visible $=$ true StaticText17.visible $=$ true StaticText18.visible $=$ true StaticText19. visible $=$ true StaticText20.visible $=$ true StaticText21.visible $=$ true StaticText22.visible $=$ true StaticText23. visible $=$ true StaticText24. visible $=$ true StaticText25.visible $=$ true

bevelButton4 . visible $=$ true bevelButton5. visible $=$ true bevelButton6. visible $=$ true bevelButton 7. visible $=$ true return_b.visible=true populatepanes
\end{abstract}

\title{
End Sub
}

Cancel

Sub Action()

self.hide

mcallerwindow.show

\section{End Sub}

\section{FIRST}

Sub Action()

dim res as boolean res $=$ mcursor.firstRecord populatePanes

End Sub

\section{PREV}

Sub Action()

dim res as boolean if(mcursor.currentPosition $>1$ ) then res =mcursor.prevRecord populatePanes 
end if

End Sub

\section{NEXT}

Sub Action()

dim res as boolean

if (mcursor.currentposition < mcursor.recordCount)then res $=$ mcursor.nextRecord

populatePanes

end if

\section{End Sub}

\section{LAST}

Sub Action()

dim res as boolean

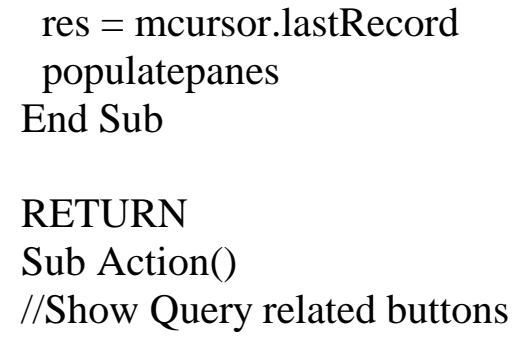

\section{RETURN}

Sub Action()

//Show Query related buttons

popupMenu1 .visible=true popupmenu2. visible $=$ true popupMenu3. visible $=$ true popupmenu4.visible $=$ true Submit.visible $=$ true view visible $=$ true cancel.visible $=$ true string1 visible $=$ true string2. visible $=$ true radioButton 1 . visible $=$ true radioButton2. VISible $=$ true checkBox1.visible $=$ true checkBox2.visible $=$ true recordtextt. visible $=$ true recordnumber. visible $=$ true

//Hide Results related fields

editfield 1 . visible $=$ false editfield2. visible $=$ false editfield3. visible $=$ false 


$$
\begin{aligned}
& \text { editfield4.visible }=\text { false } \\
& \text { editfield5.visible }=\text { false } \\
& \text { editfield6.visible }=\text { false } \\
& \text { editfield7.visible }=\text { false } \\
& \text { editfield8. } v \text { isible }=\text { false } \\
& \text { editfield9.visible }=\text { false } \\
& \text { editfield10.visible }=\text { false } \\
& \text { editfield11.visible }=\text { false } \\
& \text { editfield12.visible }=\text { false } \\
& \text { editfield13.visible }=\text { false } \\
& \text { editfield14.visible }=\text { false } \\
& \text { editfield15.visible }=\text { false } \\
& \text { editfield16.visible }=\text { false } \\
& \text { editfield17.visible }=\text { false } \\
& \text { editfield18.visible }=\text { false } \\
& \text { editfield19.visible }=\text { false } \\
& \text { editfield20.visible }=\text { false } \\
& \text { editfield21.visible }=\text { false } \\
& \text { editfield22.visible }=\text { false } \\
& \text { editfield23.visible }=\text { false } \\
& \text { editfield24.visible }=\text { false } \\
& \text { TotalRecs. } \text { visible }=\text { false }
\end{aligned}
$$

StaticText1. visible $=$ false staticText 2 . visible $=$ false StaticText3. visible $=$ false StaticText4. visible $=$ false StaticText5.visible $=$ false StaticText6. visible $=$ false StaticText7. visible $=$ false StaticText8. visible $=$ false StaticText9. visible $=$ false StaticText10.visible $=$ false StaticText11.visible $=$ false StaticText12. visible $=$ false StaticText13. visible $=$ false StaticText14. visible $=$ false StaticText15. visible $=$ false StaticText16.visible $=$ false StaticText17. visible $=$ false StaticText18.visible $=$ false StaticText19. visible $=$ false StaticText20.visible $=$ false StaticText21. visible $=$ false StaticText22. visible $=$ false StaticText23. visible $=$ false 


\begin{abstract}
StaticText24. visible $=$ false
StaticText25. visible $=$ false

bevelButton4. visible $=$ false

bevelButton 5 . visible $=$ false

bevelButton6. visible $=$ false

bevelButton 7. visible $=$ false

return_b.visible $=$ false
\end{abstract}

End Sub

\title{
Confirmation:
}

\section{Properties}

Mcallerwindow as Products_Database1

\section{Controls}

$\mathrm{OK}$

CANCEL

$\mathrm{OK}$

Sub Action()

dim res as boolean

res =mcallerwindow4.mdatabase.mfrontend.deleteRecord

mcallerwindow4.populatedb

self.hide

mcallerwindow4.show

End Sub

CANCEL

Sub Action()

self.hide

mcallerwindow4.show

End Sub

\section{Events}

Open

Sub Open()

statictext1.text = "Are you sure you want to delete the record ?"

End Sub 